July 2019

"Nonparametric Assessment of Hedge Fund Performance"

Caio Almeida, Kym Ardison and René Garcia 


\title{
Nonparametric Assessment of Hedge Fund Performance
}

\author{
Caio Almeida *Kym Ardison ${ }^{\dagger}$ René Garcia ${ }^{\ddagger}$
}

May 31, 2019

\begin{abstract}
We propose a new class of performance measures for Hedge Fund (HF) returns based on a family of empirically identifiable stochastic discount factors (SDFs). The SDF-based measures incorporate no-arbitrage pricing restrictions and naturally embed information about higher-order mixed moments between HF and benchmark factors returns. We provide a full asymptotic theory for our SDF estimators to test for the statistical significance of each fund's performance and for the relevance of individual benchmark factors within each proposed measure. We apply our methodology to a panel of 4815 individual hedge funds. Our empirical analysis reveals that fewer funds have a statistically significant positive alpha compared to the Jensen's alpha obtained by the traditional linear regression approach. Moreover, the funds' rankings vary considerably between the two approaches. Performance also varies between the members of our family because of a different fund exposure to higherorder moments of the benchmark factors, highlighting the potential heterogeneity across investors in evaluating performance.
\end{abstract}

Keywords: Hedge Funds, Admissible Performance Measures, Nonparametric Estimation, Higher-order Moments.

JEL Code: G12, G13, C14, C58

*Email: calmeida@fgv.br, EPGE/FGV and Princeton University, Princeton, USA.

$\dagger$ Email: kym.ardison@fgvmail.br, EPGE/FGV, Rio de Janeiro, Brazil.

‡Email: rene.garcia@umontreal.ca, Université de Montréal, Canada, and Toulouse School of Economics, France. 


\section{Introduction}

Investment management and portfolio performance evaluation go hand in hand. Professional portfolio managers propose services to investors in the form of funds. Pension committees, endowments or retail investors need to assess whether the proposed funds achieve a superior performance to the portfolios they could form given their available information. Investors will seek funds that deliver a superior performance achieved with dynamic strategies built on a refined information set.

To evaluate performance, investors rely mainly on a series of ratios (Sharpe, Information, Sortino, Treynor, Omega to name the main ones) or on a Jensen's alpha with respect to a set of benchmark assets. In a seminal paper, Chen and Knez (1996) propose a general framework for evaluating the performance of a managed portfolio with respect to a set of benchmarks and specify a set of conditions for a performance measure to be admissible. They introduce a class of positive admissible performance measures $\alpha($.$) that$ can be represented by a linear functional $\alpha\left(R_{i}^{H F}\right)=E\left[m R_{i}^{H F}-1\right]$, with $m>0$ being a strictly positive admissible SDF. These conditions exclude the Jensen's alpha measure but still accept a large set of positive admissible measures that guarantee no-arbitrage when evaluating managed returns. However, their empirical analysis, focused in analyzing a subset of mutual fund returns, concentrates only on two versions of the Hansen and Jagannathan (1991) SDF, the unconstrained and the non-negatively constrained ones. The performance measures implied by these two SDFs are both admissible but not always positive. Positivity (or not) of these measures directly depends on the particular dataset of observed benchmark returns.

In this paper, we aim at finding a family of admissible positive measures that are particularly suited to evaluate the performance of complex dynamic strategies such as the ones proposed by hedge funds. Performance evaluation for such funds has been mainly conducted with linear factor models that include the index returns of main asset classes and the returns on derivative positions with respect to these benchmarks (Fung and Hsieh (2001) and Agarwal and Naik (2004) are two prominent examples of such an approach). This approach recognizes that hedge funds can short assets and use derivatives to manage their portfolios, creating highly nonlinear payoffs. However, the performance measure is not guaranteed to be positive, and positivity is fundamental to rank managed 
funds consistently. Moreover, the linear exposure to the derivative factor payoffs does not capture the concave relations between the individual hedge fund strategy and the derivative benchmark returns since the inherent strike positions of the latter usually differ from the fund position ${ }^{1}$.

We provide a broad set of strictly positive SDFs, all sensitive to higher moments of the joint distribution of the benchmark assets and each generating a positive admissible performance measure. The performance is shown to depend on all the co-moments of the benchmark returns with each hedge fund returns, generalizing the linear factor models that include co-skewness (Harvey and Siddique, 2000) and co-kurtosis (Dittmar, 2002) with the market returns.

We minimize general convex functions of stochastic discount factors (SDF) called Minimum Discrepancy (MD) measures (Corcoran, 1998) in order to obtain a projected nonlinear SDF that prices exactly a set of selected reference assets (Almeida and Garcia, 2017). A well-known example of such discrepancy measures is the Kullback-Leibler information criteria (KLIC) or entropy ${ }^{2}$. Our family of discrepancy functions offers other information criteria that have different implications for assessing performance.

The solutions for the nonlinear SDFs are obtained through dual portfolio problems that are easier to solve than the primal problems. This extends the duality between the minimum-variance SDF and the quadratic portfolio problem in Hansen and Jagannathan (1991). The first-order conditions for these portfolio optimization problems provide solutions for the optimal weights of the chosen reference assets. We provide a complete estimation and inference theory for these weights and the resulting performance alpha, which measures the expected discounted value of the fund return under the nonlinear SDF. Given the small number of returns often found in individual hedge fund returns, we complement these asymptotic tests with bootstrap tests.

From an investor perspective, our measures will give more weight to positive returns in states of nature where marginal utility is high (bad times), a feature that traditional performance measures fail to capture (Kosowski, 2011). An important element is that

\footnotetext{
${ }^{1}$ de Roon and Karehnke (2017) construct a put-writing strategy as in (Jurek and Stafford, 2015) and show that a linear regression of this put on the market-excess returns is unable to account for the concave, nonlinear relation between the put and the index.

${ }^{2}$ Stutzer (1996) used the KLIC entropy measure to extract risk-neutral probabilities to price a set of options. Bansal and Lehmann (1997), Backus et al. (2011) and Backus et al. (2014) adopted the Empirical Likelihood entropy as a measure of dispersion to compare asset pricing models.
} 
each measure will put different weights on the states of nature and in this sense can be assimilated to an investor with a particular attitude towards risk. However, our approach differs from the utility-based evaluation of performance since it is nonparametric and based on pricing exactly the benchmark risk factors. In this paper, we emphasize the potential divergence between investors in evaluating a particular fund. This contrasts with the usual performance evaluation with ratios or with the Jensen's alpha where all investors share the same criterion. However, we keep the same economic interpretation of the alpha as the difference between an active portfolio and a passive one invested in benchmark assets, except that the active portfolio is chosen with different nonlinear objective functions.

The implementation of the performance measurement tests involves the selection of the benchmark factors, also called reference or basis assets. We choose four sets of reference assets that have been used in the literature on alpha measurement in hedge funds. The most popular set is the ten-factor model from Fung and Hsieh (2001), where risk factors including equities, bonds, credit, currencies, and commodities are considered together with several trend following strategies. We also include in our analysis a ninefactor model where we replace the Fung and Hsieh (2001) trend following strategies by the options strategies from Agarwal and Naik (2004). The Carhart (1997) four-factor model (the three Fama and French (1993) factors plus the momentum factor), and the CRSP value-weighted market portfolio are added for comparison purposes and completeness.

We evaluate the performance of hedge funds reporting to the Lipper-Tass database. It is well-known that commercial hedge fund databases suffer from severe biases (e.g. Fung and Hsieh (2001), Aggarwal and Jorion (2010), Patton et al. (2015), Bollen and Pool (2009) and Aiken et al. (2013) among several others). Traditionally, the literature focuses on two primary sources of bias: survivorship and backfill (e.g. Fung and Hsieh (2001) and Aggarwal and Jorion (2010) respectively). Recent articles have raised some additional issues. Straumann (2009) notes that several commercial databases (including the one we used in this paper) suffer from serious problems such as an alarming number of hedge funds with excessive zero returns, consecutive equal returns, and repeated "blocks" of returns ${ }^{3}$. To conduct our evaluation of performance, we apply the strictest filters that have been put forward to eliminate patterns that could bias our findings.

\footnotetext{
${ }^{3}$ Bollen and Pool (2012) investigated these suspicious patterns in returns and showed that several of them are indicative of a heightened risk of fraud.
} 
In our empirical analysis, we will contrast our approach with the Jensen's alpha as well as the Hansen and Jagannathan (1991) measure used in Chen and Knez (1996). Our family of measures will be indexed by a parameter $\gamma$ that will capture the curvature of the functions optimized in our dual portfolio problems. A Taylor expansion of our performance measure shows the intrinsic relationship between the fund's performance and its sensitivity to the higher-order mixed moments with respect to the benchmark assets. In particular, depending on the value of $\gamma$ more or less weight is given to, say, co-skewness and co-kurtosis. For values close to one (Hansen and Jagannathan case) the weights assigned to higher-order moments are negligible, as in the case of the Jensen's alpha. On the contrary, moving to the negative values $(\gamma<0)$, positive weights are assigned to co-skewness (consistent with Kraus and Litzenberger (1976) skewness preference) and negative weights are assigned to co-kurtosis (kurtosis aversion, Dittmar (2002)). In the latter two papers, the co-skewness and co-kurtosis statistics are computed with respect to the market returns, while our method is neither restricted to a single asset nor does it rely on imprecise estimates of higher-order co-moments.

As we mentioned, we want to put forward how different investors (indexed by the parameter $\gamma$ ) will evaluate the performance of a fund. It raises the issue of how a particular investor will know which $\gamma$ will correspond to his own risk preferences. We provide in Section 6.3.1.6 a possible procedure to be followed based on the current portfolio of the investor. We also indicate how much of this hedge fund should be added to the current portfolio.

Our analysis puts forward several important empirical findings. First, we consider the statistical significance of the selected reference assets. A reassuring fact is that all investors ${ }^{4}$ will want to hold a statistically significant positive share of the market, regardless of the set of factors we consider. With the Carhart (1997) set of factors, both the high-minus-low and the momentum factors are significant and all investors are long in these two factors in the dual portfolio. When we consider the Fung and Hsieh (2001) set of factors, the credit spread appears as an additional significant asset held positively in all portfolios, while the only significant trend following strategy is to short the stock lookback straddle. The results for the Agarwal and Naik (2004) model, where we switch the trendfollowing factors for the option portfolios, are somewhat consistent. The weights assigned

\footnotetext{
${ }^{4}$ We use a slight abuse of language by assimilating an investor to a particular values of $\gamma$ in our family of measures.
} 
to the S\&P 500 index returns increase substantially when the options are introduced in the estimation of the dual portfolios, and remarkably all investors short out-of-the-money puts and buy the at-the-money ones.

Next, we compute the alpha performance measures for the categories of strategies used in the Lipper-Tass data base to group the individual hedge funds based on their own reporting. The first conclusion is that the estimated Jensen's alpha and HansenJagannathan alpha (based on a non-negative linear SDF that prices exactly the reference assets) are practically identical for all categories of hedge funds. More notable differences appear for the other values of the curvature parameter $\gamma$. For several categories such as CTA, fixed-income arbitrage, global macro, managed futures and funds of funds, the estimate for very negative values of $\gamma$ is significantly lower than in the linear case ${ }^{5}$ These important differences at the aggregate level of fund categories are indicative of a strong heterogeneity in performance at the individual fund level. In terms of cross-sectional distributions, the estimated alphas differ considerably between the $\gamma \mathrm{s}$ and across the sets of reference assets. We also show that the ranking of the funds differs considerably between the Jensen's alpha measure and our new proposed measures putting more weight on bad states of the world (see Figure 10).

We have already connected our paper to several strands of the literature on performance evaluation and on hedge-fund data biases. We conclude this introduction by mentioning several papers on the performance evaluation of hedge funds and how we do relate to them. Kosowski et al. (2007) use a robust bootstrap procedure to evaluate the performance of hedge funds. The bootstrap ensures that the performance measure is not produced by luck and accounts, together with the Bayesian estimation method, for the short-sample problem inherent in hedge fund returns. We also double our asymptotic tests with bootstrap tests that are robust when testing for the significance of the hedge funds alphas. Capocci and Hubner (2004) investigate the performance of hedge funds using various asset pricing models, including the Carhart (1997) and the Agarwal and Naik (2004) models. We include these sets of factors in our analysis and extend their linear specification to our family of nonlinear positive SDFs. Hubner et al. (2015) put forward the key roles of US equity skewness and kurtosis in the hedge fund return generating process. We show that the cross-section of hedge fund alphas indeed strongly depends on

\footnotetext{
${ }^{5}$ We refer here to the results based on the Agarwal and Naik benchmark assets (see Table A11).
} 
the co-skewness and co-kurtosis with the market returns. Goetzmann et al. (2007) characterize manipulation-proof performance measures showing that, in general, they should be similar to a power utility function averaged across time. Billio et al. (2013) extend their work proposing utility-based measures that consider moments up to the fourth-order to measure performance. Our nonparametric approach also provides manipulation-proof performance measures, does not depend on specifying utility models, and endogenously captures all moments of the joint distribution of the benchmark factors.

Our paper is also intimately related to the SDF performance measurement literature (e.g. Beja (1971)) . More specifically, Farnsworth et al. (2002), Ferson and Mo (2016) and Li et al. (2016) propose several SDF-based performance measures. As already mentioned, our approach extends the traditional linear SDF approach in Hansen and Jagannathan (1991) by providing a continuum of positive measures with different degrees of nonlinearities. As in Ferson and Mo (2016), investors with different attitudes towards risk evaluate performance differently. However, our non-parametric SDFs have zero pricing errors with respect to the benchmark assets.

The rest of the paper is organized as follows. In Section 2, we describe the theoretical properties of our performance measures while section 3 offers an interpretation and comparison of these measures with previously proposed alpha estimators. Section 4 describes our estimation method and the asymptotic properties of our estimators. Section 5 gives a detailed account of the data set and the multiple filters we apply to the data to construct reliable measures of performance. Section 6 contains all our empirical findings. First, we describe the estimated measures for the various sets of reference assets. Then we report the performance measures and their asymptotic and bootstrap t-statistics both for aggregate categories of funds and individual funds. Section 7 concludes. For space considerations, we have placed all theorem and lemma proofs as well as many tables and figures (with numbers preceded by an A) in an online Appendix.

\section{A Family of Nonparametric Performance Measures}

Let us first define the probabilistic and economic environments in which we are going to analyze the performance of hedge fund investments. Let $(\Omega, \mathcal{F}, P)$ denote a probability

\footnotetext{
${ }^{6}$ For a careful review of this literature see Ferson (2013).
} 
space, where $\Omega$ is the set of states of nature, $\mathcal{F}$ a sigma-field on $\Omega$, and $P$ a probability measure. Let $R^{K}=\left\{R_{1}, R_{2}, \ldots, R_{K}\right\}$ represent a set of square-integrable benchmark returns, i.e, for $\forall i R_{i} \in L^{2}(\Omega)$, with $L^{2}(\Omega)=\left\{X: \Omega \rightarrow \mathbb{R}: E\left(X^{2}\right)<\infty\right\}^{7}$. Denote by $S^{s}=\left\{R \in L^{2}(\Omega): \exists s \in S, \theta(s) \in \mathbb{R}^{K}\right.$ s.t. $\sum_{k \in K} \theta_{k}(s)=1$ and $\left.\sum_{k \in K} \theta_{k}(s) R_{k}=R\right\}$ the set of all achievable managed returns, where $S$ denotes the collection of all private signals available to investment managers, i.e. random variables containing information about the benchmark assets that can be used to generate a rich set of trading strategies. Note that for each private signal $s, \theta(s)$ is a normalized random vector representing a trading strategy that is a function of $s$. We denote by $S^{p}$ the set of investments available to the general public. $S^{p}$ is the subset of $S^{s}$ where $\theta(s)$ does not depend on $s$, meaning that the general public does not have access to private signals. In this context, a manager's non-null performance is achieved via exploring the private nature of signals to generate complex payoffs that do not lie on the span of the publicly available investments $S^{p}$. Throughout the paper, $\alpha: S^{s} \rightarrow \Re$ will denote a generic performance measurement, $R_{i}^{H F}$ the return of an individual hedge fund $i, R_{k}$ the return of the $k$ th benchmark portfolio, with $k \in\{1,2, \ldots, K\}$, and $\mathbf{R}=\left(R_{1}, R_{2}, \ldots, R_{K}\right)^{\prime}$ the vector of benchmark returns.

In a seminal paper, Chen and Knez (1996) introduce an important class of positive admissible performance measures. They prove that each of these measures $\alpha($.$) can$ be represented by a linear functional $\alpha\left(R_{i}^{H F}\right)=E\left[m R_{i}^{H F}-1\right]$, with $m>0$ being a strictly positive admissible $\mathrm{SDF}^{8}$. Each of these performance measures satisfies a set of fundamental conditions that guarantees consistency in ranking any set of managed returns. First, and most importantly, if a managed return, $R_{i}^{H F}$, lies in the affine hull of the set of basis assets $R^{K}$ (i.e., $R_{i}^{H F} \in S^{p}$ ), then it should receive zero performance, $\alpha\left(R_{i}^{H F}\right)=0$. In other words, passive portfolio management leads to zero performance. In addition, positive admissible performance measures are: linear functions of returns, such that no return rearranging by a manager can improve performance $\left(\alpha\left(\beta_{1} R_{i}^{H F}+\right.\right.$ $\left.\left.\beta_{2} R_{j}^{H F}\right)=\beta_{1} \alpha\left(R_{i}^{H F}\right)+\beta_{2} \alpha\left(R_{j}^{H F}\right)\right)$; continuous in the sense that managed returns that are similar should receive a similar performance value; nontrivial, i.e., it is not the case that

\footnotetext{
${ }^{7}$ Benchmark returns could be returns associated with indexes of stocks, bonds, credit securities, currencies, as well as returns on options based on these indexes. See Fung and Hsieh (2001) and Agarwal and Naik (2004) for concrete examples of sets of benchmarks adopted in the hedge fund performance literature.

${ }^{8} \mathrm{An}$ admissible SDF is a random variable $m$ that prices all benchmark assets: $E\left[m R_{k}\right]=1, R_{k} \in R^{K}$.
} 
$\alpha\left(R_{i}^{H F}\right)=0$ for all $R_{i}^{H F} \in S^{s}$; and strictly positive, i.e. $\alpha\left(R_{i}^{H F}\right)>0$ whenever $R_{i}^{H F}>0 .{ }^{9}$ Strict positivity is a fundamental condition to guarantee ranking consistency in the sense that if a managed return $R_{1}^{H F}$ dominates a managed return $R_{2}^{H F}\left(R_{1}^{H F}>R_{2}^{H F}\right)$, then $\alpha\left(R_{1}^{H F}\right)>\alpha\left(R_{2}^{H F}\right)^{10}$.

Assuming a structure of arbitrage-free incomplete markets where there exists an infinity of SDFs, Chen and Knez (1996) provide theoretical foundations for positive admissible performance measures as well as some methodological guidance for future development of such measures. Nonetheless, in empirical applications they fail to exhibit positive admissible measures. Their empirical analysis, focused in analyzing a subset of mutual fund returns, concentrates only on two versions of the Hansen and Jagannathan SDF, the unconstrained and the non-negatively constrained ones. The performance measures implied by these two SDFs are both admissible but not always positive. Positivity (or not) of these measures directly depends on the particular dataset of observed benchmark returns. In fact, depending on the realized history of benchmark returns, the non-negatively constrained implied SDF could achieve zeros in some states of nature, while the unconstrained HJ SDF would achieve negative values in the corresponding states.

Figure 5 exhibits the non-negatively constrained HJ SDF implied by two typical sets of benchmark factors, one adopted by Fung and Hsieh (2001) (left bottom) and the other by Agarwal and Naik (2004) (right bottom). Note that for both datasets of benchmark factors the constrained HJ SDF achieves zeros in respectively five and two states out of a total of 250 states. This implies that, if we were analyzing hedge funds' performance based on the usual datasets of benchmark factors, both HJ performance measures could potentially rank managed returns inconsistently. Moreover, since the performance measure implied by the unconstrained Hansen and Jagannathan SDF is equivalent to the APT multi-factor version of the Jensen's alpha (Chen and Knez, 1996) adopted in the hedge fund literature, the Jensen's alpha itself would also potentially rank managed returns inconsistently. Therefore, it is important to propose alternative admissible measures to analyze hedge fund performance.

The examples above make clear that the most significant challenge is to search for

\footnotetext{
${ }^{9} R>0$ means that $R$ is non-negative and $P(R>0)>0$.

${ }^{10}$ It should be clear that Chen and Knez (1996) also define and analyze admissible performance measures that can be represented by $\alpha\left(R_{i}^{H F}\right)=E\left[m R_{i}^{H F}-1\right]$, with $m$ being any admissible SDF, not necessarily positive. We are not particularly interested in those measures since they do not provide a consistent ranking of funds.
} 
ways to identify strictly positive admissible SDFs $(m)$ extracted from empirical data on benchmark assets. Our main contribution is to propose a methodology to identify such a family of strictly positive SDFs. We provide a broad set of strictly positive SDFs, all sensitive to higher moments of the joint distribution of the benchmark assets and each generating a positive admissible performance measure.

We now formally introduce our family of performance measures. We are looking for admissible risk-adjustment weights, also known as stochastic discount factors, that make the average weighted excess returns on the factors equal to zero: ${ }^{11}$

$$
E\left[m\left(\mathbf{R}-1_{K}\right)\right]=0_{K}
$$

where $0_{K}$ is a $\mathrm{K}$-dimensional vector of zeros and $\mathbf{R}$ the $\mathrm{K}$-dimensional vector of benchmark returns.

In an incomplete market setting ${ }^{12}$ where the law of one price is satisfied, there are many such weighting functions (see (Cochrane, 2001)). Therefore, we need to restrict the set of such admissible SDFs. Hansen and Jagannathan (1991) find an admissible linear SDF with minimum variance, obtained by minimizing a quadratic function in the space of admissible SDFs. Instead, assuming absence of arbitrage on the market defined by the benchmark assets (primitive risk-factors) ${ }^{13}$, we consider a convex discrepancy function $\phi$ and we search for a Minimum Discrepancy (MD) SDF that solves the following minimization problem in the more restricted space of admissible strictly positive SDFs:

$$
m_{M D}=\underset{m}{\arg \min } E[\phi(m)]
$$

subject to $E\left[m\left(\mathbf{R}-1_{K}\right)\right]=0_{K}, E[m]=1, m>0$.

In this optimization problem, conditions $E\left[m\left(\mathbf{R}-1_{K}\right)\right]=0_{K}$ and $E[m]=1$ must

\footnotetext{
${ }^{11}$ We fix the SDF mean to one without loss of generality. If the SDF mean is not equal to one, the bounds are easily obtained with Equation (1) taking the following form: $E\left[m\left(\mathbf{R}-R_{F} 1_{K}\right)\right]=0_{K}$, where $R_{F}$ represents the risk-free rate.

${ }^{12}$ Going from the population to the sample problem, we have a $T \times K$ panel of benchmark data. In this context, market incompleteness emerges in any one-period model where the number of states $T$ is higher than the number of benchmark assets $K$. Since typically the number of benchmark factors does not exceed 15 while the minimum size of a time series of hedge fund returns analyzed in the literature is of 24 monthly observations, the problem of identifying an admissible SDF is usually set in an incomplete market environment.

${ }^{13}$ Absence of arbitrage on the market defined by the primitive risk-factors guarantees the existence of at least one admissible SDF that is strictly positive (see (Cochrane, 2001)).
} 
be obeyed by any admissible SDF $m$ with mean 1 . In addition, we explicitly impose a positivity constraint to guarantee that the implied MD SDF will generate a positive admissible performance measure. ${ }^{14}$

The sample version of the problem above is:

$$
\begin{aligned}
& \hat{m}_{M D}=\underset{\left\{m_{1}, \ldots m_{T}\right\}}{\arg \min } \frac{1}{T} \sum_{t=1}^{T} \phi\left(m_{t}\right), \\
& \text { subject to } \frac{1}{T} \sum_{t=1}^{T} m_{t}\left(\mathbf{R}_{t}-1_{K}\right)=0_{K}, \frac{1}{T} \sum_{t=1}^{T} m_{t}=1, m_{t}>0 \forall t,
\end{aligned}
$$

where $\mathbf{R}_{t}$ represents the realization of the vector of benchmark returns at time $t$.

The minimization, on its sample version, is based on the space of discrete strictly positive SDFs with dimension $T$, which can become impractical. Moreover, the corresponding problem in the population space (see Equation (2)) is an infinite dimensional optimization problem in the space of risk neutral probability measures. Building on Borwein and Lewis (1991), Almeida and Garcia (2017) show how the minimization problem in (2), and its corresponding sample version, can be solved in a generally much smaller finite-dimensional space of dimension $K$ by using the following dual problem:

$$
\lambda^{*}=\underset{\alpha \in \Re, \lambda \in \Lambda}{\arg \sup } \alpha-E\left[\phi^{*,+}\left(\alpha+\lambda^{\prime}\left(\mathbf{R}-1_{K}\right)\right)\right],
$$

whose corresponding sample version is given by:

$$
\hat{\lambda}=\underset{\alpha \in \Re, \lambda \in \Lambda}{\arg \sup } \alpha-\sum_{t=1}^{T} \frac{1}{T} \phi^{*++}\left(\alpha+\lambda^{\prime}\left(\mathbf{R}_{t}-1_{K}\right)\right)
$$

where $\Lambda \subseteq \Re^{K}$ and $\phi^{*,+}$ denotes the convex conjugate of $\phi$ restricted to the positive real line:

$$
\phi^{*+}(z)=\sup _{w>0} z w-\phi(w)
$$

Note that any convex discrepancy function can be chosen to arrive at empirical estimates of these minimum discrepancy SDFs. Following Almeida and Garcia (2017), we

\footnotetext{
${ }^{14}$ As shown by Almeida and Garcia (2017) and later in this section, the existence (or not) of a strictly positive solution to the MD problem will directly depend on the $\phi$ function chosen.
} 
adopt the Cressie-Read (1984) family of discrepancies defined as:

$$
\phi^{\gamma}(m)=\frac{(m)^{\gamma+1}-1}{\gamma(\gamma+1)}, \gamma \in \Re
$$

where each fixed value of $\gamma$ implies one specific discrepancy function. Almeida and Garcia (2017) show how to solve the dual problem and recover the admissible SDF from the optimization problem above. Here we synthesize their results in Corollary 1, which will be needed for us to develop the full asymptotic framework for our performance measures.

Corollary 1. Suppose we are interested in solving the minimization problem (2). Let $\phi(m)$ be as in (7), and assume that there is no-arbitrage in the economy, such that there exists at least one strictly positive admissible $\operatorname{SDF}^{15}$. Let $\Lambda(\mathbf{R})=\left\{\lambda \in \mathbb{R}^{K}\right.$ : $\left.\left(1+\gamma \lambda^{\prime}\left(\mathbf{R}-1_{K}\right)\right)>0\right\}$. Then, the dual problem (4) specializes to:

(i) if $\gamma>0$ :

$$
\lambda^{*}=\underset{\lambda \in \mathbb{R}^{K}}{\arg \sup } E\left[-\frac{1}{\gamma+1}\left(1+\gamma \lambda^{\prime}\left(\mathbf{R}-1_{K}\right)\right)^{\frac{\gamma+1}{\gamma}} I_{\Lambda(\mathbf{R})}(\lambda)\right]
$$

where $I_{A}(x)=1$ if $x \in A$ and 0 otherwise;

(ii) if $\gamma<0$ :

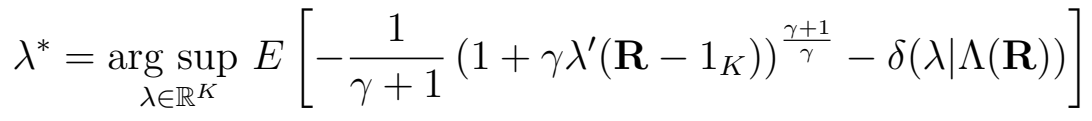

where $\delta(x \mid A)=0$ if $x \in A$ and $+\infty$ otherwise;

(iii) if $\gamma=0$, the following unconstrained problem:

$$
\lambda^{*}=\underset{\lambda \in \mathbb{R}^{K}}{\arg \sup } E\left[\exp \left(\lambda^{\prime}\left(\mathbf{R}-1_{K}\right)\right)\right] .
$$

Note that by solving the corresponding sample version of (8), (9) or (10) in Corollary 1, for each choice of $\gamma$ we obtain a distinct set of estimates for $\lambda\left(\hat{\lambda_{\gamma}}\right)$ that will lead to a different MD SDF $\left(\hat{m}^{\gamma}\right)$. The MD SDF $\hat{m}^{\gamma}$ is recovered by solving the first-order conditions of the problem and is known in closed form.

\footnotetext{
${ }^{15}$ To ease the notation we specify Almeida and Garcia (2017) corollary for the case where the SDF mean equals one.
} 
Corollary 2. Let $\hat{\lambda}_{\gamma}$ be the solution of the sample version of either (8), (9) or (10) in Corollary 1. Then, the corresponding MD implied SDF will be given by:

(i) if $\gamma>0$ :

$$
\hat{m}_{t}^{\gamma}=\left(1+\gamma \hat{\lambda}_{\gamma}^{\prime}\left(\mathbf{R}_{t}-1_{K}\right)\right)^{\frac{1}{\gamma}} I_{\Lambda\left(\mathbf{R}_{t}\right)}\left(\hat{\lambda}_{\gamma}\right)
$$

(ii) if $\gamma<0$ :

$$
\hat{m}_{t}^{\gamma}=\left(1+\gamma \hat{\lambda}_{\gamma}^{\prime}\left(\mathbf{R}_{t}-1_{K}\right)\right)^{\frac{1}{\gamma}}
$$

(iii) if $\gamma=0$ :

$$
\hat{m}_{t}^{\gamma}=\exp \left(\hat{\lambda}_{0}^{\prime}\left(\mathbf{R}_{t}-1_{K}\right)\right)
$$

for $t=1,2, \ldots, T$.

Since we search the Lagrange multipliers $\lambda$ in $\Lambda(\mathbf{R})=\left\{\lambda \in \mathbb{R}^{K}:\left(1+\gamma \lambda^{\prime}(\mathbf{R}-1)\right)>0\right\}$, for any $\gamma<0$ the implied MD SDF in (12) will be strictly positive. Moreover, for $\gamma=0$ the implied MD SDF is exponential, hence strictly positive too. Therefore, considering these two cases, we have already provided an infinite family of strictly positive SDFs (indexed by the continuous parameter $\gamma \leq 0$ ) that can be directly obtained from the time series of a set of benchmark returns by solving the dual problems in Corollary 1. Given each of these SDFs, a positive admissible performance measure is given by setting:

$$
\alpha^{\gamma}\left(R_{i}^{H F}\right)=E\left[m^{\gamma} R_{i}^{H F}-1\right]
$$

whose sample version is:

$$
\alpha^{\gamma}\left(R_{i}^{H F}\right)=\frac{1}{T} \sum_{t=1}^{T}\left[m_{t}^{\gamma} R_{i t}^{H F}-1\right] .
$$

Now, when $\gamma>0$, a case that includes the Hansen and Jagannathan SDF with nonnegativity constraint, we note that due to the indicator function $I_{\Lambda\left(\mathbf{R}_{t}\right)}\left(\hat{\lambda}_{\gamma}\right)$ that appears within solution (11), the implied MD SDF will achieve zeros in all the states of nature 
where $\left(1+\gamma \hat{\lambda}_{\gamma}^{\prime}(\mathbf{R}-1)\right) \leq 0$. As shown in Figure 5, typical benchmark datasets adopted to analyze hedge fund performance make the implied SDF for $\gamma=1$ (i.e, non-negatively constrained HJ SDF) to have zeros in some states. Therefore, for implied MD SDFs with $\gamma>0$, we can only guarantee to generate non-negative admissible performance measures. For some data sets these measures will be strictly positive, but not always. In the empirical section we analyze two performance measures in this sub-class of positive $\gamma$ 's, namely the cases with $\gamma=0.5$ and $\gamma=1$.

We have underlined the fact that there might exist multiple SDFs that correctly price the $K$ benchmark assets chosen to evaluate performance. Moreover, in the inherent incomplete-market setting that we described, the SDF might be investor dependent. That is, even though each investor prices the benchmark (traded) assets equally, the internal pricing structure might vary across investors (see Mas-Colell et al. (1995) for a formal treatment). In the hedge fund performance framework this means that if the hedge fund returns $R_{i}^{H F}$ are not spanned by the benchmark assets in the economy each investor might have a different fund valuation. In particular, let $m^{\gamma}$ be the SDF that corresponds to a particular investor with parameter $\gamma$. For this investor, the hedge fund performance is measured as $\alpha_{i}^{\gamma}\left(R_{i}^{H F}\right)=E\left[m^{\gamma} R_{i}^{H F}-1\right]$.

Note that, as mentioned before, if $R_{i}^{H F} \in S_{p}$, i.e. the hedge fund return is accessible to the general public trough an affine combination of the benchmark assets' returns, we have that $\alpha_{i}^{\gamma}\left(R_{i}^{H F}\right)=0 \forall \gamma \in \Re$. This is true since by construction any admissible SDF correctly prices all the benchmark assets and their corresponding affine combinations. Nonetheless, hedge funds might achieve superior information by making use of a private signal $s \in S$. This information can be used to construct state-dependent strategies such that $R_{i}^{H F}$ is not spanned by the benchmark assets. In this case, $\alpha^{\gamma}\left(R_{i}^{H F}\right) \neq 0$, and performance values will vary according to each investor $(\gamma)$, precisely as when we price unspanned payoffs (for instance, derivatives) in incomplete markets.

\section{Interpretation and Comparison of our Performance Measures}

In this section, we characterize the theoretical properties of the alphas obtained with various members of the Cressie Read family of SDFs and compare them with other 
available performance measures. We proceed with a Taylor expansion that links the performance measure with the higher-order mixed moments between the hedge fund return and the return of the optimal portfolio that solves the optimization on Corollary 1.

\subsection{Linking our Performance Measures with Higher-Order Mo- ments}

For a fixed $\gamma$ indexing the Cressie Read family of discrepancies, suppose that we solved the dual optimization problem on Corollary 1 . Let $\tilde{\mathbf{R}}=\mathbf{R}-1_{K}$, denote the vector of excess returns of benchmark assets over the risk-free rate $R_{F}$, here assumed to be constant and equal to 1 . Let also $W=-\lambda_{\gamma}^{\prime} \tilde{\mathbf{R}}$ represent the excess return of the optimal portfolio that solves the optimization on Corollary 1. Note that the specific problem (Equation (8), (9), or (10)) is determined by the value of $\gamma^{16}$.

Now, given the implied MD SDF $m^{\gamma}$ and the hedge fund return $R_{i}^{H F}$, we define the auxiliary function $g_{m, i}^{\gamma}$, which gives the risk-neutralized hedge fund return:

$$
g_{m, i}^{\gamma}\left(\lambda_{\gamma}^{\prime} \tilde{\mathbf{R}}\right)=\left(1+\gamma \lambda_{\gamma}^{\prime} \tilde{\mathbf{R}}\right)^{1 / \gamma} R_{i}^{H F}
$$

To see how the alpha performance $\alpha\left(R_{i}^{H F}\right)$ (in Equation 14) will vary with the parameter $\gamma$ and the co-moments of the benchmark returns with the hedge fund returns, we Taylor expand $g_{m, i}^{\gamma}\left(\lambda_{\gamma}^{\prime} \tilde{\mathbf{R}}\right)$ around $E[W]$, the expected excess return of the implied optimal portfolio. Expanding $g_{m, i}^{\gamma}($.$) and taking expectations we obtain:$

$$
\begin{aligned}
E\left[g_{m, i}^{\gamma}\left(\lambda_{\gamma}^{\prime} \tilde{\mathbf{R}}\right)\right] & =E\left[g_{m, i}^{\gamma}\left(E\left[\lambda_{\gamma}^{\prime} \tilde{\mathbf{R}}\right]\right)\right] \\
& -(1-\gamma E[W])^{\frac{1-\gamma}{\gamma}} E\left[R_{i}^{H F}(W-E[W])\right] \\
& +\frac{1}{2}(1-\gamma)(1-\gamma E[W])^{\frac{1-2 \gamma}{\gamma}} E\left[R_{i}^{H F}(W-E[W])^{2}\right] \\
& -\frac{1}{6}(1-\gamma)(1-2 \gamma)(1-\gamma E[W])^{\frac{1-3 \gamma}{\gamma}} E\left[R_{i}^{H F}(W-E[W])^{3}\right]+\ldots
\end{aligned}
$$

The above expansion reveals several noteworthy features. First, the alpha of a fund

\footnotetext{
${ }^{16}$ Almeida and Garcia (2012) and Almeida and Garcia (2017) showed how to link our nonparametric approach with the portfolio problem of an optimizing agent with HARA utility function. A key feature of our analysis here is that the portfolio holdings of the agent are proportional to $-\lambda$.
} 
is shown to explicitly depend on the relationship between its return and the higher-order mixed moments with the benchmark assets obtained via the optimal portfolio return $W$. In particular, the expansion indicates that the alpha is naturally linked to the co-skewness and co-kurtosis between the fund returns and the optimal portfolio return $W=-\lambda_{\gamma}^{\prime} \tilde{\mathbf{R}}$.

Ever since Kraus and Litzenberger (1976) a significant strand of the finance literature focused on understanding the implications of higher-order moments for asset pricing. In particular, Kimball (1993), and Dittmar (2002), among others, link investors' utility with their preferences for skewness and kurtosis. The all-important paper by Harvey and Siddique (2000) analyzes empirically the implications of systematic skewness and kurtosis for asset pricing ${ }^{17}$. In the hedge fund literature, Ranaldo and Favre (2003) show that higher-order moments are relevant for characterizing the returns of some funds on top of the market returns. In a related work, Billio et al. (2013) propose a new performance measure that takes into account the hedge fund skewness and kurtosis.

In contrast to this literature, our performance measure embeds information from all higher-order mixed moments with respect to the benchmark assets. In particular, two features are worth highlighting. First, our performance measure naturally embeds information about higher-order risks without estimating the risks themselves. This is a key feature since we avoid two main statistical problems - introducing too many parameters and facing the imprecise estimation of higher-order moments. Second, our measure contains information about higher-order risks related to a meaningful portfolio: the linear portfolio formed using all relevant benchmark assets. Note that the typical Harvey and Siddique (2000) approach becomes rapidly unfeasible in the hedge fund context since too many co-skewness and co-kurtosis statistics will have to be estimated for the numerous risk factors used to characterize returns ${ }^{18}$.

Further exploring each of the terms multiplying the mixed moments in (17) and noting that $E\left[g_{m, i}\left(E\left[\lambda_{\gamma}^{\prime} \tilde{\mathbf{R}}\right]\right)\right]$ can be rewritten as $\left(1+\gamma E\left[\lambda_{\gamma}^{\prime} \tilde{\mathbf{R}}\right]\right)^{\frac{1}{\gamma}} E\left[R_{i}^{H F}\right]$, we conclude that the hedge fund alpha is linearly related to its average return and that this average is re-scaled by the SDF evaluated at (minus) the mean value of the endogenous portfolio return. From the Jensen's inequality applied to the function $(\cdot)^{\frac{1}{\gamma}}$, we note that for all $\gamma<1$ (convex

\footnotetext{
${ }^{17} \mathrm{~A}$ number of papers further investigated the properties of skewness, kurtosis, and tail events in different settings (e.g. Chang et al. (2013) focus on market skewness and the cross-section of stock returns).

${ }^{18}$ In the empirical section we replicate Ranaldo and Favre (2003) results and further discuss issues related to the linear approach of Harvey and Siddique (2000) in the hedge fund performance setting.
} 
function), $1=E[m] \geq\left(1+\gamma E\left[\lambda_{\gamma}^{\prime} \tilde{\mathbf{R}}\right]\right)^{\frac{1}{\gamma}}$, which in turn implies that the hedge fund average $E\left[R_{i}^{H F}\right]$ is pinned down in the alpha computation. The opposite effect happens for $\gamma>1$. The linearity of the Hansen-Jagannathan SDF implies that the corresponding weight is exactly equal to one when $\gamma=1$.

Looking at the weights assigned to the higher-order mixed moments, we note that they are linked both to the Cressie-Read parameter $\gamma$ as well as to the optimal $\lambda_{\gamma}$. From the SDF estimation, we have that $1+\gamma \lambda_{\gamma}^{\prime} \tilde{\mathbf{R}}$ is nonnegative. This implies that, $1+\gamma E\left[\lambda_{\gamma}^{\prime} \tilde{\mathbf{R}}\right] \equiv 1-\gamma E[W]$ is also non-negative. Therefore, the signs of the weights are determined only by $\gamma$. In particular, $\gamma>1(\gamma<1)$ implies a negative (positive) weight for co-skewness. As usual, when $\gamma=1$ the estimator reduces to the Hansen and Jagannathan case, and all higher-order mixed moments are disregarded. Furthermore, the signs associated with weights on co-kurtosis are provided by a deterministic quadratic term on $\gamma$. Therefore, except for the region where $\gamma \in[0.5,1]$ the weights on co-kurtosis are all negative.

To give an idea of the behavior of these weights under the different settings we adopt in the paper, Figure 1 plots the estimated weights for five different sets of basis assets: (i) the CRSP value-weighted market portfolio, (ii) the Carhart (1997) four-factor model, (iii) the Fung and Hsieh (2001) underlying factors (the five factors that generate the trend-following strategies), (iv) the Fung and Hsieh (2001) ten factor model, and (v) the five underlying factors from Fung and Hsieh with the addition of the option portfolios fromAgarwal and Naik $(2004)^{19}$. The figure is based on the full sample for the underlying factors (January 1994 to June 2015) and a grid of $\gamma=\{-4,-3.5, \ldots, 4\}$ with 0.5 increments. It contains four panels, each indicating the weights for the hedge-fund average return, covariance, co-skewness, and co-kurtosis respectively.

Starting with the weights on the hedge-fund average return, Figure 1 exemplifies the effects of Jensen's inequality. Note that for all $\gamma$ s different from 1 we have a significant difference in weights depending on the benchmark factors. Figure 2 shows that the biggest differences in weights are explained by the differences in the expected excess return of the endogenous portfolio $\left(E\left[-\lambda_{\gamma}^{\prime} \tilde{\mathbf{R}}\right]\right)$ as a function of the different benchmark factors. In particular, the introduction of the trend-following or option factors generates a much higher variation of $E\left[-\lambda_{\gamma}^{\prime} \tilde{\mathbf{R}}\right]$ as a function of $\gamma$ than when only linear benchmark factors

\footnotetext{
${ }^{19}$ The specific factors in each model are defined in section 5.2.
} 
are adopted. Correspondingly in Figure 1, we note that when $\gamma<1$ the introduction of the trend-following or option factors significantly reduces the weights on the average hedge fund return. In aggregate, this reduction can be as high as 15\%. Additionally, the plot is steepest between $-2<\gamma<1$, revealing that the biggest effects of the nonlinearities occur in this region. The same analysis holds when $\gamma>1$. However, in this case, the Jensen's inequality works the other way around: the alphas are increased in comparison with the average hedge fund return.

The relationship between the hedge-fund alpha and the covariance with the basis assets is also interesting. Here we note that, across all values of $\gamma$, the weights assigned to this moment are monotonic. In particular, for all negative values this weight is higher than minus one and somewhat stable across different sets of basis assets. The picture changes radically for positive values: in this case, the introduction of the trend following or option factors significantly amplifies the weights.

Finally, the weights on co-skewness and co-kurtosis behave as expected: (i) both are zero for the Hansen and Jagannathan case, (ii) for co-skewness (co-kurtosis) they are a decreasing (parabolic) function, and (iii) introducing the trend-following or option factors significantly distorts these weights for all values of $\gamma$, with a higher effect for values greater than 1. More importantly, for $\gamma \leq 0$ the signs of the weights agree with theory: investors like skewness and dislike kurtosis.

\subsection{A Comparison with the Hansen-Jagannathan Estimator}

Chen and Knez (1996) propose an empirical application relying on the Hansen and Jagannathan (1991) stochastic discount factor to evaluate mutual fund performance. In this section, we illustrate empirically the differences between the Cressie-Read risk neutralization and that implied by Hansen and Jagannathan (1991) estimator. We tailor our examples to illustrate the effects of higher-order moments on both the stochastic discount factor estimation and the performance measurement.

We borrow from Almeida et al. (2017) and replicate their analysis using the full sample of Fung and Hsieh (2001) factors to estimate the Cressie-Read implied SDFs. Figure 3 plots the estimated risk neutral densities for three values of $\gamma,-3.5,-0.5,1$. The value $\gamma=1$ collapses to the Hansen and Jagannathan estimator and $\gamma=-0.5$ is the Hellinger estimator. We also select $\gamma=-3.5$ because this estimator gives substantial 
weight to skewness and kurtosis as seen in the Taylor expansion. Overall, the results in this picture are quite instructive: while the Hansen and Jagannathan estimator is linear in the returns both the Hellinger measure and $\gamma=-3.5$ generate non-trivial hyperbolic nonlinearities ${ }^{20}$. Additionally, the risk neutral density for the negative gammas puts significant more weight on adverse outcomes of the portfolio. This property translates to an important feature when measuring performance: for negative values of gamma, hedge funds performance can be significantly increased (penalized) if the fund generates returns that are positively (negatively) correlated with the SDF.

We now address the effect on the non-linearities implied by the different Cressie-Read estimators on the hedge fund alphas. For simplicity, we consider a model where the only benchmark factor is the market return $R_{M}$ (CAPM), simulated from a Student-t distribution. We simulate two hedge fund returns $R_{i}^{H F}, i=1,2$, from two different distributions (Normal and Student-t), but we maintain the same mean $E\left(R^{H F}\right)$, variance $\operatorname{var}\left(R^{H F}\right)$ and covariance $\operatorname{cov}\left(R^{H F}, R_{M}\right)$ with the market return for both funds. Therefore, by construction, each hedge fund is assigned the same OLS alpha $E\left(R^{H F}-R_{F}\right)-$ $\frac{\operatorname{cov}\left(R^{H F}, R_{M}\right)}{\operatorname{var}\left(R_{M}\right)} E\left(R_{M}-R_{F}\right)$, where $R_{F}=1$ is the risk-free rate. Therefore, it is quite clear that the linear model is not able to capture the higher-order moments of the hedge fund distributions. As long as the correlation structure between the two hedge fund returns and the factor in the model remains equal, the estimated alphas for the hedge funds are identical $^{21}$.

We apply the nonparametric methodology of the previous sections to the simulated data for a grid of $\gamma s$ from $[-5,3]$ with 0.5 increments. The point-wise estimated alphas and the bootstrapped confidence intervals (discussed further in section 4.4) are plotted in Figure 4. The solid line and the dashed line feature the point-wise alphas when hedge fund returns follow a normal and a Student-t distribution respectively. The alpha estimates for negative values of gamma differ substantially between the two hedge funds. For extreme negative values of $\gamma$ the higher-order moments from the Student-t distribution of the market return play a significant role. Indeed, one can see how the difference becomes larger as we move towards more negative values of $\gamma$ illustrating the difference in risk

\footnotetext{
${ }^{20}$ Note that when we refer to returns, we mean the endogenous portfolio returns, i.e., $-\lambda_{\gamma}^{\prime} \tilde{\mathbf{R}}$.

${ }^{21}$ Note that, even in more general linear factor models, if the correlation structure between the two hedge funds and the underlying factors is the same then they should have identical alphas (assuming here that the hedge funds have the same mean and standard deviation).
} 
compensation across SDFs. As expected, when $\gamma=1$, the Hansen and Jagannathan case, both hedge funds are assigned the same performance since the measure only takes into account the first and second moments of the benchmark factor $\left(R_{M}\right)$ and linear interactions between $R_{M}$ and $R_{i}^{H F}$ to calculate the alpha of each hedge fund. It is clear that the large differences across alphas given by different Cressie Read performance measures come from the higher-order properties of the Student-t distribution of the market return and from co-moments of this distribution with the hedge fund simulated returns as observed in section 3.1 .

The benchmark alpha for evaluating fund performance remains the Jensen's alpha obtained by an OLS regression of a fund's returns on the risk factors. The constrained Hansen-Jagannathan estimator will only differ from the OLS one for some particular sets of benchmark factors where in some states of nature, negative SDF values are set to zero. As it will be clear in the empirical section, even in these cases it turns out that the contribution of the $E\left[m^{\gamma=1} \epsilon_{i}\right]$ is small.

\section{Estimation and Tests of the Performance Measures}

Our measures are based on the computation of the Lagrange multipliers $\lambda_{\gamma}$ associated with the dual optimization problem in Corollary 1. In this section, we develop a consistent estimator for these parameters and derive its asymptotic distribution. We then infer the distribution of the implied performance measures $\alpha_{i}^{\gamma}$ in (15) based on the SDFs defined in Corollary 2 and computed with the estimated $\hat{\lambda}_{\gamma}$. Particular emphasis is given to deriving the asymptotic variance of $\alpha^{\gamma}$ to test whether the performance is significantly different from zero.

\subsection{Definitions}

In order to derive the estimator of the Lagrange multipliers $\hat{\lambda}_{T}^{22}$ and its asymptotic properties, we need to define functions that will enter in the objective function to be maximized (20), as well as their first and second derivatives. For each fixed $\gamma$ in the family of Cressie-Read discrepancies, let us define the following functions:

\footnotetext{
${ }^{22}$ For clarity of expressions in this section we drop in $\lambda_{\gamma}$ the subscript $\gamma$ denoting a specific member of the Cressie Read family and sometimes include the subscript $T$ denoting the sample size.
} 


$$
\begin{gathered}
f^{\gamma}(\lambda, \tilde{\mathbf{R}})=\frac{\left(1+\gamma \lambda^{\prime} \tilde{\mathbf{R}}\right)^{\frac{\gamma+1}{\gamma}}}{\gamma+1} \\
\mathfrak{M}^{\gamma}(\lambda, \tilde{\mathbf{R}})=E\left[f^{\gamma}(\lambda, \tilde{\mathbf{R}})\right]
\end{gathered}
$$

with its sample version, $\mathfrak{M}_{T}^{\gamma}(\lambda, R)$ :

$$
\mathfrak{M}_{T}^{\gamma}(\lambda, \tilde{\mathbf{R}})=\sum_{t=1}^{T} \frac{1}{T}\left[\frac{\left(1+\gamma \lambda^{\prime} \tilde{\mathbf{R}}_{t}\right)^{\frac{\gamma+1}{\gamma}}}{\gamma+1}\right]
$$

To prove the asymptotic normality of the estimator we will need the Hessian and the Fisher Information matrices that we denote by $H_{\lambda \lambda}(\lambda, \tilde{\mathbf{R}})$ and $S_{\lambda}(\lambda, \tilde{\mathbf{R}})$ respectively.

The first derivative of $f^{\gamma}(\lambda, R)$ is given by:

$$
\begin{aligned}
h^{\gamma}(\lambda, \tilde{\mathbf{R}}) & =\frac{\partial}{\partial \lambda^{\prime}} f^{\gamma}(\lambda, \tilde{\mathbf{R}}) \\
& =\left(1+\gamma \lambda^{\prime} \tilde{\mathbf{R}}\right)^{\frac{1}{\gamma}} \tilde{\mathbf{R}}
\end{aligned}
$$

from which we can calculate the score vector:

$$
\begin{aligned}
U_{T}(\lambda, \tilde{\mathbf{R}})=\frac{\partial \mathfrak{M}_{T}^{\gamma}(\lambda, \tilde{\mathbf{R}})}{\partial \lambda^{\prime}} & =\frac{1}{T} \sum_{t=1}^{T} h^{\gamma}\left(\lambda, \tilde{\mathbf{R}}_{t}\right) \\
& =\frac{1}{T} \sum_{t=1}^{T}\left(1+\gamma \lambda^{\prime} \tilde{\mathbf{R}}_{t}\right)^{\frac{1}{\gamma}} \tilde{\mathbf{R}}_{t}
\end{aligned}
$$

The expectation of the cross-product of the score vector by its transpose provides the Fisher information matrix $S_{\lambda}$ :

$$
S_{\lambda}=E\left(U_{T}(\lambda, \tilde{\mathbf{R}}) U_{T}(\lambda, \tilde{\mathbf{R}})^{\prime}\right)
$$

The Hessian matrix $H_{\lambda \lambda}$ is given by:

$$
\begin{aligned}
H_{\lambda \lambda} & =\frac{\partial^{2} \mathfrak{M} \gamma(\lambda, \tilde{\mathbf{R}})}{\partial \lambda^{\prime} \partial \lambda^{\prime}} \\
& =E\left(\left(1+\gamma \lambda^{\prime} \tilde{\mathbf{R}}\right)^{\left.\frac{1-\gamma}{\gamma} \tilde{\mathbf{R}} \tilde{\mathbf{R}}^{\prime}\right),}\right.
\end{aligned}
$$

and its corresponding sample version $H_{\lambda \lambda, T}$ by: 


$$
\begin{aligned}
H_{\lambda \lambda, T} & =\frac{\partial^{2} \mathfrak{M}_{T}^{\gamma}(\lambda, \tilde{\mathbf{R}})}{\partial \lambda^{\prime} \partial \lambda^{\prime}} \\
& =\frac{1}{T} \sum_{t=1}^{T}\left(1+\gamma \lambda^{\prime} \tilde{\mathbf{R}}_{t}\right)^{\frac{1-\gamma}{\gamma}} \tilde{\mathbf{R}}_{t} \tilde{\mathbf{R}}_{t}^{\prime}
\end{aligned}
$$

We are also interested in characterizing the estimators when the SDF achieves zeros in some states of nature, which may be the case for any member in the Cressie Read family with parameter $\gamma>0$. To that end, Let $f^{\gamma+}(\lambda, \tilde{\mathbf{R}})$ denote the following restricted version of $f^{\gamma}(\lambda, \tilde{\mathbf{R}})$ :

$$
f^{\gamma+}(\lambda, \tilde{\mathbf{R}})=\left[\frac{\left(1+\gamma \lambda^{\prime} \tilde{\mathbf{R}}\right)^{\frac{\gamma+1}{\gamma}}}{\gamma+1}\right] I_{\left\{1+\gamma \lambda^{\prime} \tilde{\mathbf{R}} \geq 0\right\}}
$$

We also define a restricted version of $\mathfrak{M}^{\gamma}(\lambda, \tilde{\mathbf{R}})$ :

$$
\mathfrak{M}^{\gamma+}(\lambda, \tilde{\mathbf{R}})=E\left[f^{\gamma+}(\lambda, \tilde{\mathbf{R}})\right]
$$

and its corresponding sample version given by:

$$
\mathfrak{M}_{T}^{\gamma+}(\lambda, \tilde{\mathbf{R}})=\sum_{t=1}^{T} \frac{1}{T}\left\{\left[\frac{\left(1+\gamma \lambda^{\prime} \tilde{\mathbf{R}}_{t}\right)^{\frac{\gamma+1}{\gamma}}}{\gamma+1}\right] I_{\left\{1+\gamma \lambda^{\prime} \tilde{\mathbf{R}}_{t} \geq 0\right\}}\right\}
$$

Finally, let $H_{\lambda \lambda}^{+}(\lambda, \tilde{\mathbf{R}})$ and $S_{\lambda}^{+}(\lambda, \tilde{\mathbf{R}})$ denote restricted versions of the Hessian and Fisher Information matrices, and $H_{\lambda \lambda, T}^{+}$the corresponding sample version of $H_{\lambda \lambda}^{+}(\lambda, \tilde{\mathbf{R}})$.

\subsection{Estimator of the Lagrange Multiplier Vector and its Asymp- totic Properties}

Almeida and Garcia (2012) use Cressie-Read discrepancies to estimate parametric models under the assumption of misspecification. Their procedure comprised the joint estimation of the parameters of the models and of the Lagrange multipliers associated with the dual problem. They proved the consistency of the estimator for the whole parameter vector and derived its asymptotic distribution. Our problem is simpler since we have to estimate only the Lagrange multiplier vector. Therefore we simplify their approach to address the more specific estimation problem.

First we need to state two assumptions in order to establish the convergence in probability of our estimator (Theorem 1) and derive its asymptotic distribution (Theorem 
2).

Assumption 1. We assume that $\lambda^{*}$ is the unique solution to the following problem:

$$
\lambda^{*}=\underset{\lambda \in \mathbb{R}^{K}}{\operatorname{argmin}} \mathfrak{M}^{\gamma}(\lambda, \tilde{\mathbf{R}})
$$

Assumption 1 guarantees that the problem is well specified. This hypothesis parallels Assumption (1.A) in Newey and Smith (2004). Under the assumption that $E\left[\tilde{\mathbf{R}} \tilde{\mathbf{R}}^{T}\right]$ is non-singular, the Hessian of $-\mathfrak{M}^{\gamma}$ is non-singular and negative definite. Thus, $-\mathfrak{M}^{\gamma}$ is strictly concave in $\lambda$. This, coupled with the fact that $\lambda \in \mathbb{R}^{K}$, is sufficient to guarantee the uniqueness of $\lambda^{* 23}$.

Using the notation defined in the previous section, the sample version of the dual parameters $\hat{\lambda}_{T}$ can be estimated as follows:

$$
\hat{\lambda}_{T}=\underset{\lambda \in \mathbb{R}^{K}}{\operatorname{argmin}} \mathfrak{M}_{T}^{\gamma}(\lambda, \tilde{\mathbf{R}})
$$

\section{Assumption 2.}

(A) The process $\tilde{\mathbf{R}}_{t}$ is stationary and ergodic.

(B) The process $\tilde{\mathbf{R}}_{t}$ is strongly mixing with mixing coefficients $\alpha_{t}$ satisfying $\sum_{t=1}^{\infty} \alpha_{n}^{1-1 / b}<$ $\infty$ and $b>1$.

(C) $E\left[\tilde{\mathbf{R}} \tilde{\mathbf{R}}^{T}\right]$ is non-singular.

(D) $\operatorname{Var}\left(\sqrt{T}\left[\frac{\partial}{\partial \lambda} \mathfrak{M}_{T}^{\gamma}\left(\lambda^{*}, \tilde{\mathbf{R}}\right)\right]\right) \rightarrow_{p} S_{\lambda}>0$ when $T \rightarrow \infty$.

(E) $\mathfrak{M}^{\gamma}(\lambda, \tilde{\mathbf{R}})<\infty$ for all vectors $\lambda$ in a neighborhood of $\lambda^{*}$.

(F) $H_{\lambda \lambda}$ is of full rank, and for any $\lambda \in \mathbb{R}^{K}$, almost surely continuous as a function of $\tilde{\mathbf{R}}$.

Assumptions (A) and (B) guarantee that the basis assets returns are well-behaved. In particular assumption (B) ensures that, given "sufficient time" between groups of sequential observations of $\tilde{\mathbf{R}}_{t}$, these groups are independent. Assumption (C) guarantees the absence of multicollinearity. Assumptions (D) to (F) are based on Kitamura and

\footnotetext{
${ }^{23}$ We focus on the case $E[m]=1$, which is the one considered in our empirical applications. All the results generalize to the case where $E[m]=a, a \in \mathbb{R}$.
} 
Stutzer (1997) and allow us to apply a weak version of the law of large numbers and the Central Limit theorem (CLT). In particular, (D) guarantees that the variance in the CLT is well-defined (covariance stationarity condition), (E) can be relaxed with additional algebraic costs (see comments in Kitamura and Stutzer (1997)). (F) allows us to prove the asymptotic convergence of $H_{\lambda \lambda, T}^{-1}$ to $H_{\lambda \lambda}^{-1}$ by relying on the Continuous Mapping Theorem.

Theorem 1. Under Assumption 2, $\hat{\lambda}_{T}$ converges in probability to $\lambda^{*}$.

The proof of this theorem is available in Almeida and Garcia (2012) and relies on an application of Theorem 2.7 of Newey and McFadden (1994).

Theorem 2. Under Assumption 2 we have that:

$$
\sqrt{T}\left(\hat{\lambda}_{T}-\lambda^{*}\right) \rightarrow_{d} N\left(0, V_{\lambda}\right)
$$

where $V_{\lambda}$ is given by $H_{\lambda \lambda}^{-1} S_{\lambda} H_{\lambda \lambda}^{-1}$.

See proof in the Appendix.

\subsection{Imposing The Non-Negativity Constraint on the SDF}

From Almeida and Garcia (2017) we know that imposing non-negativity restrictions has different implications depending on three regions for the parameter $\gamma$ that indexes the Cressie-Read family: (i) $\gamma>0$. (ii) $\gamma<0$ and (iii) $\gamma=0$ (see Corollary 1).

Starting with $\gamma=0$, we have an unconstrained maximization problem. This comes directly from the fact that when $\gamma=0$ the Cressie-Read family converges to the exponential tilting case, which is positive by construction. Thus, in this particular case, the consistency of the estimator as well as the asymptotic normality follow directly from the results in the previous section.

The second case is when $\gamma<0$. In this region for $\gamma$, the optimization function for $\lambda$ is modified to be able to restrict the SDF to be strictly positive. Corollary 1 reveals that this restriction introduces a $\delta$ set-indicator function that takes value zero if $\left(1+\gamma \lambda^{\prime} \tilde{\mathbf{R}}\right)>0$ and $\infty$ otherwise. To prove consistency and asymptotic normality we must guarantee the stability of the objective function near the solution. Here it is sufficient that $\mathfrak{M}^{\gamma}(\lambda, \tilde{\mathbf{R}})<\infty$ for all vectors $\lambda$ in a neighborhood of $\lambda^{*}$. Note that this is exactly what 
is stated in Assumption $2(\mathrm{E})^{24}$. Since there is an open neighborhood $\tilde{\Gamma}\left(\lambda^{*}, \tilde{\epsilon}\right)$ around $\lambda^{*}$ where $\delta(x \mid A)=0$, asymptotically the central arguments of the previous section hold ${ }^{25}$.

Finally, when $\gamma>0$ we lose the point-wise differentiability of the objective function. The objective function is affected by an indicator function for the set $\left(1+\gamma \lambda^{\prime} \tilde{\mathbf{R}}\right) \geq 0$, which introduces a "kink" when the equality holds. Below we modify the previous framework to allow us to still prove the asymptotic properties. For the consistency result the main problem is to establish the validity of condition (iv) in Newey and McFadden (1994)'s theorem. The basic idea for the normality convergence is to find an approximation different from the Taylor expansion that holds even when we lose differentiability. To that end, we extend results provided by Li et al. (2010) on the non-negatively constrained Hansen and Jagannathan estimator to deal with the whole positive side of the CressieRead family indexed by $\gamma>0$. In the Appendix, we provide the corresponding modified assumptions, denoted by Assumption 3, needed to prove consistency and asymptotic normality of the Lagrange multipliers on the $\gamma>0$ case.

Theorem 3. Under Assumption $3, \hat{\lambda}_{T}$ converges in probability to $\lambda^{*}$.

See proof in the Appendix.

For asymptotic normality, we need two lemmas stated below and proved in the Appendix. In what follows, let $E_{T}(X)=\frac{1}{T} \sum_{t=1}^{T} X_{t}$ represent the empirical sample average of a random variable.

Lemma 1. If Assumption 3 holds, we can write the following relation:

$$
\begin{aligned}
E_{T}\left[f^{\gamma+}(\lambda, \tilde{\mathbf{R}})\right] & =E\left[f^{\gamma+}\left(\lambda^{*}, \tilde{\mathbf{R}}\right)\right]+\left(E_{T}-E\right)\left[f^{\gamma+}\left(\lambda^{*}, \tilde{\mathbf{R}}\right)\right]+\tilde{S}_{\lambda}^{+^{\prime}}\left(\lambda-\lambda^{*}\right) \\
& +\frac{1}{2}\left(\lambda-\lambda^{*}\right)^{\prime} H_{\lambda \lambda}^{+}\left(\lambda-\lambda^{*}\right)+o\left(\left\|\lambda-\lambda^{*}\right\|\right)+o_{p}\left(\left\|\lambda-\lambda^{*}\right\| T^{-1 / 2}\right),
\end{aligned}
$$

where $\tilde{S}_{\lambda}^{+}=\left(E_{T}-E\right)\left[\frac{\partial f^{\gamma+}(\lambda, \tilde{\mathbf{R}})}{\partial \lambda}\right]$ and $H_{\lambda \lambda}^{+}=E\left[\frac{\partial^{2} f^{\gamma+}(\lambda, \tilde{\mathbf{R}})}{\partial \lambda \partial \lambda^{\prime}}\right]$.

Lemma 2. If Assumption 3 holds, the following asymptotic approximation is valid:

\footnotetext{
${ }^{24}$ When $\mathfrak{M}^{\gamma}(\cdot, \tilde{\mathbf{R}})$ is a continuous function of the Lagrange Multipliers, which is the case with the Cressie Read family, this condition is automatically satisfied.

${ }^{25}$ From an implementation viewpoint, within the sphere $\tilde{\Gamma}\left(\lambda^{*}, \tilde{\epsilon}\right)$ we can evaluate the objective function by dropping the delta term.
} 


$$
\begin{aligned}
\max _{\lambda \in \mathbb{R}^{K}} E_{T}\left[f^{\gamma+}(\lambda, \tilde{\mathbf{R}})\right] & =E\left[f^{\gamma+}(\lambda, \tilde{\mathbf{R}})\right]+\left(E_{T}-E\right)\left[f^{\gamma+}(\lambda, \tilde{\mathbf{R}})\right] \\
& -\frac{1}{2} \tilde{S}_{\lambda}^{+^{\prime}} H_{\lambda \lambda}^{+} \tilde{S}_{\lambda}^{+}+o_{p}\left(T^{-1}\right)
\end{aligned}
$$

where $\tilde{S}_{\lambda}^{+}$and $H_{\lambda \lambda}^{+}$were defined in Lemma 1 .

Now, using these two lemmas, we can establish asymptotic normality of the estimator $\hat{\lambda}_{T}$.

Theorem 4. If Assumption 3 holds, $\hat{\lambda}_{T}$ has the following asymptotic distribution:

$$
\sqrt{T}\left(\hat{\lambda}_{T}-\lambda^{*}\right) \rightarrow_{d} N\left(0, V_{\lambda}\right)
$$

where $V_{\lambda}$ is given by $\left(H_{\lambda \lambda}^{+}\right)^{-1} S_{\lambda}^{+}\left(H_{\lambda \lambda}^{+}\right)^{-1}$.

See proof in the Appendix.

\subsection{Alpha Performance Estimation and Testing}

A fundamental question in fund performance evaluation is to determine whether the performance measure $\alpha$ of a given fund is significantly different from zero. In the linear APT/Jensen framework this is accomplished with a simple Student-t test. In our context, given an estimated SDF $m^{\gamma}$ and a hedge fund return time series $\left\{R_{i t}^{H F}\right\}_{t=1, \ldots T}$, we estimate its performance by simply calculating $\left.\alpha_{i}^{\gamma} \equiv \alpha^{\gamma}\left(R_{i}^{H F}, \hat{\lambda}_{\gamma}\right)=\frac{1}{T} \sum_{t=1}^{T}\left[m_{t}^{\gamma} R_{i t}^{H F}-1\right)\right]$. The question becomes: how to test whether $\alpha_{i}^{\gamma}=0$ ? One simple way to proceed is to define the following statistic (Chen and Knez, 1996):

$$
\mathcal{H}_{T}=T w_{T}\left(\alpha_{i}^{\gamma}\right)^{2}
$$

where $w_{T}$ is the inverse of the variance of $\alpha_{i}^{\gamma}$. Additionally, note that we explicitly write $\alpha_{i}^{\gamma}$ as dependent on the first-step estimate $\hat{\lambda}_{\gamma}$ of $\lambda^{*}$. Therefore $w_{T}$ should account for the uncertainty attached to the estimates of the vector of parameters $\lambda^{*}$. Rewriting the sample estimate of $\alpha_{i}^{\gamma}$ taking into account the SDF estimation we have that:

(i) If $\gamma>0$ :

$$
\alpha_{i}^{\gamma}=\frac{1}{T} \sum_{t=1}^{T}\left[\left(1+\gamma \hat{\lambda}_{\gamma}^{\prime}\left(\mathbf{R}_{t}-1_{K}\right)\right)^{\frac{1}{\gamma}} I_{\Lambda\left(\mathbf{R}_{t}\right)}\left(\hat{\lambda}_{\gamma}\right) R_{i, t}^{H F}-1\right]
$$


(ii) If $\gamma<0$ :

$$
\alpha_{i}^{\gamma}=\frac{1}{T} \sum_{t=1}^{T}\left[\left(1+\gamma \hat{\lambda}_{\gamma}^{\prime}\left(\mathbf{R}_{t}-1_{K}\right)\right)^{\frac{1}{\gamma}} R_{i, t}^{H F}-1\right]
$$

(iii) If $\gamma=0$ :

$$
\alpha_{i}^{\gamma}=\frac{1}{T} \sum_{t=1}^{T}\left[\exp \left(\hat{\lambda}_{0}^{\prime}\left(\mathbf{R}_{t}-1_{K}\right)\right) R_{i, t}^{H F}-1\right]
$$

Using the above representation of the Cressie-Read performance measures, we observe the explicit dependence of $\alpha_{i}^{\gamma}$ on $\left\{\mathbf{R}, R_{i}^{H F}, \hat{\lambda}_{\gamma}\right\}$. Hence, by applying the delta method we can compute the variance $w_{T}$ as follows:

$$
\left(w_{T}\right)^{-1} \equiv \operatorname{Var}\left(\alpha_{i}^{\gamma}\right)=\frac{\partial \alpha_{i}^{\gamma^{\prime}}}{\partial \lambda} \operatorname{Var}(\lambda) \frac{\partial \alpha_{i}^{\gamma}}{\partial \lambda}
$$

where:

$$
\frac{\partial \alpha_{i}^{\gamma}}{\partial \lambda}=\frac{1}{T} \sum_{t=1}^{T} \frac{\partial m_{t}^{\gamma}}{\partial \lambda} R_{i t}^{H F}
$$

and,

(i) $\frac{\partial m_{t}^{\gamma}}{\partial \lambda}=\left(\mathbf{R}_{t}-1_{K}\right)\left(1+\gamma \hat{\lambda}_{\gamma}^{\prime}\left(\mathbf{R}_{t}-1_{K}\right)\right)^{\frac{1-\gamma}{\gamma}} I_{\Lambda\left(\mathbf{R}_{t}\right)}\left(\hat{\lambda}_{\gamma}\right)$, if $\gamma>0$,

(ii) $\frac{\partial m_{t}^{\gamma}}{\partial \lambda}=\left(\mathbf{R}_{t}-1_{K}\right)\left(1+\gamma \hat{\lambda}_{\gamma}^{\prime}\left(\mathbf{R}_{t}-1_{K}\right)\right)^{\frac{1-\gamma}{\gamma}}$, if $\gamma<0$,

(iii) $\frac{\partial m_{t}^{\gamma}}{\partial \lambda}=\left(\mathbf{R}_{t}-1_{K}\right) \exp \left(\hat{\lambda}_{0}^{\prime}\left(\mathbf{R}_{t}-1_{K}\right)\right)$, If $\gamma=0$.

Under the null of zero alpha, $\mathcal{H}_{T}$ converges asymptotically to a $\chi^{2}$ distribution with one degree of freedom.

Despite our asymptotic results, when using hedge fund data, it is essential to account for the limited number of return observations typically available for a particular hedge fund. Therefore, we also rely on bootstrap to address the small sample issue. We apply a nonparametric bootstrap resampling technique (pairs bootstrap) where both the returns of the benchmark assets and the individual hedge fund are resampled together to compute the bootstrapped standard errors, following the methodology described in MacKinnon (2006). Given that most hedge fund time series do not share the same sample period 
we perform the bootstrap on a fund-by-fund basis. While this is costly in terms of computational time, our sample characteristics significantly restrict other methods (joint resampling of the full panel for instance). In the online appendix, we provide a detailed comparison between the asymptotic and the bootstrapped t-statistics and provide some results on the power of the asymptotic tests.

Instead of deriving the limiting distribution of the multipliers and of the alphas pointwise in $\gamma$, it would be interesting to see for what region of $\gamma$ the null of $\alpha(\gamma)=0$ is rejected and for what is not ${ }^{26}$. In the online appendix we detail how to derive the limiting distribution of the $\alpha(\gamma)$ as an empirical process over $\gamma \in \Gamma_{S} \subset \Gamma$.

\section{$5 \quad$ Hedge Fund Data and Filters}

It is well-known in the finance literature that commercial hedge fund databases suffer from severe biases (see Fung and Hsieh (2001), Aggarwal and Jorion (2010), Patton et al. (2015), Bollen and Pool (2009) and Aiken et al. (2013) among others). Over the years, researchers have applied stricter filters as it became obvious that some reporting issues were severely biasing the performance results. Even though we use the Lipper-TASS data set from Thomson Reuters, the problems we identified are in line with the former literature.

Traditionally, the literature focused on two primary sources of bias: survivorship and backfill (e.g. Fung and Hsieh (2001) and Aggarwal and Jorion (2010) respectively). Given the lack of regulation, fund managers not only can choose when to report their returns but also to which commercial database to report it. Fund managers also have the discretion to change previously reported returns (Patton et al., 2015). All these issues are carefully investigated in previous papers. Several conclusions can be drawn. The consensus consists of using data post-1994 when most commercial databases started to include defunct funds. This potentially alleviates the survivorship bias. To deal with the backfill bias two possibilities are proposed: to exclude the first twelve months (or 24 months) of returns for all funds (e.g. Bali et al. (2011)) or, as proposed by Aggarwal and Jorion (2010), to detect back-fillers according to the difference between the hedge fund inception date and the data it started to report to TASS (or any data provider).

\footnotetext{
${ }^{26}$ We thank an associate editor for this suggestion.
} 
In addition to the usual biases addressed in the literature, there is a growing effort to improve data quality in empirical applications. This is reflected in the number of papers investigating data biases and data problems in commercial databases (e.g. Patton et al. (2015) and Bollen and Pool (2009)). Despite this effort, recent articles raised additional concerns. Straumann (2009) found several problematic idiosyncrasies in many databases (including the one used in this paper). Specifically, the author found an alarming number of hedge funds with excessive zero returns, consecutive equal returns, and repeated "blocks" of returns. Building on this, Bollen and Pool (2012) investigated these data problems further and showed that several of them are indicative of future sanctions from the Security Exchange Commission (SEC).

In our online appendix, we carefully describe the filters we apply to the original data and explore the implications of the corrected data biases for performance measurement. Following and expanding Straumann (2009), we consider seven filters (in addition to the traditional ones): (1) funds with an abnormal number of zero returns; (2) funds with repeated blocks of returns (e.g. repetitions of the block A,B,C with $A, B, C \in \mathbb{R}$ ); (3) funds with an unusual amount of repeated returns (e.g. A, A, A); (4) funds whose returns calculated from reported net asset values (NAVs) do not coincide with reported net returns; (5) funds with rounded returns; (6) funds that do not report assets under management; (7) funds that share blocks of returns in the time series (e.g. two funds share the same returns for, say, Jan - 2000 to Dec - 2000).

\subsection{Summary Statistics for the Final Sample of Hedge Funds}

Table 1 provides descriptive statistics for the whole set of selected hedge funds for each year from 1994 to 2015 . We report the number of hedge funds in the sample at the beginning of the year, the number of entrants, the number of funds dissolved as well as the number of hedge funds at year-end and their total assets under management (in billions of dollars). Using all active hedge funds within a given year, we construct an equally-weighted portfolio and compute its mean, median, standard deviation as well as minimum and maximum returns ${ }^{27}$. Notably the number of hedge funds peaked around 2007 and fell sharply after the financial crisis. The same pattern applies to the assets

\footnotetext{
${ }^{27}$ Given the high number of hedge funds that do not declare their assets under management on a consistent basis an AUM-weighted portfolio is impractical.
} 
under management. In fact, by the end of 2014, the AUM figure was less than half its peak level observed in 2007. Overall, the mean and median returns are positive throughout the sample. The exceptions are, as expected, 2008 and 2011, two crisis years where a large number of hedge funds were dissolved.

Table 2 complements this analysis with information regarding the cross-section of hedge funds. Here we present the average return across funds, the average assets under management, fund age, management fee and performance fee. In contrast with the equally-weighted index, we note that the mean is lower for the individual hedge funds (0.57 versus a historical mean of 0.67 for the equally-weighted index). The same goes for the individual standard deviation. Nonetheless, the minimum (maximum) returns are significantly lower (higher) than those reported for the historical data appearing in Table 1. The fund age reveals an important piece of information for our empirical application: the median fund has only 71 months of observable returns. Therefore, dealing with small sample sizes is a first-order problem for inference. The median management and performance fees are close to the industry $2 / 20$ standard. Finally, we note that the median hedge fund manages about 30 million dollars. When compared with the mean value of 130 million dollars this fact reveals that a small number of funds manage most of the money. Overall our descriptive statistics are very close to Bali et al. (2011) who use a similar data set.

We construct eleven indexes based on the primary strategy reported by each hedge fund: Convertible Arbitrage, CTA, Emerging Markets, Equity Market Neutral, Event Driven, Fixed Income Arbitrage, Funds of Funds, Global Macro, Long-Short Equity Hedge, Managed Futures and Multi-Strategy. The summary statistics for these indexes are presented in Table 3. Overall, we note a significant difference in the average return across categories. While equity neutral hedge funds have an average return of $0.60 \%$ per month, long-short funds produce a monthly average return of about $0.93 \%$. The difference in standard deviation is even higher, ranging from 0.88 for the market-neutral index to 4.21 for the emerging market one. Consistent with previous papers we also document a negative skewness for most of the primary-strategy groups (similarly to Kosowski et al. (2007)). Regarding kurtosis, all the groups we analyze have distributions that are leptokurtic.

A significant portion of our paper focuses on the higher-order properties of hedge fund 
returns and their implications for performance measurement. Table 4 reports summary statistics (mean, standard deviation, skewness and kurtosis) for the cross-section of hedge funds segregated by their self-declared primary strategy. Overall, we note a significant difference in terms of average return across categories. While equity neutral hedge funds have an average return of $0.44 \%$ per month, long-short funds produce an average of about $0.76 \%$. Standard deviations are in general higher than for the indexes, and the difference in standard deviation across strategies ranges from 2.61 to 6.12 . In addition, similarly to the indexes case, we also document negative skewness and leptokurtic distributions for most of the primary-strategy groups. The magnitudes of the higher-order moments change substantially with respect to the corresponding statistics of the indexes in Table 3. Thus, our empirical analysis focuses both on individual and index-level performance measurement.

\subsection{Summary Statistics for the Factors}

In this paper, we stress two sources of nonlinearities and their implications for performance measurement: the individual or index hedge fund returns and the benchmark factor returns. Previous studies on hedge fund returns have typically used both linear and nonlinear factors. To this end, we include the linear factors of Carhart (1997) and two sets of factors explicitly designed to capture non-linear hedge fund strategies. First, we adopt the workhorse model of Fung and Hsieh (2001). The updated model consists of ten factors (see Fung's website). Five of these factors are based on primitive trend following strategies for bonds (PTFSBD), currencies (PTFSFX), commodities (PTFSCOM), interest rate (PTFSIR) and stocks (PTFSSTK). The complementary factors are the underlying portfolios used to construct the trend-following ones: the monthly returns on the S\&P 500, the spread between the Russell 2000 index total monthly returns and the S\&P 500 total monthly returns, the monthly total returns on the MSCI Emerging Markets index, the change in constant maturity yield 10-year Treasury bonds, and the change in the spread between Moody's Baa and the 10-year Treasury ${ }^{28} 29$.

\footnotetext{
${ }^{28}$ The underlying data are acquired trough Datastream using the "RI" code, from the Federal Board of Governors H15 forms and from St. Louis FRED dataset.

${ }^{29} \mathrm{An}$ anonymous referee suggested to replace the changes in Treasury yields and corporate spreads by total returns on a Treasuries index and High Yield corporate index. We re-estimated the Fung an Hsieh SDF with these new factors for the Hedge Fund indexes and reported the results in Tables A20 and A21 in the online appendix.
} 
Agarwal and Naik (2004) propose a new set of nonlinear factors constructed using option prices. We follow the authors and construct four portfolios of at and out of the money put and calls using raw data from Option Metrics. In our empirical applications with the option portfolios, we also add the same linear factors from Fung and Hsieh $(2001)^{30}$.

In Table 5 we report the summary statistics for all of the above-mentioned factors. Overall, the sample properties are fairly close to the original factors of Carhart (1997), Fung and Hsieh (2001), and Agarwal and Naik (2004). The statistics about primary equity and size risk factors are without surprise - a mean close to $10 \%$ for the S\&P 500 index and a spread of about $4 \%$ with the Russell index. The bond and the credit factors exhibit much lower means (between $-2 \%$ and $4 \%$ ). Most indexes exhibit little skewness and excess kurtosis. This is also the case for the Carhart (1997) factors. The picture is radically different for the trend-following factors constructed by Fung and Hsieh (2001). Means are sizable and typically negative. Additionally, the volatilities are substantially higher than those of the underlying factors. The picture for the option factors is even stronger: all means are extremely negative with substantial standard deviations. Common to both option and trend-following factors is the high skewness of the returns. In contrast, on average, the kurtosis is lower for these factors than for the underlying. The only exception is the interest rate trend-following strategy, which has an extremely high kurtosis.

\section{Empirical Findings}

In this section we apply the nonparametric measures defined in the previous sections to evaluate the performance of hedge fund indexes and individual hedge funds over the 1994 to 2015 period. We first apply the estimation and testing methodologies developed in Section 4 to identify the factors that are significant for evaluating performance depending on investors' preferences (proxied by our gamma parameter in the Cressie-Read family of measures). We then proceed to assess alpha performance based on the estimated SDFs. We test the hypothesis that the alphas are different from zero and measure their

\footnotetext{
${ }^{30}$ The SAS code to generate these factors is available upon request. Note that a version of the code is available through WRDS. This version does not take into account recent changes in the expiration schedule (see CBOE website).
} 
heterogeneity across sets of benchmark factors and strategies reported by the hedge funds. We pay particular attention to the performance contribution of the nonlinear exposures of hedge funds to risks. In particular we compare the effect of adding linearly higherorder moments of factor returns, as it is usually done, to our nonparametric approach that accounts functionally for the exposure to higher moments.

\subsection{SDF Estimation Results}

We compute the stochastic discount factor for different sets of basis assets (factors) and a range of values for $\gamma \mathrm{s}$. We use linear models that are workhorse models in the empirical finance literature (CAPM and Carhart four-factor models) and the two benchmark models in the hedge fund literature, Fung and Hsieh (2001) and Agarwal and Naik (2004), that include several asset classes and options as factors. For the Cressie-Read gammas, we consider the following $\gamma$ values: $\{-3.5,-2,-1,-0.5,0,0.5,1\}$. This set includes the HJ linear SDF with non-negativity constraint $(\gamma=1)$, a set of SDFs that give mild weights to skewness and kurtosis $(\gamma=-1,-0.5,0,0.5)$, and two SDFs that give larger absolute weights to skewness and kurtosis $(\gamma=-2$ and -3.5$)$. This range of $\gamma$ values is quite representative of the Cressie-Read family and generates substantial variability in both the estimated SDF as well as the hedge fund alphas. In particular, for $\gamma<<-3.5$, the implied SDF concentrates most of the probability density on a few states of nature. On the contrary, for values of $\gamma>>1$ the implied SDF assigns zero probabilities for several states of nature in order to correctly price the basis assets. Both these features are uninteresting for performance measurement, and thus we are comforted in the representativeness of investors' weighting of risks by the chosen $\gamma$ values.

For the full-sample estimation results, from January 1994 to June 2015, we discuss the estimated $\lambda \mathrm{s}$ and the time-series dynamics of the estimated SDFs. The sign of the lambdas indicates the position of the portfolio in the corresponding factors. Given the presence of a negative sign in the optimization problem in Corollary 1 a negative sign for lambda indicates a long position and vice versa. For space considerations, we include the estimation results in the main text only for the Agarwal and Naik model (in Table 6), which includes four options portfolios on the S\&P 500 along with the five indices described earlier. The portfolio with the highest $\lambda$ is precisely the $\mathrm{S} \& \mathrm{P} 500$ (the one with

the highest mean), but all other underlying assets are insignificant or only marginally 
significant across all $\gamma_{\mathrm{s}}$. For the options portfolios, the positions in the put options are particularly striking. Across all $\gamma_{\mathrm{s}}$ the investor shorts out-of-the-money puts and hedges out the position by buying at-the-money ones. This is consistent with the typical results that selling insurance is profitable on average. In addition, the $\lambda$ s associated with more negative $\gamma$ s are smaller in magnitude, which indicates that more risk averse agents reduce their exposure to this strategy given the possibility of rare disastrous outcomes. We should mention that the asymptotic and bootstrap t-statistics are close to each other and concur on the significance of the factors.

The results for the three other sets of factors are reported in the online Appendix. The results for the CAPM and Carhart models are reported in Tables A6 and A7. For both specifications, the market portfolio emerges as a statistically significant factor with a positive load. Second, the addition of the long-short portfolios, in the Carhart model, slightly increases the absolute value assigned to the market portfolio across all $\gamma \mathrm{s}$. In addition, the momentum portfolio also appears as a long and statistically significant factor. The estimated $\lambda_{\mathrm{s}}$ for the Fung and Hsieh model are reported in Table A8. First, two of the five underlying portfolios, i.e. the S\&P 500 and the credit spread, are held long and are statistically significant ${ }^{31}$. For the trend following strategies, only the S\&P 500 lookback straddle appears statistically significant.

Our methodology to estimate the SDF is based on pricing exactly the risk factors. We include in the aforementioned four tables the pricing error associated with each benchmark factor. The pricing errors are zero for most gamma values and benchmark factors. For $\gamma=0.5$, we observe very small pricing errors of the order of 0.05 basis points for five factors (three trend following, bond and emerging markets index in the Fung Hsieh dataset), and 0.1 basis points for the OTM CALL factor in the Agarwal and Naik (2004) dataset. For both $\gamma=0$ and $\gamma=-3.5$ we observe pricing errors of less than 0.1 basis point for only one benchmark each (respectively market and size spread) out of 19 benchmark factors. Therefore a zero alpha performance for a fund will rightfully be indicative of a static exposure to the factors.

In Figure 5, we plot the time series of the estimated SDFs for three selected values of $\gamma(1,0$ and -3.5$)$ and for the four sets of basis assets. It appears clearly that the SDFs without trend-following and options factors are much less volatile across all $\gamma$ s.

\footnotetext{
${ }^{31}$ Market is statistically significant for both asymptotic and bootstrap t-statistics while credit spread only for the asymptotic t-statistics.
} 
Within the same set of basis assets, SDFs obtained with more negative gammas exhibit a higher volatility. The SDFs with trend following and options portfolios also feature large peaks at times of financial market stress (1998, 2002, 2006 and during the recent financial crisis). The events are also visible in the SDFs without these portfolios but with much less amplitude. Note finally the zero values assigned to some states of nature in the Hansen and Jagannathan SDF (most notably in the figure for the Fung and Hsieh set of factors).

\subsection{Analyzing Hedge Fund Indexes}

Hedge fund indexes are constructed according to the primary strategy reported by individual hedge funds. Although pooling hedge fund returns hide some significant heterogeneity among the funds, we gain in two dimensions. First, the hedge fund indexes allow us to construct portfolios with observed returns for the whole available sample. This is in clear contrast with the median 71 observations for the individual hedge funds. Second, by pooling the data, we are better able to contrast the performance across combinations of $\gamma$ and benchmark assets since aggregate results are easier to present and interpret.

\subsubsection{Alpha Performance}

For each hedge fund index, we calculate the full sample alpha as well as the associated asymptotic and bootstrapped t-statistics. For completeness, we compare our estimates to the Jensen's alpha, the standard to evaluate fund performance ${ }^{32}$.

We focus mainly on the Fung and Hsieh set of factors because it contains the most diverse exposures both in terms of asset indices and option-like trend-following factors. We report the results in Table 7). Except for the convertible arbitrage index, all estimates for the Jensen's alpha are positive and statistically significant ${ }^{33}$, but the Cressie-Read alphas offer a different picture. For example, for the CTA and the Managed Futures indexes, we note a regular increase in the point-wise alpha as $\gamma$ becomes more negative. The funds in this category invest in financial and commodity futures markets and currency

\footnotetext{
${ }^{32}$ For the Jensen's alpha, the bootstrapped t-statistics are calculated using the residual bootstrap proposed by Kosowski et al. (2006).

${ }^{33}$ The OLS $R^{2} \mathrm{~s}$, not reported in the table, increase substantially with the introduction of the Fung and Hsieh non-linear factors in comparison to the CAPM model (54\% versus 30\%).
} 
markets around the world. A large proportion are trend followers (buy in an up market and sell in a down market). This indicates that these funds provide investors with positive returns in bad times, when their marginal utility is high. The same pattern is present for convertible arbitrage but the magnitude of the alpha difference is not as marked.

The emerging markets category provides an excellent example of how non-linearities introduced by the Cressie-Read estimates might alter the investors' evaluation of performance. The Jensen's alpha obtained with the Fung and Hsieh factors is positive, statistically significant. However, the Cressie-Read estimates decrease as $\gamma$ becomes more negative and are not significantly different from zero. For most other categories the alphas are relative uniform across all values of $\gamma$ and their statistical significance is also similar according to both asymptotic and bootstrap t-statistics.

We report the alpha point estimates and their t-statistics for the CAPM, Carhart and Agarwal and Naik models in Tables A9, A10 and A11 of the online appendix, respectively. The first two sets of factors are missing the options exposures and the last one misses options factors related to fixed income, commodities, and currencies. Because of this, the results show a higher heterogeneity in performance and statistical significance across the various values of $\gamma^{34}$. For several strategies, we observe patterns that are very different from those generated by the Fung and Hsieh factors. CTA and Managed Futures are increasing with $\gamma$, since the options factors are only related to the S\&P 500. The alpha for the Funds of Funds category is reduced by half for the three sets of factors with respect to the Fung and Hsieh factors and is not statistically different from zero according to the bootstrap statistics. This is also the case for Global Macro for the Agarwal and Naik set of factors. The variation between sets of factors is also interesting for the Long-short equity hedge. Since the long-short strategies can be based on value, growth, or size, the Carhart set is the only one that shows that this strategy can provide positive returns in bad times since the alpha is higher for more negative values of $\gamma$.

To sum up, it is clear that the choice of benchmark factors matters crucially for evaluating hedge fund performance. The heterogeneity in performance evaluation is enhanced by the Cressie-Read measures with respect to the two linear measures. However, imposing non-negativity in the Hansen and Jagannathan measure does not change performance much compared to the Jensen's alpha. These findings illustrate that the nonlinear ex-

\footnotetext{
${ }^{34}$ Note that the non-negatively constrained Hansen and Jagannathan and the OLS alphas are extremely close to each other.
} 
posures in the hedge funds strategies are evaluated differently by investors with different attitudes towards asymmetry or tail risks.

In our analysis of the performance of hedge fund indexes, we have reported the results for each index and for selected values of $\gamma$. We compare the magnitude and the sign of the difference in alpha between the linear Jensen's alpha and the nonlinear Cressie-Read alpha. We observe that for some strategies, the performances concur for all values of $\gamma$, while for others the negative values of $\gamma$ change the sign or increase or decrease the alpha substantially with respect to the Jensen's alpha. If we were interested in testing whether the alpha performance of a gamma-measure was superior to the Jensen's alpha considered as a benchmark, then the reality check proposed by White (2000) would allow us to evaluate the distribution of the alpha performance measure by giving consideration to the full set of strategies that led to the best-performing measure ${ }^{35}$.

In the next section, we add a comparison between an extended linear approach by which higher moments of a portfolio returns are added to the alpha regression to capture co-skewness and co-kurtosis to our nonlinear SDF approach.

\subsubsection{Implications of Higher-Order Moments and SDF Non-Linearities}

To analyze how the non-linearities affect the alpha estimates, we choose a simple CAPM factor model ${ }^{36}$. We proceed by adding polynomial terms to the market factor in the linear regression, as follows ${ }^{37}$ :

$$
\tilde{R}_{i, t}^{H F}=\alpha_{i}+\beta_{i, R_{M}}\left(R_{M, t}-R_{F, t}\right)+\sum_{p=1}^{P} \beta_{i, p}\left(R_{M, t}-R_{F, t}\right)^{p+1}+\epsilon_{i, t} .
$$

$\tilde{R}_{i, t}^{H F}$ denotes the excess return for fund $i$ and $R_{M, t}-R_{F, t}$ the return of the market

\footnotetext{
${ }^{35}$ The test will be based on the difference between $D^{\gamma}=\alpha^{\gamma}-\alpha_{J}$, where $\alpha_{J}$ stands for the Jensen's alpha. For each hedge fund strategy, we will test the hypothesis $H_{0}: V=\max _{\gamma \in \Gamma_{s}} D^{\gamma} \leq 0$, where $\Gamma_{s}=$ $[-3.5,-2,-1,-0.5,0,0.5,1]$. We will compute the statistic $V$ with the data and $V_{i}, i=1, \ldots, B$, over B boostrap samples. The bootstrap method will be the nonparametric bootstrap resampling technique (pairs bootstrap) where both the returns of the benchmark assets and the individual hedge fund are resampled together to compute the statistic $V_{i}$. We compare $V$ to the quantiles of $V_{i}$ to obtain White's reality check p-value for the null hypothesis.

${ }^{36}$ In the hedge fund performance measurement literature, the typical approach relies on multiple factors, often the Fung and Hsieh 10 factors. Introducing polynomial terms with these more elaborate models is unfeasible. In contrast, as seen in our Taylor expansion, our methodology provides a parsimonious higher-order exposure to an optimally chosen linear combination of all factors in the model. Therefore, the number of factors is not a problem in our approach.

${ }^{37}$ This is similar to Agarwal et al. (2008), who investigate if higher moments of equity risk explain hedge fund returns.
} 
portfolio in excess of the risk-free rate. The addition of polynomial terms is similar to the models proposed by Harvey and Siddique (2000) for co-skewness and Ang et al. (2006) for co-kurtosis ${ }^{38}$. We want to contrast the performance evaluation based on this linear addition of non-linear terms to the performance implied by our SDF approach where the non-linearities are weighted differently by diverse investors.

Table 8 puts forward the similarities and differences between the two approaches. It is informative to compare the fourth-order linear model, which incorporates all higher-order moments up to the fourth power, to the -3.5 value for $\gamma$ in our nonparametric approach. The two corresponding alphas are similar, positive and significantly different from zero for strategies that are immune from market risk, Equity market neutral, Long-short equity hedge, Event driven and Multi-strategy. In fact for these categories all specifications give about the same performance assessment. It means that the strategies are market risk neutral to many moments (see Patton (2009)). The two measures also agree in attributing zero performance to the Convertible arbitrage and Funds of funds categories, in contrast to all the other measures that do not penalize the high kurtosis associated with these strategies.

The differences between the two measures are also striking. For CTA, Global Macro and Managed Futures, the fourth-order specification attributes a zero-performance while the -3.5 gamma estimates a positive and significant alpha. The latter measure likes the positive skewness and the low kurtosis of these strategies. In contrast, introducing the power terms in the linear regression reduces to zero the positive performance implied by the CAPM. Finally for the Emerging markets, the Cressie-Read measure inverts the positive alpha estimated with the fourth-order specification and puts it to a value of zero both numerically and statistically.

A significant difference between the two approaches is that, for all indexes, we note a monotonic relationship between the alphas and gammas in the SDF approach. It is not the case for the polynomial approach. Moreover, the introduction of the polynomial terms in the linear framework suppresses the typical "excess return" interpretation of the OLS alpha. Our stochastic discount factor methodology naturally embeds different degrees of nonlinearities and preserves the interpretation of the alphas as the risk-adjusted hedge fund return. Furthermore, cross-correlation terms are already taken into account in the

\footnotetext{
${ }^{38}$ Ranaldo and Favre (2003) incorporate these higher-order moments to evaluate the performance of hedge funds and show that they are relevant for some funds.
} 
$\lambda$ 's estimation, allowing us to expand significantly the number of benchmarks used to capture the nonlinearities in assessing performance.

\subsection{Analyzing Individual Hedge Funds}

To obtain the alpha of each hedge fund, we need to use a computationally-intensive method. We need to match the sample period where the fund was alive to estimate the SDF from the benchmark factors. If we were to estimate the SDF for the full sample, we would use information that might not have been available during the period the fund existed. Also, considering a sub-sample of an admissible SDF estimated with the full sample will not be an admissible SDF anymore because it will not price exactly the factors.

\subsubsection{Individual Hedge Fund Performance}

To capture the richness of results for individual alphas, we provide summary statistics, draw kernel densities of alphas and their t-statistics, test for the difference of performance between the linear and nonlinear estimators, compare fund rankings across estimators and analyze investors' divergence about fund performance.

6.3.1.1 Summary statistics Table 9 presents, for all four sets of basis assets and all gamma values, the mean, standard deviation, skewness, kurtosis and the percentage of alphas that are positive and statistically significant at the $10 \%$ level, based on bootstrapped t-statistics. Similarly to hedge fund indexes, the average alpha estimate for the CAPM model is quite similar across $\gamma \mathrm{s}$. However, across all performance measures, the alpha estimates are larger for the two sets with nonlinear factors. Note also the difference in the percentage of statistically significant alphas, from $22 \%$ for the linear/OLS measure to $11 \%$ for $\gamma=-3.5$ in the Fung and Hsieh set of factors. Finally, the Agarwal and Naik model increases substantially the variance of the cross-sectional alphas. The inclusion of option strategies in the factor model generates alpha distributions with positive skewness and higher kurtosis. Nonetheless, the percentages of significant alphas are similar to that of the Fung and Hsieh model. 


\subsubsection{Comparisons of Cross-sectional Alpha Distributions between Estima-}

tors In Table 10, we report a number of quantiles of the cross-sectional distributions of all fund alphas for two sets of factors (Carhart and Agarwal and Naik) and the set of positive and negative gamma values. The patterns for the two sets of factors are similar but the distribution for the set including options returns is more widespread. Quantitatively the alphas for the $1 \%, 90 \%$ and $99 \%$ show significant differences between the two sets. The mean spread, averaged across the values of gamma, is about 35 basis points per month at the $1^{\text {th }}$ percentile, 20 basis points per month at the $90^{\text {th }}$ percentile and 100 basis points per month for the $99^{\text {th }}$ percentile. Ignoring the use of options severely underestimates the alphas of the top funds and overestimates those of the bottom funds.

To further illustrate the differences between the distributions, Figure 6 presents the estimated kernel density of the difference between the Cressie-Read and Jensen's alpha for each fund, all $\gamma$ values and the Carhart, Fung and Hsieh and Agarwal and Naik sets of factors ${ }^{39}$. Overall, results behave as expected: (i) when we include the nonlinear factors the heterogeneity increases substantially, in comparison with the Carhart model; (ii) moving away from the Hansen and Jagannathan case further increases this heterogeneity. In particular, for negative $\gamma s$, it is not rare to find funds with a difference between OLS/linear and Cressie Read alphas of $0.25 \%$ per month, a sizable difference when considering that the median Jensen's alpha is about $0.20 \%$ per month. Note that this difference allows us to capture the disagreement about fund performance between investors as well as measure the influence of the higher-order moments on the difference between the Cressie-Read alpha and the OLS one.

To formally test whether this difference in performance is statistically significant we implement a paired mean test. Figure 7 plots the cross-sectional kernel density of the estimated t-statistics for each set of benchmark factors across four panels. As expected, for the CAPM and Carhart models, most estimated alpha differences are insignificant since higher-order moments play a minor role. However, when we include the nonlinear factors in the Fung and Hsieh and Agarwal and Naik models, the variance of the crosssectional distribution of the t-statistics increases substantially. In fact, for the later model, when $\gamma=-3.5$, almost $10 \%$ of the funds have Cressie-Read alphas whose difference to

\footnotetext{
${ }^{39}$ For the CAPM and the Hansen and Jagannathan estimators, the heterogeneity is quite small, making it harder to visualize the densities.
} 
their OLS counterparts are positive and statistically significant ${ }^{40}$. As before, for values of $\gamma$ close to 1 the alpha difference becomes less significant.

To analyze the cross-sectional fund performance across strategies, we choose the Fung and Hsieh basis assets. In Table 11, we report for all categories of funds the average and the $10 \%$ and $90 \%$ quantile alphas. A first unsurprising finding is that the average alpha across all categories and estimators is less than the corresponding index ones ${ }^{41}$. For the individual funds we average the alphas computed over different sample periods while for the index we compute the discounted aggregate return over the full sample. The variation of the average alpha over the estimators often parallels the variation observed for the indexes but not always (convertible arbitrage and CTA are good examples). The inter-quantile range tends to be larger for the negative gammas that weight more bad states of nature penalizing bad funds.

\subsubsection{T-statistics Comparisons between Estimators Figure 8 plots the cross-} sectional kernel densities of the asymptotic t-statistics for the four sets of factors, three values of gamma and the Jensen's alpha. The Cressie-Read cross-sectional t-statistics are much more conservative than the OLS ones, but are very similar to one another. The only significant difference is for the Agarwal and Naik estimates when $\gamma=-3.5$. In this case, the cross-sectional distribution is slightly more positively skewed than for the other gamma values.

Figure 9 plots the same set of figures for the bootstrapped t-statistics ${ }^{42}$. Interestingly, the bootstrap distribution of the OLS alpha t-statistics is much closer to the CressieRead alphas. This reveals that the asymptotic approximation in the OLS case bias the t-statistics estimate upward. Focusing on the bottom two panels of Figure 9 we note that the Hansen and Jagannathan bootstrapped t-statistics distribution is much more concentrated around zero. Again, this arises from the fact that when $\gamma>0$ the SDF may assume a zero value in some states of nature and biases the alpha estimates towards zero,

\footnotetext{
${ }^{40}$ Note that having a difference in performance (between any Cressie Read and Jensen measures) that is statistically significant does not imply that the absolute performance provided by each measure is statistically significant.

${ }^{41}$ One exception is for the negative gammas in the event driven category.

${ }^{42}$ Note that to bootstrap the OLS alpha we adopt the procedure of Kosowski et al. (2006), while for our procedure we rely on a non-parametric bootstrap. One advantage of our method, in comparison with Kosowski et al. (2006), is that our approach re-samples both the hedge fund returns as well as the underlying factors. Thus, we partially accommodate the critiques in Fama and French (2010) regarding the joint sample of fund's returns and factors.
} 
implying a more concentrated distribution.

6.3.1.4 Fund Ranking Comparisons between Estimators The comparisons of alpha quantiles or distributions for the cross-sections of funds point to the absolute performance differences between the various estimators but do not tell us how a particular fund fares relatively to others (its performance rank) and how this rank changes from one value of gamma to another. Table 12 gives a first answer to this question by showing the regular correlations and rank correlations of alpha measures for each pair of gamma values. We consider the two sets of factors that include non-linear factors (Fung and Hsieh and Agarwal and Naik). Correlations are reported in the lower triangular matrices while rank correlations are featured in the upper triangular matrices. Overall, the main message is similar for both sets of factors. Correlations and rank correlations are positive and decreasing as we move away from the more negative values to the positive values of gamma and to the Jensen's measure. The magnitudes are somewhat lower for the Agarwal and Naik factors but are aligned with the values of the Fung and Hsieh factors $^{43}$.

To further illustrate the differences in ranking between the measures at the individual fund level, Figure 10 plots densities of the ranking differences between the Jensen's alphas and the average of the negative Cressie-Read measures for the four sets of factors. Starting with the CAPM, we see that the density is relatively concentrated around zero but the tails appear fatter than for a normal distribution. The tails get thicker with the other sets of factors, first with the Carhart but mainly for the two sets with nonlinear factors. The probabilities are almost uniformly distributed for these two sets, with ranking differences of 300 almost as frequent as a zero difference. This important divergence shows that using the Jensen's alpha to measure performance will not suit all investors, especially when the fund returns have strong nonlinear exposures to the main sources of risk.

6.3.1.5 Investors' Divergence about fund performance To capture the substantial variation in the fund-by-fund alpha estimates across estimators, we propose a divergence measure Div $_{i}$ defined as the logarithm of the alpha variance for each fund

\footnotetext{
${ }^{43}$ The main difference is a slight increase in correlations for the Jensen's alpha and negative Cressie Read performance measures in the Fung and Hsieh set of factors when compared to the Agarwal and Naik set.
} 
across $\gamma \mathrm{s}$. The median alpha variance for the two sets of nonlinear factors is in the order of $33 \%$. To better understand the variables that drive this divergence between investors, we run the following regression:

$$
\operatorname{Div}_{i}=\beta_{0}+\sum_{i=1}^{N} \beta_{i} X_{i}+\epsilon_{i}
$$

where $X_{i}$ includes both individual fund characteristics and co-moments of the funds with the market, which we have identified as the most significant risk factor. As fund characteristics, we include the mean return, the skewness and kurtosis of the fund returns, the fund idiosyncratic volatility ${ }^{44}$ and the age and size of the fund. We add the co-skewness and co-kurtosis of each hedge fund with the market return (CRSP value-weighted portfolio), one lag of the fund returns to control for smoothing as well as strategy dummies.

We report the regression results in Table 13 for the four sets of basis assets. Except for the CAPM, we note that the higher the hedge fund mean, the lower the disagreement between investors. For the CAPM and Carhart sets of factors, both co-skewness and cokurtosis increase strongly and significantly the divergence. Theoretically, this is explained by the different loads on these variables depending on the gammas used in the estimation (see Figure 1). However the introduction of nonlinear option-return factors in the Fung and Hsieh and Agarwal and Naik sets captures the nonlinear exposure of the funds with respect to the market and risk neutralizes the fund returns with respect to higher moments of market returns. Therefore the alphas for the various measures and the differences between them is less sensitive to these higher co-moments ${ }^{45}$. For similar reasons the skewness and kurtosis of the fund returns do not explain the divergence. Apart from the fund mean, the main explanatory factors are the idiosyncratic volatility, which increases the disagreement, and the age of the fund, which decreases divergence since it signals quality.

\subsubsection{How should an investor choose his performance measure? In our} approach the curvature of the dual portfolio objective function will change according to

\footnotetext{
${ }^{44}$ We measure the idiosyncratic volatility according to the Carhart factors as it is done in the literature. Our results also hold for both the Fung and Hsieh factors as well as the Agarwal and Naik factors.

${ }^{45}$ This is definitely the case for the Agarwal and Naik set of factors, since all the options are on the equity market. For the Fung and Hsieh factors the coefficient of co-skewness is bigger and statistically significant but the lookback options are on many primary sources of risk not solely the equity market.
} 
each gamma. Therefore the performance evaluation will be different for each gamma and the ranking of funds will change according to each gamma. This begs the question of how does an investor identify the gamma that corresponds to the risk adjustment measure he will be comfortable with. We suggest below a procedure that could be followed by a potential hedge fund investor.

1. The problem of an investor is to add one or several hedge funds to manage the risk of his/her current portfolio. He has to determine with which gamma he should evaluate the performance of the candidate hedge funds and the share(s) of the selected hedge fund(s) he should include in his new portfolio.

2. The first step is to determine the benchmark assets to use to evaluate performance. This should correspond to the asset classes included in his or her current portfolio.

3. To choose the $\gamma$ to use, the investor can compute the weights of the optimal portfolio for each $\gamma$ with the selected set of factors. He will choose the $\gamma$ that implies weights closest to his current portfolio weights according to a chosen metric (for instance Euclidean) given these benchmark assets.

4. The investor will then select a number of candidate funds and evaluate their performance with the SDF implied by the selected $\gamma$ and the benchmark assets.

5. The investor can then rank the funds, select a few top performers and see how well they will perform on a sample left out of the estimation process.

6. The last step is to determine how much of one or several funds to add to the current portfolio. We suggest to include the returns of the selected funds in the previous step with the benchmark asset returns and solve the dual problem to obtain the optimal portfolio weights.

Given the length and the focus of the paper, we leave the implementation of such a procedure for future research.

\section{Conclusion}

In this article, we build on and expand the performance measure methodology proposed by Chen and Knez (1996). Under this general framework, we propose a novel 
class of performance measures based on Cressie-Read discrepancies that are easy to estimate, satisfy no-arbitrage restrictions, and contains both theoretical and empirical desirable properties. These performance measures encompass the known non-negatively constrained Hansen and Jagannathan estimator explored in Chen and Knez (1996) and Li et al. (2010). Additionally, we demonstrate that departing from the quadratic case (HJ) allows our performance measure to embed information about the higher-order mixed moments of the hedge fund return with respect to the benchmark factors adopted in the stochastic discount factor estimation.

Concerning hypothesis testing, we extend Almeida and Garcia (2012) and provide a full set of asymptotic results for both the Lagrange multipliers, associated with the stochastic discount factor estimation, as well as the performance measure. Given the known non-normality of both hedge fund returns as well as the traditional hedge fund benchmarks (e.g. Fung and Hsieh (2001)) we propose a nonparametric bootstrap to test for the statistical significance of the individual hedge fund alpha estimates.

Empirically, we illustrate our methodology using a large set of individual hedge fund data. We pay particular attention to the filtering of the raw data by combining a large set of filters. We show that neglecting these filters might severely bias upwards the evaluation of performance. Our results reveal a sizable heterogeneity in the funds' valuation depending on the loadings on higher-order moments. In particular, we often find hedge funds with statistically significant OLS alphas but insignificant, and even negative alphas, for our nonparametric estimators. Moreover, we find meaningful economic differences between performance measures both in absolute terms as well as in relative terms (the cross-section ranking of the hedge funds).

\section{Acknowledgments}

We would like to thank an anonymous referee, Yacine Aït-Sahalia, Walter Distaso (discussant), Ravi Jagannathan, Robert Korajczyk, Hugues Langlois (discussant), Guillaume Roussellet (discussant) and seminar participants at Princeton University, the Hedge Fund Conference of the Oxford-Man Institute of Quantitative Finance (2011), EDHEC-Risk Days Europe in London (2012), EDHEC-Risk Days Asia in Singapore (2012), 5th Annual SOFIE conference in Oxford (2012), SMU-ESSEC Symposium on Empirical Finance 
and Financial Econometrics in Singapore (2012), Inquire UK Seminar in Bath, Toulouse Financial Econometrics Conference (2012), 13th Brazilian Meeting of Finance (2013), 11th Annual SOFIE conference in Lugano (2018), 10th Annual Hedge Fund and Private Equity Research Conference in Paris (2018), CIREQ Econometrics Conference in Montreal (2018) as well as seminar participants at Princeton, CASS Business School, EDHEC Business School, CREST (Paris), GREQAM (Marseille) and Kellogg School of Management. The first author acknowledges financial support from CNPq. The second author acknowledges financial support from ANBIMA and FAPERJ. He is also thankful to Viktor Todorov, Torben Andersen and the Kellogg School of Management for their hospitality during part of the development of this research project. The third author is a research Fellow of CIRANO and CIREQ. He acknowledges financial support from the SSHRC Insight Funding program. 


\section{References}

Agarwal, V., G. Bakshi, and J. Huij (2008). Do higher-moment equity risks explain hedge fund returns? Working papers, J. Mack Robinson College of Business, Georgia State University.

Agarwal, V. and N. Y. Naik (2004). Risks and portfolio decisions involving hedge funds. Review of Financial Studies 17(1), 63-98.

Aggarwal, R. and P. Jorion (2010). The performance of emerging hedge funds and managers. Journal of Financial Economics 96(2), 238-256.

Aiken, A. L., C. P. Clifford, and J. Ellis (2013). Out of the dark: Hedge fund reporting biases and commercial databases. Review of Financial Studies 26(1), 208-243.

Almeida, C., K. Ardison, R. Garcia, and J. Vicente (2017). Nonparametric tail risk, stock returns, and the macroeconomy. Journal of Financial Econometrics 15(3), 333-376.

Almeida, C. and R. Garcia (2012). Assessing misspecified asset pricing models with empirical likelihood estimators. Journal of Econometrics 170, 519-537.

Almeida, C. and R. Garcia (2017). Economic implications of nonlinear pricing kernels. Management Science 63(10), 3361-3380.

Ang, A., J. Chen, and Y. Xing (2006). Downside risk. Review of Financial Studies 19(4), 1191-1239.

Backus, D., M. Chernov, and I. Martin (2011, December). Disasters implied by equity index options. The Journal of Finance 66(6), 1969-2011.

Backus, D., M. Chernov, and S. Zin (2014). Sources of entropy in representative agent models. Journal of Finance 69, 51-99.

Bali, T. G., S. Brown, and M. O. Caglayan (2011). Do hedge funds' exposures to risk factors predict their future returns? Journal of Financial Economics 101(1), 36-68.

Bansal, R. and B. Lehmann (1997). Growth-optimal portfolio restrictions on asset pricing models. Macroeconomic Dynamics (1), 333-54. 
Beja, A. (1971). The structure of the cost of capital under uncertainty. Review of Economic Studies 38(3), 359-368.

Billio, M., G. Jannin, B. Maillet, and L. Pelizzon (2013). Portfolio performance measure and a new generalized utility-based n-moment measure. Working Papers 2013:22, Department of Economics, University of Venice "Ca' Foscari".

Bollen, N. P. and V. K. Pool (2009). Do hedge fund managers misreport returns? evidence from the pooled distribution. Journal of Finance 64(5), 2257-2288.

Bollen, N. P. B. and V. K. Pool (2012). Suspicious patterns in hedge fund returns and the risk of fraud. Review of Financial Studies 25(9), 2673-2702.

Capocci, D. and G. Hubner (2004). Analysis of hedge fund performance. Journal of Empirical Finance 11(1), 55 - 89.

Carhart, M. (1997). On persistence in mutual fund performance. Journal of Finance 52(1), 57-82.

Chang, B. Y., P. Christoffersen, and K. Jacobs (2013). Market skewness risk and the cross section of stock returns. Journal of Financial Economics 107(1), 46-68.

Chen, Z. and P. J. Knez (1996). Portfolio performance measurement: Theory and applications. Review of Financial Studies 9(2), 511-55.

Cochrane, J. (2001). Asset pricing. Princeton [u.a.]: Princeton Univ. Press.

Corcoran, S. A. (1998). Bartlett adjustment of empirical discrepancy statistics. Biometrika 85, 967-972.

de Roon, F. and P. Karehnke (2017). Spanning tests for assets with option-like payoffs: the case of hedge funds. Technical report, Tillburg University.

Dittmar, R. (2002). Nonlinear pricing kernels, kurtosis preference, and evidence from the cross section of equity returns. Journal of Finance 57(1), 369-403.

Fama, E. and K. French (1993). Common risk factors in the returns on stocks and bonds. Journal of Financial Economics 33(1), 3-56. 
Fama, E. F. and K. R. French (2010). Luck versus skill in the cross-section of mutual fund returns. Journal of Finance 65(5), 1915-1947.

Farnsworth, H., W. E. Ferson, D. Jackson, and S. Todd (2002, February). Performance Evaluation with Stochastic Discount Factors. NBER Working Papers 8791, National Bureau of Economic Research, Inc.

Ferson, W. and H. Mo (2016). Performance measurement with selectivity, market and volatility timing. Journal of Financial Economics 121(1), 93-110.

Ferson, W. E. (2013). Investment performance: A review and synthesis. Volume 2, Chapter 14, pp. 969-1010. Elsevier.

Fung, W. and D. A. Hsieh (2001). The risk in hedge fund strategies: Theory and evidence from trend followers. Review of Financial Studies 14(2), 313-41.

Goetzmann, W., J. Ingersoll, M. Spiegel, and I. Welch (2007). Portfolio performance manipulation and manipulation-proof performance measures. Review of Financial Studies $20(5), 1503-46$.

Hansen, L. and R. Jagannathan (1991). Implications of security market data for models of dynamic economies. Journal of Political Economy 99(2), 225-62.

Harvey, C. and A. Siddique (2000). Conditional skewness in asset pricing tests. Journal of Finance 55(3), 1263-1295.

Hubner, G., M. Lambert, and N. Papageorgiou (2015). Higher-moment risk exposures in hedge funds. European Financial Management 21(2), 236-264.

Jurek, J. and E. Stafford (2015). The cost of capital for alternative investments. Journal of Finance 5(70), 2185-2226.

Kimball, M. (1993). Standard risk aversion. Econometrica 61(3), 589-611.

Kitamura, Y. and M. Stutzer (1997). An information-theoretic alternative to generalized method of moments estimation. Econometrica 65(4), 861-874.

Kosowski, R. (2011). Do mutual funds perform when it matters most to investors? us mutual fund performance and risk in recessions and expansions. Quarterly Journal of Finance 1(3), 607-664. 
Kosowski, R., N. Y. Naik, and M. Teo (2007). Do hedge funds deliver alpha? a bayesian and bootstrap analysis. Journal of Financial Economics 84(1), 229-264.

Kosowski, R., A. Timmermann, R. Wermers, and H. White (2006). Can mutual fund "stars" really pick stocks? new evidence from a bootstrap analysis. Journal of Finance 61(6), 2551-2595.

Kraus, A. and R. H. Litzenberger (1976). Skewness preference and the valuation of risk assets. Journal of Finance 31(4), 1085-1100.

Li, H., Y. Xu, and X. Zhang (2010, August). Evaluating asset pricing models using the second Hansen-Jagannathan distance. Journal of Financial Economics 97(2), 279-301.

Li, H., Y. Xu, and X. Zhang (2016). Hedge fund performance evaluation under the stochastic discount factor framework. Journal of Financial and Quantitative Analysis $51(01), 231-257$.

MacKinnon, J. (2006). Bootstrap methods in econometrics. Economic Record 82, s2-s18.

Mas-Colell, A., M. D. Whinston, and J. R. Green (1995). Microeconomic Theory. New York: Oxford University Press.

Newey, W. and R. Smith (2004). Higher order properties of gmm and generalized empirical likelihood estimators. Econometrica 72(1), 219-255.

Newey, W. and K. West (1987). A simple, positive semi-definite, heteroskedasticity and autocorrelation consistent covariance matrix. Econometrica 55(3), 703-08.

Newey, W. K. and D. McFadden (1994, January). Large sample estimation and hypothesis testing. In R. F. Engle and D. McFadden (Eds.), Handbook of Econometrics, Volume 4 of Handbook of Econometrics, Chapter 36, pp. 2111-2245. Elsevier.

Patton, A. J. (2009). Are "market neutral" hedge funds really market neutral? Review of Financial Studies 22, 2495-2530.

Patton, A. J., T. Ramadorai, and M. Streatfield (2015). Change you can believe in? hedge fund data revisions. Journal of Finance 70(3), 963-999. 
Ranaldo, A. and L. Favre (2003). How to price hedge funds: From two- to four-moment capm. Technical report, EDHEC.

Straumann, D. (2009). Measuring the quality of hedge fund data. The Journal of Alternative Investments 12(2), 26 - 40.

Stutzer, M. (1996). A simple nonparametric approach to derivative security valuation. The Journal of Finance 51(5), 1633-1652.

White, H. (2000). A reality check for data snooping. Econometrica 68(5), 1097-1126. 


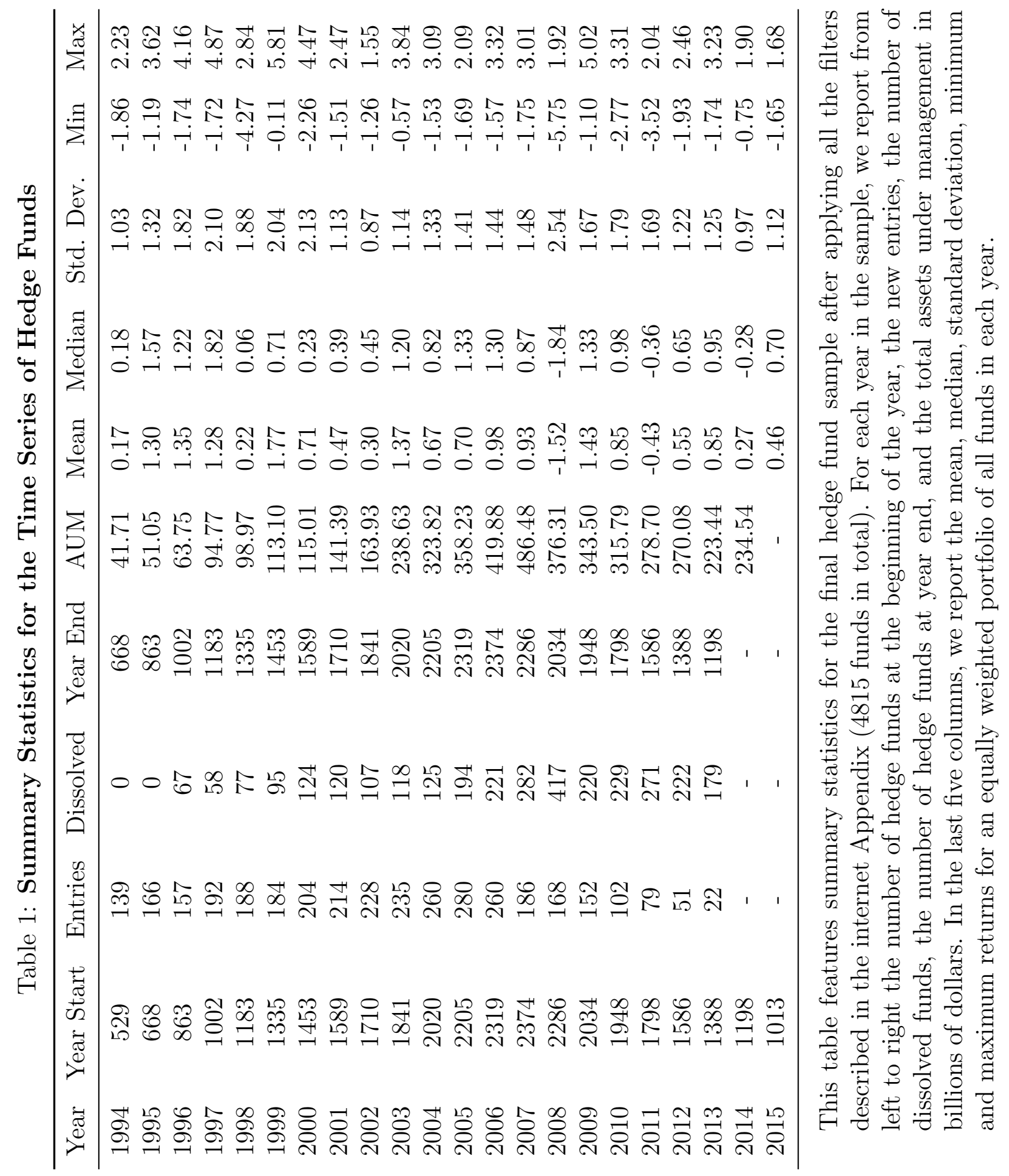


Table 2: Summary Statistics for the Cross-section of Hedge Funds

\begin{tabular}{lcccccc}
\hline & $\mathrm{N}$ & Mean & Median & Std. Dev. & Min & Max \\
\hline Avg. Ret & 4815 & 0.57 & 0.53 & 0.83 & -8.76 & 18.30 \\
Avg. AUM & 4815 & 0.13 & 0.03 & 0.41 & 0.00 & 17.44 \\
Age & 4815 & 85.43 & 71.00 & 53.52 & 24.00 & 258.00 \\
Man. Fee & 4806 & 1.50 & 1.50 & 0.72 & 0.00 & 8.00 \\
Perf. Fee & 4789 & 16.02 & 20.00 & 7.47 & 0.00 & 50.00 \\
\hline
\end{tabular}

This table features cross-sectional summary statistics for the final hedge fund sample after applying all the filters described in the internet Appendix (4815 funds in total). From left to right, we report the mean, median, standard deviation, minimum and maximum of the average hedge fund returns (we compute the average return for each hedge fund during the year and then we compute the statistics, as well as the averages for the assets under management, age, management fees and performance fees).

Table 3: Summary Statistics for Strategy Indexes

\begin{tabular}{lccccc}
\hline Category & $\mathrm{N}$ & Mean & SD & Skew & Kurtosis \\
\hline Convertible Arbitrage & 103 & 0.61 & 2.20 & -2.40 & 22.54 \\
CTA & 412 & 0.62 & 2.07 & 0.45 & 3.25 \\
Emerging Markets & 354 & 0.81 & 4.21 & -1.08 & 7.93 \\
Equity Market Neutral & 176 & 0.60 & 0.88 & -0.37 & 5.66 \\
Event Driven & 310 & 0.77 & 1.74 & -1.51 & 8.61 \\
Fixed Income Arbitrage & 113 & 0.61 & 1.38 & -2.87 & 19.27 \\
Fund of Funds & 1222 & 0.47 & 1.61 & -0.48 & 5.61 \\
Global Macro & 187 & 0.65 & 1.72 & 0.76 & 4.48 \\
Long/Short Equity Hedge & 1162 & 0.93 & 2.71 & -0.26 & 4.41 \\
Managed Futures & 351 & 0.64 & 2.89 & 0.28 & 2.80 \\
Multi-Strategy & 244 & 0.72 & 1.38 & -0.82 & 5.43 \\
\hline
\end{tabular}

This table features summary statistics (number of funds, mean, standard deviation, skewness and kurtosis) for the equallyweighted indexes of each strategy based on the final hedge fund sample after applying all the filters described in the internet Appendix. 
Table 4: Summary Statistics for the Cross-section of Hedge Funds Returns by Strategy

\begin{tabular}{lccccc}
\hline Category & $\mathrm{N}$ & Mean & Std. Dev. & Skewness & Kurtosis \\
\hline Convertible Arbitrage & 103 & 0.53 & 2.61 & -0.85 & 10.59 \\
CTA & 412 & 0.65 & 5.70 & 0.37 & 5.03 \\
Emerging Markets & 354 & 0.60 & 6.12 & -0.41 & 7.79 \\
Equity Market Neutral & 176 & 0.44 & 2.43 & -0.22 & 6.47 \\
Event Driven & 310 & 0.73 & 3.00 & -0.46 & 7.44 \\
Fixed Income Arbitrage & 113 & 0.53 & 2.47 & -1.40 & 14.45 \\
Funds of Funds & 1222 & 0.36 & 2.67 & -0.75 & 7.25 \\
Global Macro & 187 & 0.58 & 4.26 & 0.24 & 6.29 \\
Long/Short Equity Hedge & 1162 & 0.76 & 4.98 & -0.03 & 5.56 \\
Managed Futures & 351 & 0.53 & 5.44 & 0.13 & 5.61 \\
Multi-Strategy & 244 & 0.52 & 3.24 & -0.42 & 8.18 \\
\hline
\end{tabular}

This table features summary statistics (number, mean, standard deviation, skewness and kurtosis) for each category of hedge funds based on their self-reported strategy for the final hedge fund sample after applying all the filters described in the internet Appendix. All statistics are calculated at the fund level and then averaged across funds. 
Table 5: Summary Statistics for the Benchmark Risk Factors

\begin{tabular}{lccccccc}
\hline & Mean & Median & Std. Dev. & Min & Max & Skewness & Kurtosis \\
\hline MKT & 0.63 & 1.33 & 4.42 & -17.23 & 11.35 & -0.74 & 4.19 \\
SMB & 0.17 & 0.01 & 3.40 & -17.17 & 22.08 & 0.81 & 11.76 \\
HML & 0.20 & -0.01 & 3.11 & -11.25 & 12.91 & 0.09 & 5.80 \\
MOM & 0.45 & 0.58 & 5.16 & -34.58 & 18.38 & -1.59 & 13.62 \\
Bond & -0.16 & -0.71 & 6.07 & -31.44 & 19.52 & -0.23 & 6.52 \\
Credit Spread & 0.34 & -0.36 & 6.41 & -20.75 & 40.06 & 1.41 & 9.53 \\
S\&P 500 & 0.83 & 1.35 & 4.29 & -16.80 & 10.93 & -0.70 & 4.17 \\
Size Spread & 0.05 & -0.01 & 3.29 & -16.38 & 18.41 & 0.26 & 7.89 \\
Emerging Mkts & 0.67 & 0.85 & 6.71 & -28.91 & 17.14 & -0.70 & 4.97 \\
PTFSBD & -1.33 & -3.70 & 15.31 & -26.63 & 68.86 & 1.34 & 5.37 \\
PTFSFX & -0.61 & -4.86 & 19.58 & -30.13 & 90.27 & 1.38 & 5.60 \\
PTFSCOM & -0.28 & -2.96 & 14.28 & -24.65 & 64.75 & 1.09 & 4.65 \\
PTFSIR & -0.66 & -5.75 & 25.84 & -35.13 & 221.92 & 4.28 & 30.75 \\
PTFSSTK & -4.99 & -6.89 & 13.38 & -30.19 & 60.48 & 1.35 & 6.46 \\
ATM PUT & -16.74 & -49.48 & 86.39 & -96.63 & 333.20 & 1.43 & 4.78 \\
OTM PUT & -18.62 & -56.83 & 90.00 & -97.09 & 345.90 & 1.54 & 4.98 \\
ATM CALL & -6.15 & -27.32 & 82.55 & -99.55 & 241.70 & 0.70 & 2.44 \\
OTM CALL & -7.45 & -32.38 & 87.23 & -99.50 & 300.00 & 0.86 & 2.93 \\
\hline
\end{tabular}

This table features summary statistics associated with the benchmark risk factors that we use to evaluate the performance of hedge funds. The acronyms MKT, SMB, HML and MOM in the first column refer respectively to the CRSP value-weighted portfolio, the Small minus Big factor, the High minus Low factor and the Momentum factor obtained from Kenneth French online Data Library. The Bond Market factor and Credit Spread factor are constructed using the monthly change in the 10-Year constant maturity yield and Moody's Baa yield minus the 10-Year constant maturity yield following Fung and Hsieh (2001) (available at the H15 forms, Federal Board of Governors and at St. Louis FRED respectively). The S\&P 500 return is constructed using the total returns from Datastream (RI code) as in Fung and Hsieh (2001). The size spread is constructed as in Fung and Hsieh (2001) using the difference between the Russel 2000 and the S\&P 500 total return from Datastream. The emerging market factor is constructed using the MSCI Emerging Market index monthly total return from Datastream. The trend following strategies (PTFS) are available in Fung's web site. The four option strategies are constructed following Agarwal and Naik (2004) using raw data from Option Metrics. From left to right we report the mean, median, standard deviation, minimum, maximum, skewness and kurtosis statistics for each factor. 
Table 6: Agarwal and Naik (2004) Lambda Estimation

\begin{tabular}{|c|c|c|c|c|c|c|c|}
\hline Gamma & -3.5 & -2 & -1 & -0.5 & 0 & 0.5 & 1 \\
\hline & & & & & & & \\
\hline Asym.) & & & & & & & \\
\hline T-Stat (Boots & $(-2.2)$ & $(-1.74)$ & $(-1.55)$ & $(-1.43)$ & $(-1.26)$ & $(-1.12)$ & $(-1.09)$ \\
\hline Pricing Error & {$[0.0]$} & {$[0]$} & {$[0]$} & {$[0]$} & {$[0]$} & {$[0.0]$} & {$[0.0]$} \\
\hline OTM PUT & 1.29 & 1.76 & 2.10 & 2.26 & 2.27 & 2.08 & 1.76 \\
\hline T-Stat (Asym.) & $(3.93)$ & & & $(2.17)$ & & & (1.64) \\
\hline T-St & $(2.52)$ & (1.94) & $(1.82)$ & (1.68) & $(1.54)$ & $(1.39)$ & $(1.42)$ \\
\hline Pricing Error & {$[0.0]$} & {$[0]$} & {$[0]$} & {$[0]$} & {$[0]$} & {$[0.0]$} & {$[0.0]$} \\
\hline & -0.62 & -0.80 & -0.82 & -0.73 & -0.49 & & \\
\hline Stat (Asym.) & $(-1.01)$ & $(-0.97)$ & $(-0.73)$ & $(-0.55)$ & $(-0.35)$ & $(-0.1)$ & $(0.15)$ \\
\hline & $-0.76)$ & $(-0.7)$ & $(-0.5)$ & $(-0.41)$ & $(-0.25)$ & $(-0.0$ & $(0.12)$ \\
\hline Prici & {$[0]$} & {$[0]$} & {$[0]$} & {$[0]$} & {$[0]$} & {$[0.0]$} & {$[0.0]$} \\
\hline OTI & 0.74 & 1.02 & 1.18 & 1.17 & 1.00 & 0. & 0.35 \\
\hline & $(1.37)$ & $(1.3$ & $(1.16)$ & $(0.98)$ & $(0.77)$ & $(0$. & $(0.32)$ \\
\hline $\mathrm{T}-\mathrm{St}$ & (1.01) & $(0.99)$ & $(0.8)$ & $(0.72)$ & $(0.56)$ & $(0$. & $(0.25)$ \\
\hline Pricing & {$[0]$} & {$[0]$} & {$[0]$} & {$[0]$} & {$[0]$} & {$[0$} & {$[0.1]$} \\
\hline Bond & -0.71 & -0.76 & -0.59 & -0.38 & -0.18 & & 0.09 \\
\hline & & & & & & & \\
\hline & $(-0.87)$ & $(-0.64)$ & $(-0.41)$ & $(-0.25)$ & $(-0.12)$ & $(-0.01)$ & $(0.08)$ \\
\hline Pric & {$[0]$} & {$[0]$} & {$[0]$} & {$[0]$} & {$[0]$} & {$[0.0]$} & {$[0.0]$} \\
\hline & -1.35 & -1.80 & -2.38 & -2.67 & -2.81 & -2 . & -2.30 \\
\hline & $(-1.65)$ & $(-1.48)$ & $(-1.83)$ & $(-2)$ & $(-2.06)$ & $(-1$. & $(-2.08)$ \\
\hline & & $(-1.42)$ & $(-1.54)$ & $(-1.66)$ & $(-1.6)$ & $(-1.8)$ & $(-1.71)$ \\
\hline Pricing Error & {$[0]$} & {$[0]$} & {$[0]$} & {$[0]$} & {$[0]$} & {$[0.0]$} & {$[0.0]$} \\
\hline & & & -9.10 & & -11.11 & -10 & \\
\hline & $(-6.98)$ & $(-4.02)$ & $(-3.74)$ & $(-3.53)$ & $(-3.37)$ & $(-3.36)$ & $(-3.64)$ \\
\hline $\mathrm{T}-\mathrm{St}$ & $(-2.87)$ & $(-2.94)$ & $(-3.05)$ & $(-2.92)$ & $(-2.74)$ & $(-3.2)$ & $(-3.34)$ \\
\hline Prici & {$[0]$} & {$[0]$} & {$[0]$} & {$[0]$} & {$[0]$} & {$[0]$} & {$[0]$} \\
\hline Size & -0.75 & -0.71 & -0.23 & -0.04 & -0.01 & 0.01 & 0.05 \\
\hline & $(-0.6)$ & & & $(-0.02)$ & $(0)$ & & \\
\hline $\mathrm{T}-\mathrm{St}$ & $(-0.54)$ & $(-0.36)$ & $(-0.1)$ & $(-0.02)$ & (0) & $(0)$ & $(0.03)$ \\
\hline Pricing Error & {$[0]$} & {$[0]$} & {$[0]$} & {$[0]$} & {$[0]$} & {$[0.0]$} & {$[0.0]$} \\
\hline Emerging & 0.91 & 1.34 & 1.73 & 1.88 & 1.84 & 1.63 & 1.32 \\
\hline & $(1.85)$ & (1.66) & (1.37) & $(1.2)$ & $(1.1)$ & & $(1.01)$ \\
\hline T-Stat (Boots) & $(1)$ & $(1.03)$ & $(0.98)$ & $(0.99)$ & $(0.95)$ & $(0.99)$ & $(0.99)$ \\
\hline Pricing Error & {$[0]$} & {$[0]$} & {$[0]$} & {$[0]$} & {$[0]$} & {$[0.0]$} & {$[0.0]$} \\
\hline
\end{tabular}

This table presents the estimated Lagrange multiplier $\lambda$ associated with the Cressie Read stochastic discount factor when we take the CRSP value weighted market returns as the only benchmark asset. Columns indicate the Cressie Read $\gamma$ used in the estimation. We report the estimated $\lambda$, the associated $t$-statistics (in parenthesis) as well as the pricing errors from the estimation in basis points (in brackets). T statistics are calculated using both asymptotic distribution (Asym.) as well as a non-parametric pairs bootstrap with 1000 re-samples (Boots). The estimation is based on the full sample from January 1994 to June 2015. 
Table 7: Fung and Hsieh (2001) Alphas for Hedge Fund Indexes

\begin{tabular}{|c|c|c|c|c|c|c|c|c|}
\hline & -3.5 & -2 & -1 & -0.5 & 0 & 0.5 & 1 & Lin \\
\hline Convertible Arbitrage & 0.31 & 0.31 & 0.31 & 0.30 & 0.30 & 0.29 & 0.29 & 0.29 \\
\hline Stud. t (asymp.) & $(2.77)$ & $(2.53)$ & $(2.90)$ & $(3.06)$ & $(3.28)$ & $(3.54)$ & $(3.83)$ & $(1.74)$ \\
\hline Stud. t (boot.) & $(2.37)$ & $(2.55)$ & $(2.61)$ & $(2.64)$ & $(2.49)$ & $(2.4)$ & $(2.36)$ & $(1.74)$ \\
\hline CTA & 0.70 & 0.67 & 0.63 & 0.61 & 0.60 & 0.59 & 0.58 & 0.58 \\
\hline Stud. t (asymp.) & $(3.94)$ & $(3.96)$ & $(5.31)$ & $(6.76)$ & $(8.67)$ & $(10.39)$ & $(11.39)$ & $(4.81)$ \\
\hline Stud. t (boot.) & $(5.33)$ & $(5.41)$ & $(5.54)$ & $(5.36)$ & $(5.19)$ & $(5.21)$ & $(5.04)$ & $(4.85)$ \\
\hline Emerging Markets & 0.07 & 0.16 & 0.22 & 0.26 & 0.30 & 0.35 & 0.38 & 0.38 \\
\hline Stud. t (asymp.) & $(0.67)$ & $(0.54)$ & $(0.78)$ & $(0.98)$ & $(1.31)$ & $(1.69)$ & $(2.02)$ & $(2.28)$ \\
\hline Stud. t (boot.) & $(0.32)$ & $(0.8)$ & $(1.24)$ & $(1.49)$ & $(1.86)$ & $(2.3)$ & $(2.56)$ & $(2.26)$ \\
\hline Equity Market Neutral & 0.35 & 0.35 & 0.35 & 0.34 & 0.34 & 0.34 & 0.35 & 0.35 \\
\hline Stud. t (asymp.) & $(9.15)$ & $(10.13)$ & $(14.63)$ & $(15.96)$ & $(16.22)$ & $(16.18)$ & $(16.56)$ & $(5.69)$ \\
\hline Stud. t (boot.) & $(4.9)$ & $(5.46)$ & $(5.72)$ & $(6.14)$ & $(6.39)$ & $(6.11)$ & $(6.69)$ & $(5.58)$ \\
\hline Event Driven & 0.33 & 0.35 & 0.37 & 0.37 & 0.39 & 0.40 & 0.41 & 0.41 \\
\hline Stud. t (asymp.) & $(2.43)$ & $(2.12)$ & $(2.68)$ & $(3.11)$ & $(3.70)$ & $(4.27)$ & $(4.76)$ & $(5.06)$ \\
\hline Stud. t (boot.) & $(3.79)$ & $(4.47)$ & $(4.85)$ & $(5.25)$ & $(5.68)$ & $(5.96)$ & $(6.38)$ & $(5.15)$ \\
\hline Fixed Income Arbitrage & 0.44 & 0.45 & 0.43 & 0.42 & 0.42 & 0.41 & 0.42 & 0.42 \\
\hline Stud. t (asymp.) & $(7.33)$ & $(7.51)$ & $(8.86)$ & $(8.98)$ & $(8.77)$ & $(8.74)$ & $(8.97)$ & $(4.39)$ \\
\hline Stud. t (boot.) & $(4.92)$ & $(5.39)$ & $(5.46)$ & $(5.58)$ & $(5.22)$ & $(5.29)$ & $(5.54)$ & $(4.64)$ \\
\hline Fund of Funds & 0.20 & 0.21 & 0.20 & 0.20 & 0.20 & 0.21 & 0.21 & 0.21 \\
\hline Stud. t (asymp.) & $(2.40)$ & $(2.30)$ & $(2.74)$ & $(3.04)$ & $(3.47)$ & $(3.89)$ & $(4.27)$ & $(3.03)$ \\
\hline Stud. t (boot.) & $(2.62)$ & $(2.93)$ & $(2.83)$ & $(2.94)$ & $(2.95)$ & $(3.14)$ & $(3.18)$ & $(2.95)$ \\
\hline Global Macro & 0.43 & 0.42 & 0.43 & 0.44 & 0.45 & 0.45 & 0.45 & 0.45 \\
\hline Stud. t (asymp.) & $(5.71)$ & $(6.07)$ & $(7.54)$ & $(7.95)$ & $(8.14)$ & $(8.37)$ & $(8.81)$ & $(4.97)$ \\
\hline Stud. t (boot.) & $(3.74)$ & $(3.9)$ & $(4.47)$ & $(4.6)$ & $(4.88)$ & $(4.89)$ & $(4.79)$ & $(4.71)$ \\
\hline Long/Short Equity Hedge & 0.42 & 0.44 & 0.43 & 0.42 & 0.42 & 0.42 & 0.42 & 0.42 \\
\hline Stud. t (asymp.) & $(2.25)$ & $(1.92)$ & $(2.26)$ & $(2.46)$ & $(2.73)$ & $(2.99)$ & $(3.22)$ & $(4.58)$ \\
\hline Stud. t (boot.) & $(4.8)$ & $(5.48)$ & $(5.72)$ & $(5.62)$ & $(5.65)$ & $(5.51)$ & $(5.53)$ & $(4.57)$ \\
\hline Managed Futures & 0.93 & 0.84 & 0.76 & 0.73 & 0.71 & 0.69 & 0.69 & 0.69 \\
\hline Stud. t (asymp.) & $(3.07)$ & $(3.16)$ & $(4.31)$ & $(5.63)$ & $(7.39)$ & $(8.98)$ & $(10.14)$ & $(3.91)$ \\
\hline Stud. t (boot.) & $(3.94)$ & $(3.83)$ & $(3.81)$ & $(3.84)$ & $(3.7)$ & $(3.88)$ & $(3.83)$ & $(3.68)$ \\
\hline Multi-Strategy & 0.43 & 0.43 & 0.42 & 0.41 & 0.41 & 0.41 & 0.41 & 0.41 \\
\hline Stud. t (asymp.) & $(4.29)$ & $(4.34)$ & $(5.16)$ & $(5.51)$ & $(5.94)$ & $(6.30)$ & $(6.61)$ & $(5.85)$ \\
\hline Stud. t (boot.) & $(7.09)$ & $(7.69)$ & $(7.54)$ & $(7.79)$ & $(7.52)$ & $(7.26)$ & $(7.35)$ & $(5.95)$ \\
\hline
\end{tabular}

This table presents the estimated non-parametric $\alpha$ measure for Hedge Fund indexes associated with the Cressie Read stochastic discount factor when we use the Fung and Hsieh (2001) factors as benchmark assets. In the last column we also add the OLS Jensen's alpha for comparison. For several values of the Cressie Read $\gamma$ used in the estimation, we report the estimated $\alpha$, the asymptotic Student $t$-statistics as well as the bootstrapped Student t-statistics (calculated using bootstrapped standard errors from 1000 re-samples). Bootstrapped t-statistics for the linear model are obtained with the residual bootstrap method suggested by Kosowski et al. (2006). Asymptotic t-statistics are calculated using the delta method (see section 4.4) to account for the dependence of $\alpha$ on $\lambda$ and the error associated with the estimation of the latter. Hedge Fund indexes are built based on the primary strategy reported by each hedge fund by equally weighting their monthly returns using all available data, after applying the filters described in the online Appendix. The resulting estimation uses the full sample from January 1994 to June 2015. 
Table 8: CAPM Nonlinear Alphas for Hedge Fund Indexes

\begin{tabular}{|c|c|c|c|c|c|c|c|}
\hline & CAPM & $(2)$ & $(3)$ & $(4)$ & $\gamma=-3.5$ & $\gamma=-1$ & $\gamma=0.5$ \\
\hline Convertible Arbitrage & 0.21 & 0.44 & 0.31 & 0.08 & 0.05 & 0.19 & 0.21 \\
\hline Stud. t (NW) & $(1.16)$ & $(2.61)$ & $(2.22)$ & $(0.54)$ & $(0.13)$ & $(0.72)$ & $(0.84)$ \\
\hline Stud. t (boot.) & $(1.17)$ & $(2.5)$ & $(2.19)$ & $(0.55)$ & $(0.25)$ & $(1.32)$ & $(1.57)$ \\
\hline CTA & 0.43 & 0.16 & 0.22 & 0.03 & 0.49 & 0.45 & 0.43 \\
\hline Stud. t (NW) & $(3.58)$ & $(1.08)$ & $(1.5)$ & $(0.15)$ & $(3.26)$ & $(3.38)$ & $(3.34)$ \\
\hline Stud. t (boot.) & $(3.51)$ & $(1.08)$ & $(1.46)$ & $(0.16)$ & $(3.57)$ & $(3.35)$ & $(3.38)$ \\
\hline Emerging Markets & 0.2 & 0.66 & 0.55 & 0.42 & 0.09 & 0.17 & 0.19 \\
\hline Stud. t (NW) & $(0.68)$ & $(2.4)$ & $(1.99)$ & $(1.43)$ & $(0.17)$ & $(0.4)$ & $(0.48)$ \\
\hline Stud. t (boot.) & $(0.7)$ & $(2.38)$ & $(2.04)$ & $(1.41)$ & $(0.33)$ & $(0.79)$ & $(0.94)$ \\
\hline Equity Market Neutral & 0.34 & 0.38 & 0.38 & 0.36 & 0.34 & 0.34 & 0.34 \\
\hline Stud. t (NW) & $(5.92)$ & $(6.16)$ & $(6.22)$ & $(6.29)$ & $(4.71)$ & $(4.94)$ & $(5.02)$ \\
\hline Stud. t (boot.) & $(6.09)$ & $(6.26)$ & $(6.73)$ & $(5.96)$ & $(6.9)$ & $(6.69)$ & $(6.79)$ \\
\hline Event Driven & 0.37 & 0.57 & 0.53 & 0.46 & 0.31 & 0.36 & 0.37 \\
\hline Stud. t (NW) & $(3.37)$ & $(6.03)$ & $(5.56)$ & $(4.37)$ & $(1.2)$ & $(1.85)$ & $(2.02)$ \\
\hline Stud. t (boot.) & $(3.5)$ & $(6.02)$ & $(5.82)$ & $(4.42)$ & $(3.01)$ & $(4.36)$ & $(4.66)$ \\
\hline Fixed Income Arbitrage & 0.33 & 0.52 & 0.47 & 0.34 & 0.23 & 0.32 & 0.33 \\
\hline Stud. t (NW) & $(2.78)$ & $(4.18)$ & $(4.53)$ & $(2.92)$ & $(1.08)$ & $(2.19)$ & $(2.39)$ \\
\hline Stud. t (boot.) & $(2.96)$ & $(4.3)$ & $(4.5)$ & $(3.08)$ & $(1.74)$ & $(3.46)$ & $(3.75)$ \\
\hline Fund of Funds & 0.11 & 0.23 & 0.22 & 0.08 & 0.07 & 0.1 & 0.11 \\
\hline Stud. t (NW) & $(1.13)$ & $(2.68)$ & $(2.56)$ & $(0.84)$ & $(0.36)$ & $(0.66)$ & $(0.73)$ \\
\hline Stud. t (boot.) & $(1.19)$ & $(2.66)$ & $(2.47)$ & $(0.8)$ & $(0.75)$ & $(1.22)$ & $(1.36)$ \\
\hline Global Macro & 0.35 & 0.22 & 0.26 & 0.15 & 0.39 & 0.36 & 0.35 \\
\hline Stud. t (NW) & $(3.36)$ & $(1.89)$ & $(2.29)$ & $(1.18)$ & $(3.53)$ & $(3.25)$ & $(3.2)$ \\
\hline Stud. t (boot.) & $(3.32)$ & $(1.95)$ & $(2.24)$ & $(1.2)$ & $(3.79)$ & $(3.46)$ & $(3.59)$ \\
\hline Long/Short Equity Hedge & 0.38 & 0.4 & 0.41 & 0.3 & 0.37 & 0.38 & 0.38 \\
\hline Stud. t (NW) & $(3.66)$ & $(3.76)$ & $(3.81)$ & $(2.68)$ & $(1.28)$ & $(1.6)$ & $(1.67)$ \\
\hline Stud. t (boot.) & $(3.76)$ & $(3.95)$ & $(3.69)$ & $(2.57)$ & $(4.09)$ & $(4.31)$ & $(4.51)$ \\
\hline Managed Futures & 0.45 & 0.03 & 0.16 & -0.18 & 0.55 & 0.48 & 0.46 \\
\hline Stud. t (NW) & $(2.73)$ & $(0.14)$ & $(0.82)$ & $(-0.79)$ & $(2.67)$ & $(2.68)$ & $(2.61)$ \\
\hline Stud. t (boot.) & $(2.67)$ & $(0.14)$ & $(0.76)$ & $(-0.74)$ & $(2.78)$ & $(2.53)$ & $(2.53)$ \\
\hline Multi-Strategy & 0.36 & 0.41 & 0.42 & 0.33 & 0.35 & 0.36 & 0.36 \\
\hline Stud. t (NW) & $(4.32)$ & $(4.93)$ & $(5.06)$ & $(3.89)$ & $(2.15)$ & $(2.66)$ & $(2.78)$ \\
\hline Stud. t (boot.) & $(4.78)$ & $(5.05)$ & $(5.06)$ & $(3.83)$ & $(5.34)$ & $(5.83)$ & $(6.29)$ \\
\hline
\end{tabular}

This table presents the estimated OLS Jensen's $\alpha$ measure for Hedge Fund indexes when we artificially add higher-order moments of the CRSP value weighted market portfolio in the linear regression framework. For completeness we also include the same estimates for selected values of $\gamma$. In the first column, we include the CAPM alpha to which we add, in the subsequent columns, one at a time, polynomial terms of increasing order. We report the estimated $\alpha$, Newey-West and bootstrapped t-statistics. For the linear model we follow the residual bootstrap suggested by Kosowski et al. (2006). NW t-statistics are calculated using Newey and West (1987) heteroskedasticity and autocorrelation consistent standard errors with the optimal number of lags given by $\left\lfloor 4(T / 100)^{2 / 9}\right\rfloor$. Bootstrap Student t-statistics are calculated using bootstrapped standard errors from 1000 re-samples with a nonparametric pairs bootstrap method. Hedge Fund indexes are built based on the primary strategy reported by each hedge fund by equally weighting their monthly returns using all available data, after applying the filters describe $\$ 8$ n the online Appendix. The resulting estimation uses the full sample from January 1994 to June 2015. 
Table 9: Summary Statistics - Individual Fund Alphas

\begin{tabular}{lcccccccc}
\hline & -3.5 & -2 & -1 & -0.5 & 0 & 0.5 & 1 & Lin \\
\hline Mean & 0.21 & 0.21 & 0.21 & 0.21 & 0.21 & 0.22 & 0.22 & 0.22 \\
Variance & 0.63 & 0.59 & 0.57 & 0.56 & 0.55 & 0.54 & 0.53 & 0.53 \\
Skewness & -0.32 & -0.33 & -0.31 & -0.31 & -0.26 & -0.20 & -0.22 & -0.22 \\
Kurtosis & 7.77 & 7.73 & 7.57 & 7.57 & 7.42 & 7.25 & 7.12 & 7.12 \\
Percent & 0.15 & 0.16 & 0.16 & 0.16 & 0.16 & 0.16 & 0.17 & 0.21 \\
\hline & & \multicolumn{7}{c}{ CAPhart } \\
Mean & 0.18 & 0.18 & 0.17 & 0.17 & 0.16 & 0.16 & 0.16 & 0.16 \\
Var & 0.75 & 0.68 & 0.63 & 0.60 & 0.58 & 0.58 & 0.57 & 0.57 \\
Skew & -0.15 & -0.06 & -0.02 & 0.03 & 0.06 & 0.04 & 0.01 & 0.06 \\
Kurt & 8.40 & 8.10 & 7.95 & 7.97 & 7.80 & 7.90 & 8.05 & 7.97 \\
Percentage & 0.13 & 0.13 & 0.13 & 0.13 & 0.13 & 0.13 & 0.13 & 0.17 \\
\hline
\end{tabular}

Fung and Hsieh

\begin{tabular}{lcccccccc}
\hline Mean & 0.23 & 0.24 & 0.24 & 0.25 & 0.25 & 0.25 & 0.26 & 0.26 \\
Variance & 0.87 & 0.88 & 0.85 & 0.83 & 0.75 & 0.71 & 0.69 & 0.70 \\
Skewness & -0.14 & -0.13 & -0.09 & 0.04 & 0.08 & 0.15 & 0.16 & 0.21 \\
Kurtosis & 7.44 & 7.39 & 7.32 & 7.22 & 6.98 & 6.92 & 6.84 & 6.89 \\
Percentage & 0.11 & 0.11 & 0.12 & 0.12 & 0.12 & 0.13 & 0.13 & 0.22 \\
\hline
\end{tabular}

Agarwal and Naik

\begin{tabular}{lllllllll}
\hline Mean & 0.28 & 0.26 & 0.25 & 0.23 & 0.23 & 0.23 & 0.23 & 0.23 \\
Variance & 1.04 & 1.06 & 1.02 & 0.98 & 0.90 & 0.88 & 0.83 & 1.00 \\
Skewness & 0.64 & 0.53 & 0.46 & 0.35 & 0.31 & 0.11 & 0.15 & 0.44 \\
Kurtosis & 8.85 & 8.92 & 8.51 & 8.43 & 8.07 & 7.71 & 7.38 & 9.49 \\
Percentage & 0.13 & 0.12 & 0.12 & 0.13 & 0.13 & 0.13 & 0.13 & 0.20 \\
\hline
\end{tabular}

This table presents summary statistics for both Cressie-Read and Jensen's $\alpha$ s of the individual hedge funds in our sample. Each panel corresponds to a different set of benchmark factors. Percentage represents the percentage of alphas that are positive and statistically significant at the $10 \%$ level. To calculate all statistics we winsorize the sample at the $0.05 \%$ level. 
Table 10: Quantiles - Cross-Sectional Distribution of Individual Fund Alphas

\begin{tabular}{|c|c|c|c|c|c|c|c|}
\hline & -3.5 & -2 & -1 & -0.5 & 0 & 0.5 & 1 \\
\hline \multicolumn{8}{|c|}{ Carhart } \\
\hline $1 \%$ & -2.80 & -2.56 & -2.46 & -2.41 & -2.38 & -2.36 & -2.38 \\
\hline $10 \%$ & -0.65 & -0.63 & -0.60 & -0.59 & -0.59 & -0.60 & -0.60 \\
\hline $50 \%$ & 0.18 & 0.17 & 0.16 & 0.16 & 0.16 & 0.16 & 0.15 \\
\hline $90 \%$ & 1.03 & 1.00 & 0.96 & 0.94 & 0.93 & 0.93 & 0.92 \\
\hline $99 \%$ & 2.94 & 2.87 & 2.68 & 2.57 & 2.48 & 2.44 & 2.44 \\
\hline \multicolumn{8}{|c|}{ Agarwal and Naik } \\
\hline $1 \%$ & -2.80 & -2.92 & -2.96 & -3.01 & -2.71 & -2.71 & -2.57 \\
\hline $10 \%$ & -0.66 & -0.70 & -0.68 & -0.70 & -0.66 & -0.64 & -0.63 \\
\hline $50 \%$ & 0.24 & 0.22 & 0.21 & 0.21 & 0.20 & 0.20 & 0.20 \\
\hline $90 \%$ & 1.24 & 1.26 & 1.20 & 1.17 & 1.16 & 1.15 & 1.15 \\
\hline $99 \%$ & 4.09 & 3.90 & 3.96 & 3.69 & 3.42 & 3.31 & 3.17 \\
\hline
\end{tabular}

This table presents five quantiles $(1 \%, 10 \%, 50 \%, 90 \%$ and $99 \%$ ) for each Cressie-Read cross-sectional distribution of individual hedge funds $\alpha \mathrm{s}$ in our sample. Each panel corresponds to a different set of benchmark factors. 
Table 11: Cressie-Read Alphas per Category of Hedge Funds

\begin{tabular}{lccccccc}
\hline & -3.5 & -2 & -1 & -0.5 & 0 & 0.5 & 1 \\
\hline Convertible Arbitrage & 0.16 & 0.17 & 0.20 & 0.20 & 0.22 & 0.23 & 0.24 \\
$10 \%$ & -0.54 & -0.42 & -0.35 & -0.31 & -0.35 & -0.31 & -0.28 \\
$90 \%$ & 0.76 & 0.76 & 0.70 & 0.70 & 0.71 & 0.75 & 0.85 \\
CTA & 0.39 & 0.38 & 0.38 & 0.40 & 0.41 & 0.42 & 0.41 \\
$10 \%$ & -0.74 & -0.78 & -0.79 & -0.78 & -0.74 & -0.79 & -0.78 \\
$90 \%$ & 1.89 & 1.84 & 1.86 & 1.78 & 1.69 & 1.58 & 1.57 \\
Emerging Markets & 0.11 & 0.14 & 0.13 & 0.18 & 0.18 & 0.19 & 0.21 \\
$10 \%$ & -1.14 & -1.22 & -1.21 & -1.12 & -1.10 & -1.07 & -0.94 \\
$90 \%$ & 1.27 & 1.34 & 1.28 & 1.36 & 1.25 & 1.22 & 1.29 \\
Equity Market Neutral & 0.19 & 0.20 & 0.21 & 0.20 & 0.20 & 0.20 & 0.19 \\
$10 \%$ & -0.64 & -0.56 & -0.54 & -0.55 & -0.54 & -0.55 & -0.58 \\
$90 \%$ & 1.07 & 1.07 & 1.09 & 1.10 & 1.02 & 0.93 & 0.88 \\
Event Driven & 0.38 & 0.39 & 0.38 & 0.39 & 0.39 & 0.39 & 0.39 \\
$10 \%$ & -0.30 & -0.30 & -0.29 & -0.31 & -0.27 & -0.25 & -0.23 \\
$90 \%$ & 1.13 & 1.11 & 1.10 & 1.07 & 1.10 & 1.09 & 1.04 \\
Fixed Income Arbitrage & 0.31 & 0.30 & 0.30 & 0.31 & 0.29 & 0.30 & 0.30 \\
$10 \%$ & -0.48 & -0.38 & -0.31 & -0.30 & -0.28 & -0.25 & -0.27 \\
$90 \%$ & 1.02 & 1.11 & 0.96 & 0.97 & 0.88 & 0.88 & 0.89 \\
Fund of Funds & 0.07 & 0.08 & 0.09 & 0.09 & 0.09 & 0.09 & 0.10 \\
$10 \%$ & -0.49 & -0.46 & -0.44 & -0.44 & -0.43 & -0.41 & -0.42 \\
$90 \%$ & 0.60 & 0.60 & 0.60 & 0.61 & 0.59 & 0.59 & 0.60 \\
Global Macro & 0.26 & 0.23 & 0.22 & 0.21 & 0.24 & 0.27 & 0.29 \\
$10 \%$ & -0.86 & -0.85 & -0.91 & -0.77 & -0.71 & -0.56 & -0.51 \\
$90 \%$ & 1.19 & 1.17 & 1.19 & 1.22 & 1.19 & 1.18 & 1.13 \\
Long/Short Equity Hedge & 0.26 & 0.32 & 0.32 & 0.31 & 0.33 & 0.33 & 0.33 \\
$10 \%$ & -0.78 & -0.79 & -0.76 & -0.72 & -0.67 & -0.67 & -0.67 \\
$90 \%$ & 1.31 & 1.27 & 1.21 & 1.21 & 1.22 & 1.21 & 1.21 \\
Managed Futures & 0.41 & 0.41 & 0.41 & 0.42 & 0.42 & 0.43 & 0.43 \\
$10 \%$ & -0.98 & -0.94 & -0.94 & -0.86 & -0.86 & -0.75 & -0.72 \\
$90 \%$ & 1.79 & 1.71 & 1.59 & 1.60 & 1.61 & 1.60 & 1.62 \\
Multi-Strategy & 0.22 & 0.20 & 0.20 & 0.20 & 0.19 & 0.21 & 0.21 \\
$10 \%$ & -0.68 & -0.65 & -0.70 & -0.64 & -0.61 & -0.56 & -0.54 \\
$90 \%$ & 1.07 & 1.04 & 1.02 & 1.01 & 0.95 & 0.97 & 0.94 \\
\hline & & & & & & & \\
& & & & & & &
\end{tabular}

This table presents the average alpha, as well as the 10th and 90th percentiles of the distribution of alphas for the various hedge fund categories and across various Cressie-Read $\gamma \mathrm{s}$. To obtain the alphas, we use the Fung and Hsieh ten factors as basis assets for the SDF estimation. 
Table 12: Alpha Correlations

\begin{tabular}{|c|c|c|c|c|c|c|c|c|}
\hline \multicolumn{9}{|c|}{ Fung and Hsieh: } \\
\hline$\gamma=-3.5$ & 1.00 & 0.89 & 0.82 & 0.77 & 0.74 & 0.71 & 0.69 & 0.63 \\
\hline$\gamma=-2$ & 0.83 & 1.00 & 0.89 & 0.84 & 0.80 & 0.76 & 0.73 & 0.66 \\
\hline$\gamma=-1$ & 0.81 & 0.99 & 1.00 & 0.92 & 0.86 & 0.81 & 0.78 & 0.71 \\
\hline$\gamma=-0.5$ & 0.79 & 0.98 & 0.99 & 1.00 & 0.90 & 0.85 & 0.82 & 0.75 \\
\hline$\gamma=0$ & 0.78 & 0.98 & 0.99 & 0.99 & 1.00 & 0.92 & 0.87 & 0.79 \\
\hline$\gamma=0.5$ & 0.75 & 0.97 & 0.97 & 0.98 & 0.99 & 1.00 & 0.94 & 0.84 \\
\hline$\gamma=1$ & 0.74 & 0.96 & 0.96 & 0.97 & 0.98 & 1.00 & 1.00 & 0.84 \\
\hline Linear & 0.80 & 0.82 & 0.84 & 0.85 & 0.86 & 0.88 & 0.87 & 1.00 \\
\hline \multicolumn{9}{|c|}{ Agarwal and Naik: } \\
\hline$\gamma=-3.5$ & 1.00 & 0.86 & 0.77 & 0.73 & 0.70 & 0.68 & 0.66 & 0.60 \\
\hline$\gamma=-2$ & 0.95 & 1.00 & 0.87 & 0.81 & 0.77 & 0.74 & 0.72 & 0.65 \\
\hline$\gamma=-1$ & 0.89 & 0.96 & 1.00 & 0.90 & 0.85 & 0.80 & 0.77 & 0.71 \\
\hline$\gamma=-0.5$ & 0.87 & 0.93 & 0.95 & 1.00 & 0.91 & 0.85 & 0.81 & 0.76 \\
\hline$\gamma=0$ & 0.87 & 0.93 & 0.94 & 0.97 & 1.00 & 0.90 & 0.87 & 0.81 \\
\hline$\gamma=0.5$ & 0.84 & 0.89 & 0.92 & 0.94 & 0.97 & 1.00 & 0.94 & 0.85 \\
\hline$\gamma=1$ & 0.83 & 0.88 & 0.89 & 0.93 & 0.96 & 0.99 & 1.00 & 0.86 \\
\hline Linear & 0.73 & 0.77 & 0.82 & 0.85 & 0.88 & 0.92 & 0.91 & 1.00 \\
\hline
\end{tabular}

This table presents regular correlations (in the lower triangular matrix) and rank correlations (in the upper triangular matrix) between the alphas of all funds for all pairs of gamma values. In the upper panel, we report the statistics for the Fung and Hsieh set of factors, while in the lower panel we consider the Agarwal and Naik factors. 
Table 13: Explaining Investors' Divergence about fund performance

\begin{tabular}{lcccc}
\hline & \multicolumn{4}{c}{ Basis Assets: } \\
\hline \multirow{4}{*}{ HF Mean } & 0.01 & -0.05 & -0.08 & -0.07 \\
& $(0.43)$ & $(-3.46)$ & $(-2.88)$ & $(-2.44)$ \\
Co-Skewness & 0.14 & 0.13 & 0.07 & 0.04 \\
& $(3.02)$ & $(3.71)$ & $(2.81)$ & $(1.80)$ \\
Co-Kurtosis & 0.27 & 0.15 & 0.02 & 0.02 \\
Idios. Vol. & $(5.86)$ & $(9.13)$ & $(0.64)$ & $(0.60)$ \\
& 0.19 & 0.33 & 0.34 & 0.38 \\
HF Skewness & $(3.73)$ & $(5.41)$ & $(4.89)$ & $(5.31)$ \\
& -0.10 & -0.07 & -0.02 & -0.00 \\
HF Kurtosis & $(-3.76)$ & $(-2.37)$ & $(-0.78)$ & $(-0.01)$ \\
& -0.08 & -0.06 & -0.03 & -0.02 \\
AR(1) & $(-4.76)$ & $(-2.42)$ & $(-1.21)$ & $(-0.66)$ \\
Size & -0.10 & -0.08 & -0.06 & -0.02 \\
& $(-4.18)$ & $(-3.50)$ & $(-2.42)$ & $(-1.01)$ \\
Age & 0.02 & -0.00 & -0.00 & -0.01 \\
& $(1.53)$ & $(-0.15)$ & $(-0.08)$ & $(-0.50)$ \\
$R^{2}$ & -0.06 & -0.22 & -0.33 & -0.17 \\
& $(-2.95)$ & $(-5.98)$ & $(-22.49)$ & $(-7.67)$ \\
& 0.11 & 0.24 & 0.34 & 0.28 \\
\hline
\end{tabular}

This table presents the linear regression estimates for the following regression: $\operatorname{Div}_{i}=\beta_{0}+$ $\sum_{i=1}^{N} \beta_{i} X_{i}+\epsilon_{i}$, where $X_{i}$ denotes the control variables in the regression (lines in the table above) and Divi is the logarithm of the crosssectional variance of the various Cressie-Read alphas. Columns indicate the set of factors used in the alpha estimation (CAPM stands for the CRSP value weighted market portfolio, FFC for the Carhart four factor model, FH for the Fung and Hsieh ten factor model, and AN for the Agarwal and Naik nine factor model). Although not reported all regressions include dummies for the hedge fund primary category. Student t-statistics are computed using robust standard errors clustered by primary category. 


\section{Weights on Mixed Moments - Alpha Taylor Expansion}
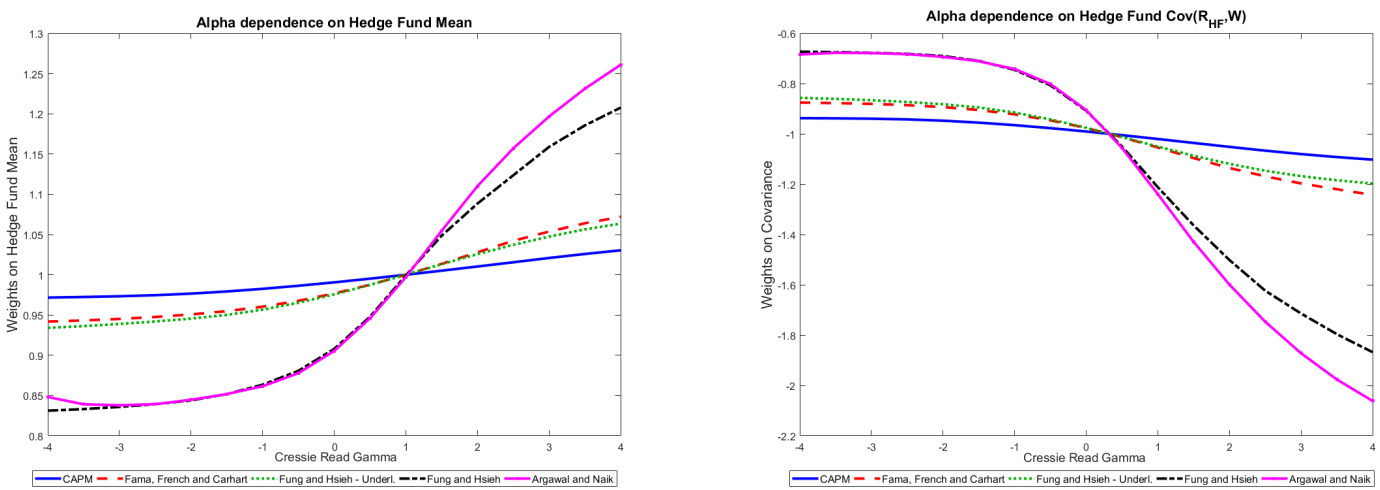

Alpha dependence on $\operatorname{Coskew}\left(R_{H F}, W\right.$
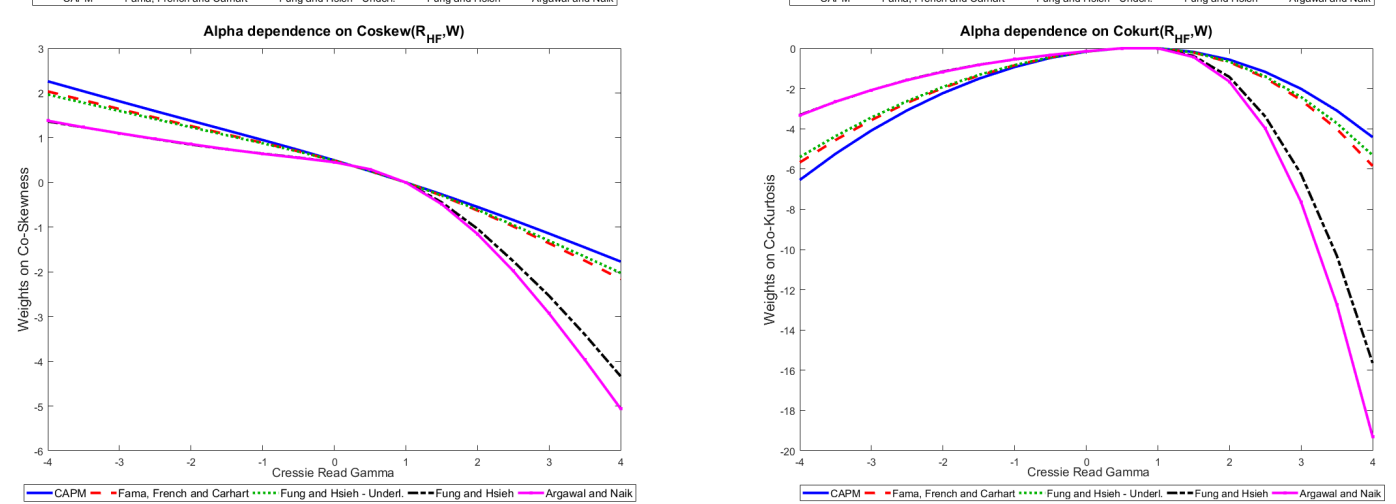

Figure 1: This figure plots the implied weights on the hedge fund average return, the co-variance with the endogenous portfolio, the co-skewness and co-kurtosis based on a Taylor expansion of the SDF alpha. Weights are calculated for five possible combinations of basis assets: the CRSP value weighted market return, the Carhart (1997) factors, the Fung and Hsieh (2001) underlying factors plus the full set of ten factors, and the Agarwal and Naik (2004) factors. The specific factors in each model are defined in section 5.2. Weights are calculated using the optimal $\lambda$ for a grid of $\gamma_{\mathrm{s}}$ between -4 and 4 with 0.5 increments. 


\section{Expected Excess Return of the Optimal Endogenous Cressie-Read Portfolio}

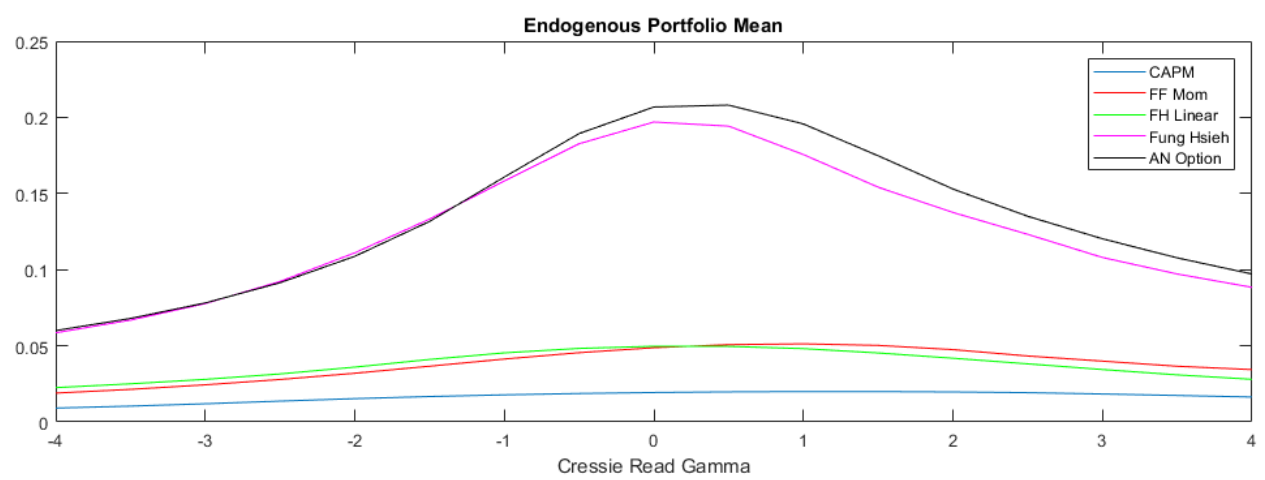

Figure 2: This figure plots the expected excess return $E\left[-\lambda_{\gamma}^{\prime} \tilde{\mathbf{R}}\right]$ of the optimal portfolio solving the optimization problem in Corollary 1 . The expected excess return is estimated for five possible combinations of basis assets: the CRSP value weighted market return, the Carhart (1997) factors, the Fung and Hsieh (2001) underlying factors plus the full set of ten factors, and the Agarwal and Naik (2004) factors. Estimation is based on the optimal $\lambda\left(\hat{\lambda_{\gamma}}\right)$ for a grid of $\gamma_{\mathrm{s}}$ between -4 and 4 with 0.5 increments, and on a risk-free rate $R_{F}=1$.

\section{Implied Risk Neutral Distribution}

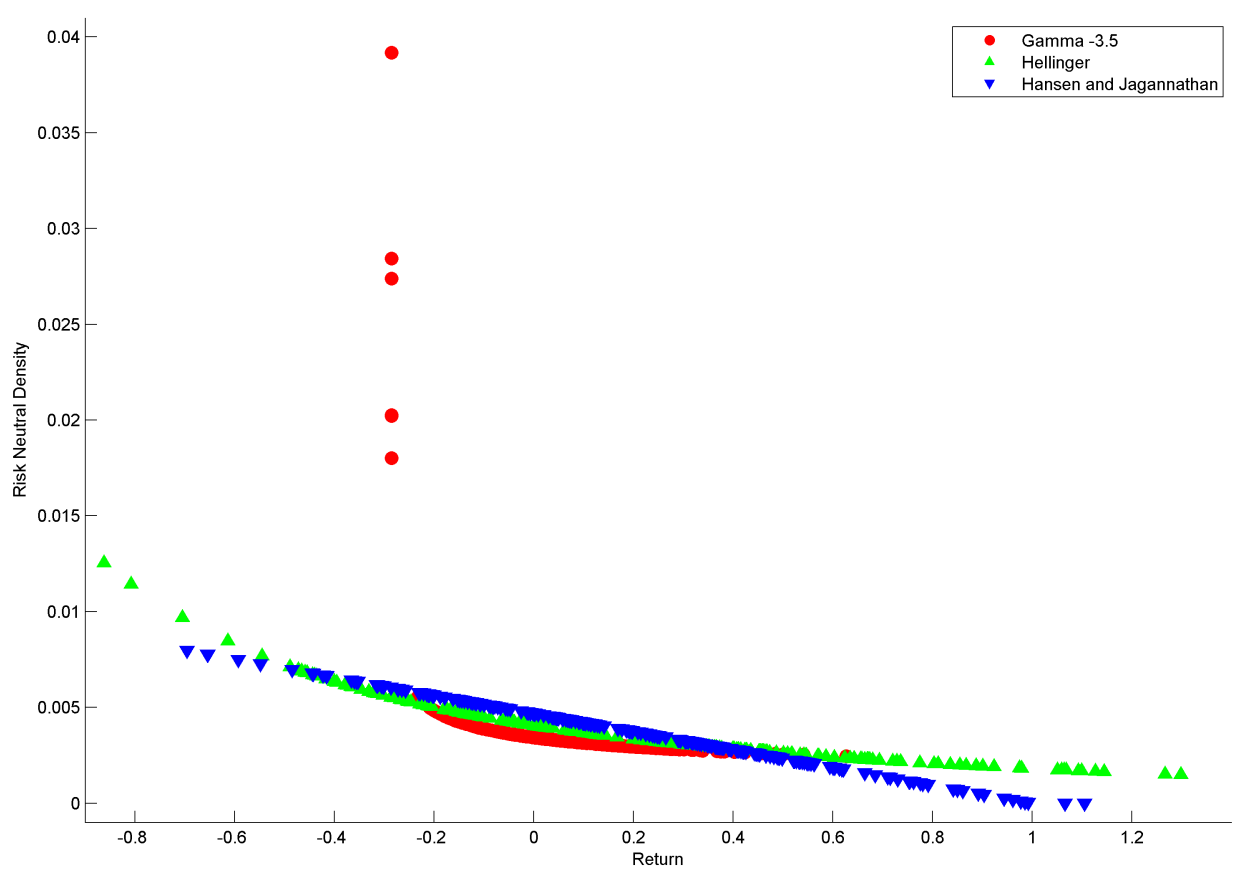

Figure 3: This figure plots the estimated risk neutral distribution for $\gamma=\{-3.5,-0.5,1\}$ against the endogenous portfolio $\left(\lambda(\gamma, R)^{\prime} R\right)$. To estimate the stochastic discount factor we consider the full sample for the Fung and Hsieh (2001) factors and $E[m]=1$. 


\section{Estimated alpha in a one-factor example}

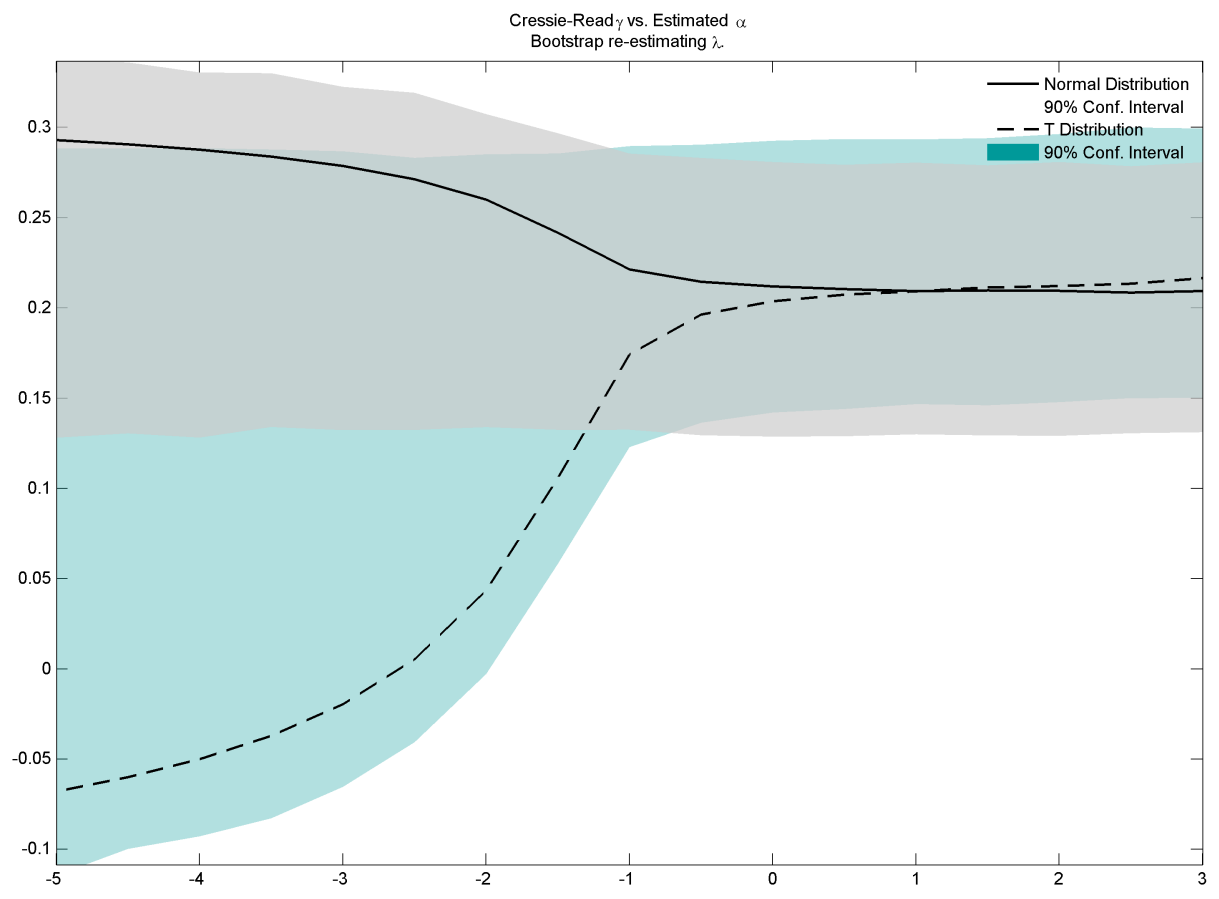

Figure 4: This figure presents the estimated alpha for various values of $\gamma$ when we use a unique asset as basis for the stochastic discount factor estimation. To generate the underlying data we simulate the market returns from a non-central Student t-distribution with a non-centrality parameter equal to 0.2 and a number of degrees of freedom $\nu=6$. The hedge fund returns are simulated from a non-central Student-t distribution with a non-centrality parameter equal to 0.2 and a number of degrees of freedom $\nu=4$ and from a Gaussian distribution that matches the mean and variance of the non-central Student-t distribution. When generating our sample we force the sample mean and variance for both hedge funds to be equal. Confidence intervals are calculated via bootstrap with 10,000 draws. 


\section{Stochastic Discount Factor Time Series}
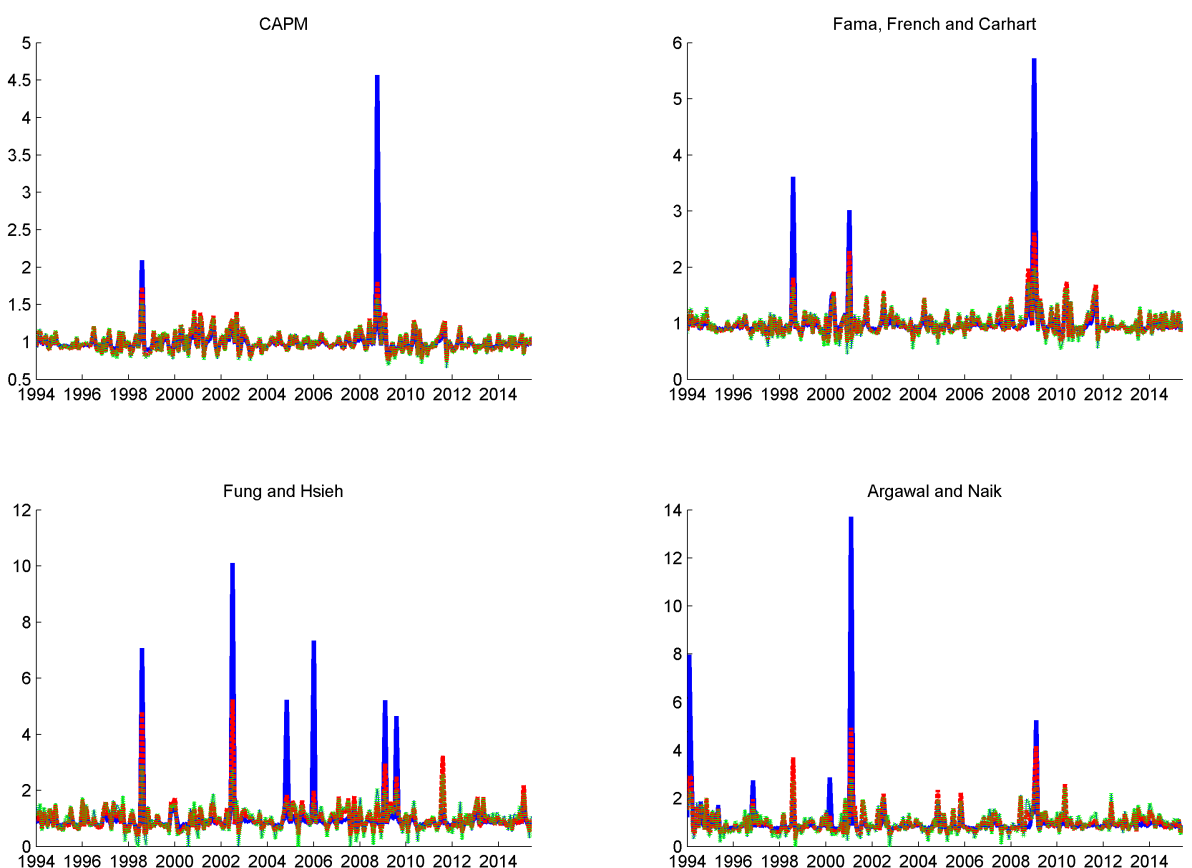

Gamma $=-3.5$

Hansen and Jagannathan

Figure 5: This figure plots the time series of the estimated stochastic discount factors for $\gamma=\{-3.5,-0.5,1\}$ when we consider four sets of basis assets: the CRSP value weighted market return, the Carhart (1997) factors, the Fung and Hsieh (2001) factors, and the Agarwal and Naik (2004) factors. 


\section{Cressie-Read Alpha vs. Jensen's Alpha}
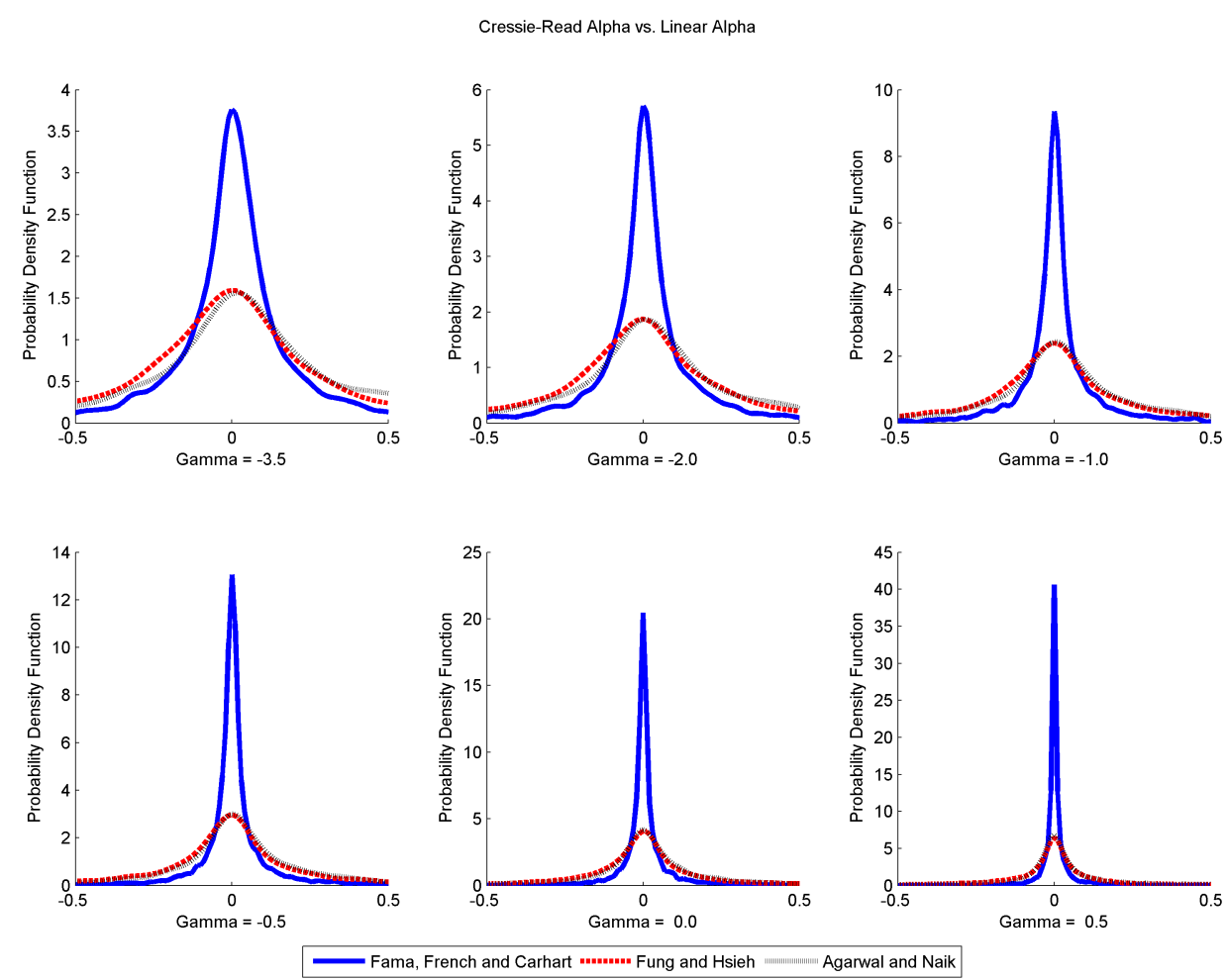

Figure 6: This figure plots the kernel density for the difference between Cressie-Read alphas and Jensen's Alpha (estimate via OLS) for a combination of three sets of basis assets and six values of $\gamma$. The basis assets are, respectively, the Carhart (1997) factors, the Fung and Hsieh (2001) underlying factors plus the full set of ten factors, and the Agarwal and Naik (2004) factors. The CAPM plot is omitted since alpha estimates for most models are similar. The values of $\gamma$ used are indicated below each plot. Alphas are calculated for each hedge fund using an estimated SDF matching the fund time series. 


\section{Cressie-Read Alpha vs. Jensen's Alpha - T-statistics}
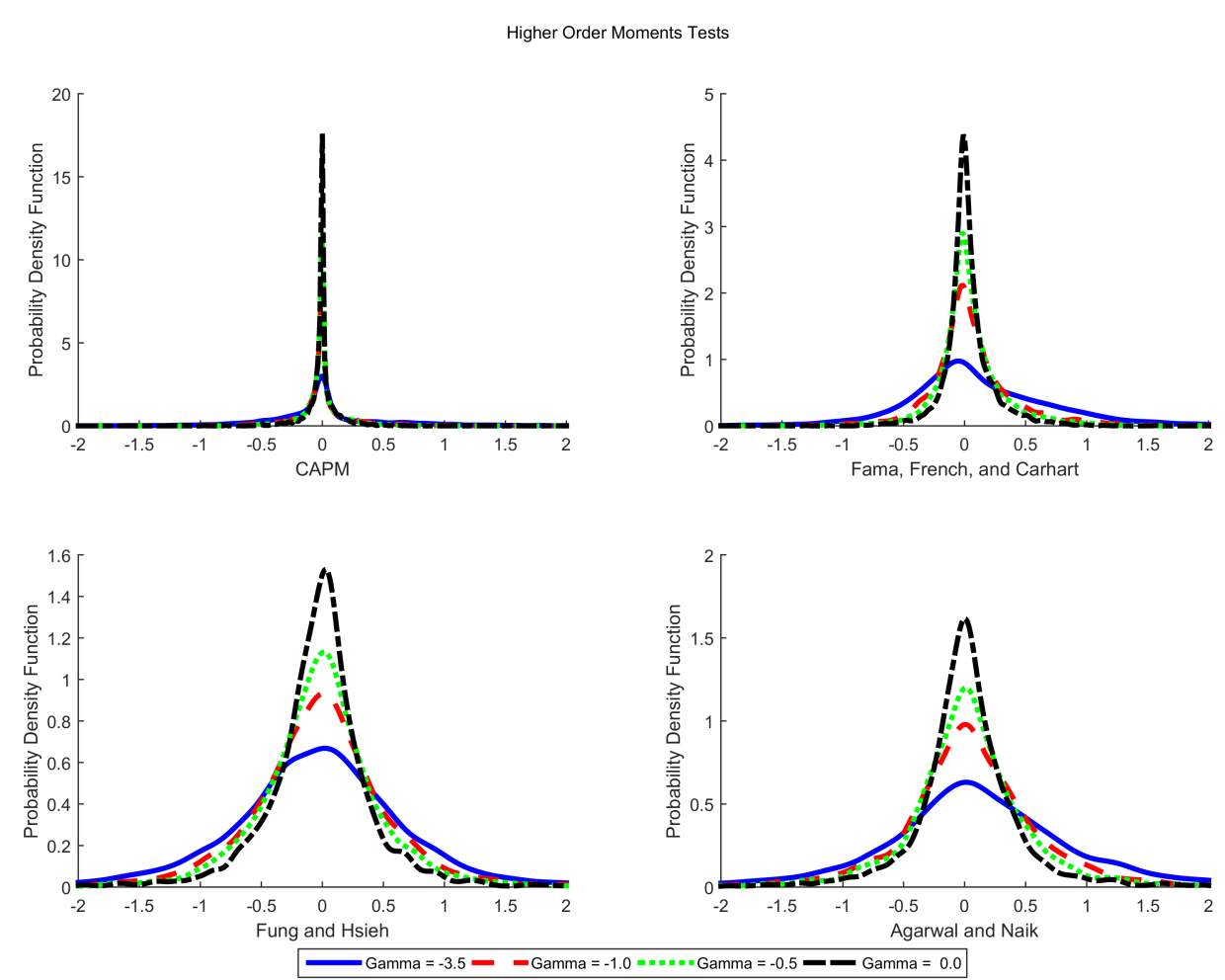

Figure 7: This figure plots the kernel density for the cross-sectional t-statistics for a test of equality between Cressie-Read and Jensen's alpha estimates. Each panel plots the distribution for a given set of basis assets and four values for the Cressie-Read $\gamma$. The basis assets are, respectively, the CRSP value weighted market portfolio (CAPM), the Carhart (1997) factors, the Fung and Hsieh (2001) underlying factors plus the full set of ten factors, and the Agarwal and Naik (2004) factors. The values of $\gamma$ used are indicated below each plot. Alphas are calculated for each hedge fund using an estimated SDF matching the fund time series. The t-statistics are calculated based on a paired test using Newey and West (1987) standard errors. 


\section{T-Statistics Comparison: Asymptotic Distribution}
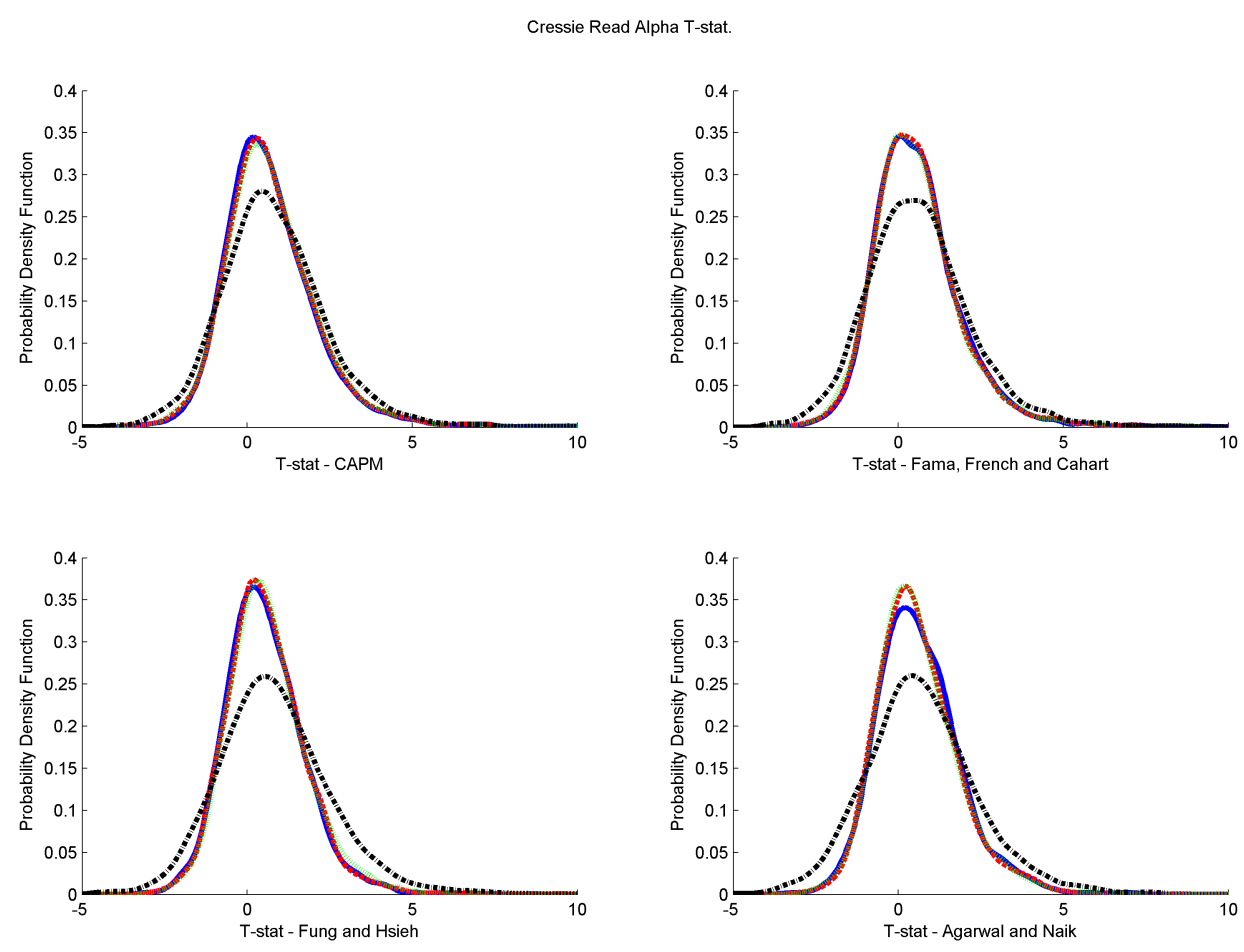

Gamma $=-3.5 \ldots \ldots+. . .6$ Gamma $=-1.0 \quad$ Gamma $=1.0 \ldots \ldots \ldots$ Linear

Figure 8: This figure plots the kernel density for the alpha Student t-statistics for four sets of basis assets: the CRSP value weighted market return, the Carhart (1997) factors, the Fung and Hsieh (2001) underlying factors plus the full set of ten factors, and the Agarwal and Naik (2004) factors. Each plot contains the estimated t-statistics density for four values of gamma $(\gamma=\{-3.5,-1,0,1\})$ and the Jensen's Alpha (estimated via OLS). Alphas and t-statistics are calculated for each hedge fund using an estimated SDF matching the fund time series. 


\section{T-Statistics Comparison: Bootstrap}
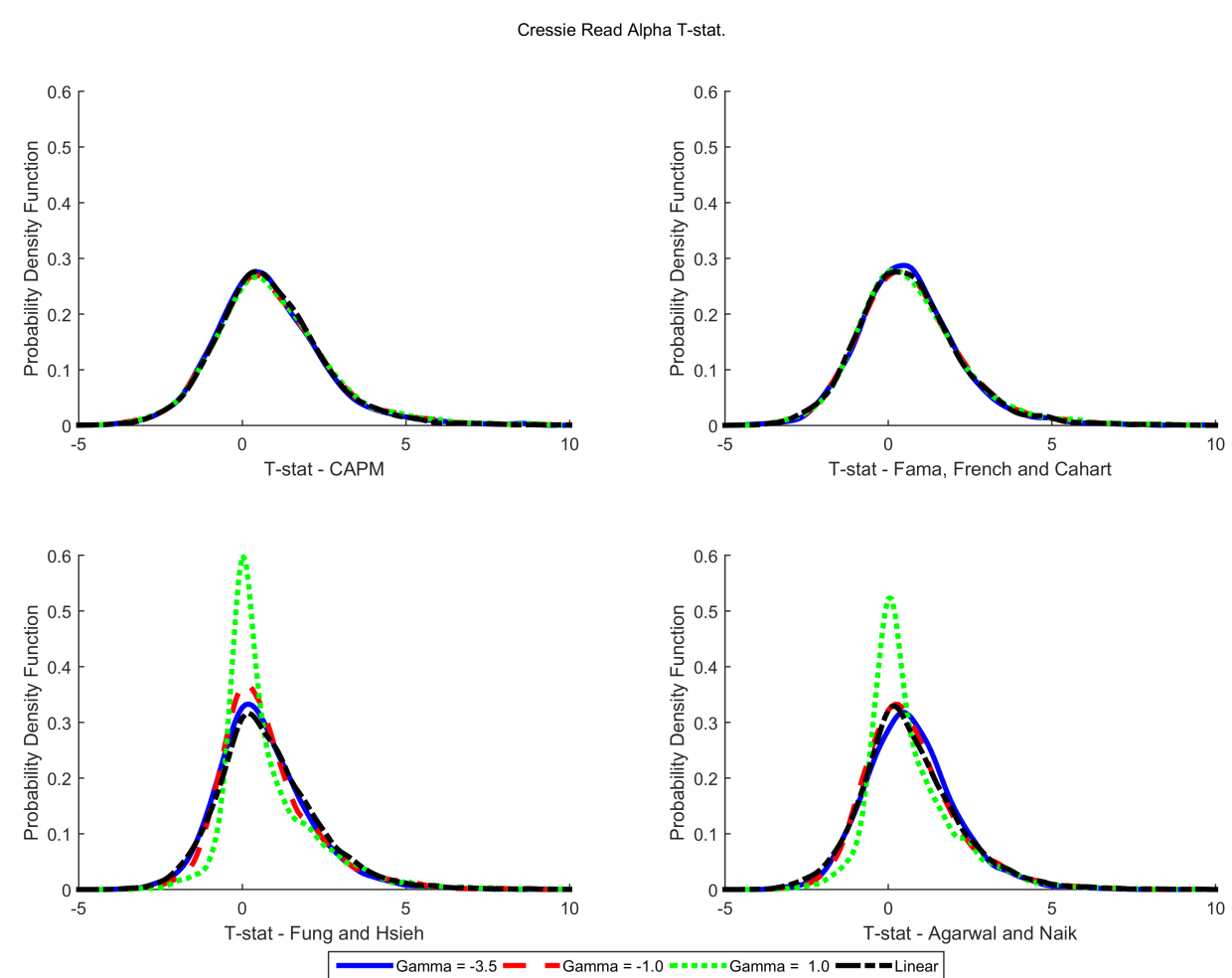

Figure 9: This figure plots the kernel density for the Alpha Bootstrapped t-statistics for four sets of basis assets: the CRSP value weighted market return, the Carhart (1997) factors, the Fung and Hsieh (2001) underlying factors plus the full set of ten factors, and the Agarwal and Naik (2004) factors. Each plot contains the estimated T-statistics density for three values of gamma $(\gamma=\{-3.5,-1,1\})$ and the Jensen's Alpha (estimated via OLS). Cressie-Read bootstrapped t-statistics are based on a non-parametric bootstrap with 1000 re-samples for both hedge fund returns and basis factors. In each bootstrap we re-estimate the SDF $\lambda$ vector. Jensen's Alpha bootstraps are based on the residual bootstrap of Kosowski et al. (2006). 
Ranking Disagreement: Jensen's - Average Cressie-Read $(\gamma \leq 0)$

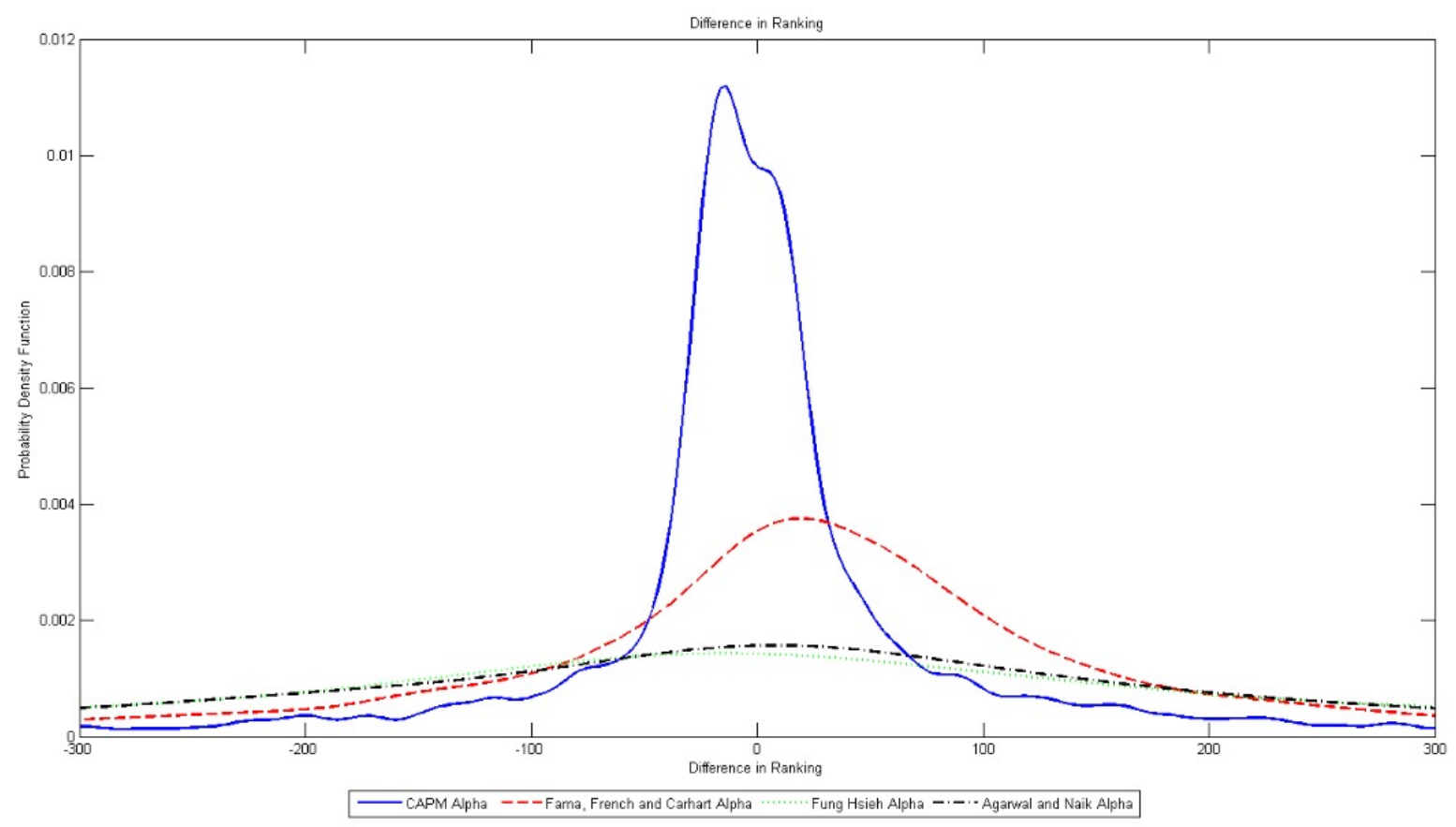

Figure 10: This figure illustrates the ranking differences between the Jensen's alphas and the average of the alpha rankings obtained with the negative values of gamma. The four curves correspond to the four sets of factors considered: CAPM, Carhart, Fung and Hsieh and Agarwal and Naik. 